\title{
Carbon sources in suspended particles and surface sediments from the Beaufort Sea revealed by molecular lipid biomarkers and compound-specific isotope analysis
}

\author{
I. Tolosa ${ }^{1}$, S. Fiorini ${ }^{1, *}$, B. Gasser ${ }^{1}$, J. Martín ${ }^{1,2}$, and J. C. Miquel ${ }^{1}$ \\ ${ }^{1}$ International Atomic Energy Agency - Environment Laboratories, 4, quai Antoine 1er, MC 98000, Monaco \\ ${ }^{2}$ Instituto de Ciencias del Mar, CSIC, Paseo Marítimo de la Barceloneta, 37-49, 08003 Barcelona, Spain \\ * now at: Ecole Normale Supérieure, CEREEP ECOTRON-Ile de France, CNRS-ENS UMS 3194, 78 rue du chateau, \\ 77140, Saint-Pierre les Nemours, France
}

Correspondence to: I. Tolosa (i.Tolosa@iaea.org)

Received: 29 June 2012 - Published in Biogeosciences Discuss.: 12 October 2012

Revised: 31 January 2013 - Accepted: 21 February 2013 - Published: 26 March 2013

\begin{abstract}
Molecular lipid biomarkers (hydrocarbons, alcohols, sterols and fatty acids) and compound-specific isotope analysis of suspended particulate organic matter (SPM) and surface sediments of the Mackenzie Shelf and slope (southeast Beaufort Sea, Arctic Ocean) were studied in summer 2009. The concentrations of the molecular lipid markers, characteristic of known organic matter sources, were grouped and used as proxies to evaluate the relative importance of fresh algal, detrital algal, fossil, $\mathrm{C}_{3}$ terrestrial plants, bacterial and zooplankton material in the organic matter $(\mathrm{OM})$ of this area. Fossil and detrital algal contributions were the major fractions of the freshwater SPM from the Mackenzie River with $\sim 34 \%$ each of the total molecular biomarkers. Fresh algal, $\mathrm{C}_{3}$ terrestrial, bacterial and zooplanktonic components represented much lower percentages, $17,10,4$ and $<1 \%$, respectively. In marine SPM from the Mackenzie slope, the major contributions were fresh and detrital algal components $(>80 \%)$, with a minor contribution of fossil and $\mathrm{C}_{3}$ terrestrial biomarkers. Characterization of the sediments revealed a major sink of refractory algal material mixed with some fresh algal material, fossil hydrocarbons and a small input of $\mathrm{C}_{3}$ terrestrial sources. In particular, the sediments from the shelf and at the mouth of the Amundsen Gulf presented the highest contribution of detrital algal material (60-75\%), whereas those from the slope contained the highest proportion of fossil $(40 \%)$ and $\mathrm{C}_{3}$ terrestrial plant material (10\%). Overall, considering that the detrital algal material is marine derived, autochthonous sources
\end{abstract}

contributed more than allochthonous sources to the OM lipid pool. Using the ratio of an allochthonous biomarker (normalized to total organic carbon, TOC) found in the sediments to those measured at the river mouth water, we estimated that the fraction of terrestrial material preserved in the sediments accounted for $30-40 \%$ of the total carbon in the inner shelf sediments, $17 \%$ in the outer shelf and Amundsen Gulf and up to $25 \%$ in the slope sediments. These estimates are low compared to other studies conducted 5-20 yr earlier, and they support the increase in primary production during the last decade mainly because of the increase in the number of icefree days and due to the strength and persistence of winds favouring upwelling.

\section{Introduction}

The Arctic Ocean is known to be very sensitive to climate change. Some consequences of global warning on the Arctic environment are reduction of the ice cover and thawing of the carbon-rich permafrost. This leads to an increase of the surface exposed to solar radiation and of the input of carbon into the ocean, both favourable to phytoplankton growth (Arrigo et al., 2008; Barber and Hanesiak, 2004; Johannessen et al., 2004). Therefore a better knowledge of this precarious environment is crucial. A major contribution of terrigenous particulate organic carbon (POC) to continental shelves is the discharge from large Arctic rivers which is further amplified 
by the input resulting from coastal erosion (Stein and Macdonald, 2004). The Mackenzie River, with a freshwater discharge of $281 \mathrm{~km}^{3} \mathrm{yr}^{-1}$ into the Beaufort Sea, is the most important in terms of POC contribution to the Arctic Ocean (Rachold et al., 2004) and dominates sediment supply to the Canadian Beaufort Sea (Macdonald et al., 1998; Rachold et al., 2000). Available estimations specify that about $50 \%$ of the $127 \mathrm{Mt}$ of the terrigenous sediments annually provided by the Mackenzie River are rapidly accumulated in the delta front area, $40 \%$ stays on the shelf, and $10 \%$ escapes to the slope and farther (Macdonald et al., 1998). Marine organic carbon from primary and secondary production has been estimated at $\sim 3.3 \mathrm{MtC} \mathrm{yr}^{-1}$ for the Mackenzie Shelf and delta, but a large fraction of this marine carbon seems to be rapidly recycled in both the water column and the sediment/water interface (Macdonald et al., 1998).

Based on $\delta^{13} \mathrm{C}$ bulk values, previous studies have estimated the total volume and the offshore extent of terrigenous organic matter preserved in the sediments of this region (Goñi et al., 2005; Magen et al., 2010). The issue of $\delta^{13} \mathrm{C}$ end-member determination is particularly problematic in this area (Amiel and Cochran, 2008; Belt et al., 2008; Forest et al., 2007), because there are other sources of POC than those derived from terrestrial vegetation and marine productivity. In particular, the fossil contribution from the river drainage basins may represent a significant source of POC with intermediate $\delta^{13} \mathrm{C}$ values, and the algal productivity in the rivers could be a source of $\delta^{13} \mathrm{C}$-depleted POC (Goñi et al., 2005). Mass balance studies using lipid $\delta^{13} \mathrm{C}$ and ${ }^{14} \mathrm{C}$ signatures indicated that $40-50 \%$ of the carbon buried in the Beaufort Sea was derived from the weathering of ancient sedimentary rock (Drenzek et al., 2007).

Compared to bulk geochemical analyses, where the issue of the $\delta^{13} \mathrm{C}$ marine end-member determination is particularly delicate, the examination of organic matter at molecular level can provide more specific information related to the carbon cycle, source identification and apportionment in the Beaufort Sea. Several studies from the 1980s have partially characterized the Mackenzie-Beaufort sea system using specific molecular compounds (Yunker et al., 1995, 2002, 2005, 2011; Belicka et al., 2004; Goñi et al., 2000, 2005; Drenzek et al., 2007). In particular, these studies all reported data on lipid biomarkers, which are well suited to evaluate the supply of terrigenous organic matter (Bouloubassi et al., 1997; Saliot et al., 2002), but only a few have combined these analyses with compound-specific isotope determinations (Drenzek et al., 2007; Goñi et al., 2005). The approach using the $\delta^{13} \mathrm{C}$ values of individual compounds is relevant since the isotope data provide additional evidence supporting the proposed origins of the biomarkers, and in certain cases this combination is essential for an accurate determination of the origin of the biomarkers. The present study combines a comprehensive list of biomarkers and compound-specific carbon isotope analysis on suspended particulate matter (SPM) and surface sediments collected in summer 2009. Here, the cou-

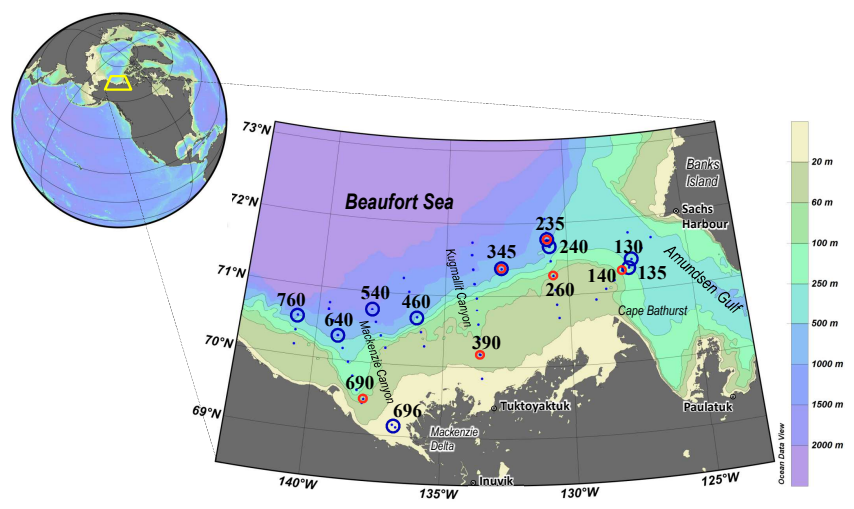

Fig. 1. Map of the Malina survey area. Sites of suspended particulate matter sampling: blue circles. Sites of sediment sampling: red circles.

pled data were used in the southeast Beaufort Sea to better understand the sources of carbon, the transport and the fate of organic matter from the Mackenzie River to the marine off-shore waters and surface sediments. Based on the contribution of different molecular markers (hydrocarbons, alcohols, sterols and fatty acids) characteristic of known organic matter sources and taking into account the different lability of the molecules, we evaluate and compare the relative importance of different organic pools, such as fresh algal, refractory algal, fossil, $\mathrm{C}_{3}$ terrestrial plants, bacterial and zooplankton material between the river SPM, marine offshore SPM and surface sediments. Additionally, a quantitative approach comparing the concentrations of allochthonous biomarkers normalized to the total organic carbon between marine sediments and river SPM allowed the reassessment of the fraction of allochthonous material preserved in the sediments of the Beaufort Sea.

\section{Material and methods}

\subsection{Study area and sampling}

This study was conducted in the southeast Beaufort Sea during summer 2009 (1 to 24 August 2009) on board the Canadian research icebreaker CCGS Amundsen from approximately latitudes 69 to $72^{\circ} \mathrm{N}$ and longitudes 125 to $145^{\circ} \mathrm{W}$ (Table 1, Fig. 1). The study was organized within the framework of the Malina project.

The Arctic Ocean, including the Beaufort Sea, is strongly structured in several vertical layers and is filled with water from the Atlantic and Pacific oceans as well as superficial waters influenced by river discharge and ice melting (Stein and Macdonald, 2004). The water masses comprise the polar mixed layer (PML, salinity $<31,0-50 \mathrm{~m}$ depth), which is influenced by fresh waters from the rivers and melting sea ice, the upper Pacific halocline (salinity: 31-33, 50-200 m depth) associated with a prominent nutrient maximum at salinities 
Table 1. List of stations where sampling of suspended particulate matter (SPM) and sediments were conducted. For sediment samples, total organic carbon (TOC) is also indicated.

\begin{tabular}{|c|c|c|c|c|c|}
\hline Station & Latitude (N) & Longitude (W) & Bottom depth (m) & Date & TOC $\left(\mathrm{mg} \mathrm{g}^{-1}\right)$ \\
\hline \multicolumn{6}{|l|}{ SPM } \\
\hline 760 & 70.55 & 140.8 & 560 & 12 Aug 2009 & \\
\hline 640 & 70.34 & 139.14 & 570 & 12 Aug 2009 & \\
\hline 540 & 70.75 & 137.89 & 1514 & 18 Aug 2009 & \\
\hline 460 & 70.68 & 136.05 & 470 & 19 Aug 2009 & \\
\hline 345 & 71.36 & 132.61 & 540 & 15 Aug 2009 & \\
\hline 240 & 71.67 & 130.74 & 459 & 6 Aug 2009 & \\
\hline 235 & 71.76 & 130.83 & 600 & 24 Aug 2009 & \\
\hline 135 & 71.31 & 127.46 & 227 & 21 Aug 2009 & \\
\hline 130 & 71.43 & 127.36 & 311 & 7 Aug 2009 & \\
\hline Mackenzie River (696/697) & 69.16 & 136.81 & $2.7 / 1.7$ & 13 Aug 2009 & \\
\hline \multicolumn{6}{|l|}{ Sediments } \\
\hline 690 & 69.49 & 137.94 & 55 & 1 Aug 2009 & 16.5 \\
\hline 390 & 70.18 & 133.57 & 47 & 1 Aug 2009 & 17.5 \\
\hline 345 & 71.40 & 132.64 & 577 & 16 Aug 2009 & 13.8 \\
\hline 235 & 71.76 & 130.77 & 576 & 22 Aug 2009 & 13.7 \\
\hline 260 & 71.27 & 130.61 & 60 & 5 Aug 2009 & 13.2 \\
\hline 140 & 71.28 & 127.78 & 154 & 7 Aug 2009 & 16.8 \\
\hline
\end{tabular}

of $\sim 33$, the lower Atlantic halocline (200-275 m depth) and the relatively warm $\left(>0{ }^{\circ} \mathrm{C}\right)$ and salty deep waters of Atlantic origin (salinity $>34.5,>275 \mathrm{~m}$ depth). The main hydrodynamic and trophic features for the different zones are described in more detail by Codispoti et al. (2005) and by Matsuoka et al. (2012). A particularly important feature of the Arctic Ocean is the strong perennial cold halocline, which isolates surface waters (and sea ice) from warm and salty Atlantic waters.

The sample set of the present study includes the following: (1) suspended particulate material (SPM) from the slope waters (200-1500 $\mathrm{m}$ bottom depth) and from the Mackenzie River and (2) superficial sediments from the Mackenzie Shelf $(<100 \mathrm{~m})$, the slope and from the mouth of the Amundsen Gulf. The Mackenzie Shelf is $\sim 150 \mathrm{~km}$ wide and is crossed by two submarine canyons: the broad, deep Mackenzie Canyon at about $138^{\circ} \mathrm{W}$, and the narrow, relatively shallow Kugmallit Canyon at about $134^{\circ}$ W. SPM samples were collected at the chlorophyll $a$ maximum depth, determined by a CTD probe equipped with a fluorometer, at the Pacific halocline layer ( $\sim 130 \mathrm{~m}$ depth) and at 200 and/or $300 \mathrm{~m}$ depth. One sample at site 130 was taken in the upper PML ( $3 \mathrm{~m}$ depth) in waters showing a salinity of 28.2.

For sampling suspended particles, Challenger Oceanics in situ pumps were used to filter large volumes $(400$ to $900 \mathrm{~L}$ ) of water, through a Nitex screen of $70 \mu \mathrm{m}$ and a precombusted $\left(550^{\circ} \mathrm{C}\right.$ ) microquartz filter (QMF, Sartorius) of $1 \mu \mathrm{m}$ pore size. Only the size fraction collected on the microquartz filter $(1-70 \mu \mathrm{m})$ was analysed. SPM from the Mackenzie River was obtained by collection of freshwater with a Zodiac and filtering on GF/F filters.
Sediments were sampled by means of a box corer. The top $5 \mathrm{~mm}$ of sediment were sampled with a metallic spatula and collected in a Teflon tube. Filters and sediments were stored frozen at $-80{ }^{\circ} \mathrm{C}$ until analysis in the laboratory.

Organic carbon was measured with a Vario EL elemental analyser (Elementar Analysensysteme $\mathrm{GmbH}^{\odot}$ ) after acidification of the filter aliquots and sediments with 1M H3PO4 (Miquel et al., 1994; Martin et al., 2009). Several runs of blanks (pre-combusted QMA filters) and standards (Acetanilide Merck pro analysis) were performed for calibration of carbon measurements.

The other auxiliary parameters corresponding to the suspended particulate matter samples (Table 2) were obtained from the Malina database, where data and methods are fully described. Briefly, temperature, pressure and salinity were measured using a Seabird Fastcast SBE-49. Suspended particulate matter (SPM) was obtained following the method described in Doxaran et al. (2012). Total Chl $a$ was obtained by using the method described in Ras et al. (2008). Nutrient concentrations (nitrate, phosphate and silicate) were determined onboard using the methods described in Raimbault et al. (2008). The dissolved $\mathrm{CO}_{2}$ concentration was derived from alkalinity, $\mathrm{pH}$, temperature, salinity and the concentrations of silicate and phosphate using the CO2SYS program developed for $\mathrm{CO}_{2}$ system (Lewis and Wallace, 1998). Total alkalinity $\left(A_{\mathrm{T}}\right)$ of water samples was measured by open-cell potentiometric titration (Mucci et al., 2010), and pH measurements on board were measured as described in Lansard et al. (2012). 
Table 2. Bulk biochemical parameters corresponding to the suspended particulate matter (SPM) samples in the Beaufort Sea and Mackenzie River. Stations are identified by their number (e.g. 130) followed by the sampling depth (e.g. 3).

\begin{tabular}{|c|c|c|c|c|c|c|c|c|c|c|}
\hline $\begin{array}{l}\text { Locations- } \\
\text { Depth (m) }\end{array}$ & $\begin{array}{r}\text { Depth } \\
(\mathrm{m})\end{array}$ & $\begin{array}{r}\text { Temperature } \\
\left({ }^{\circ} \mathrm{C}\right)\end{array}$ & Salinity & $\begin{array}{r}\mathrm{C}_{\mathrm{org}} \\
\left(\mu \mathrm{g} \mathrm{L}^{-1}\right)\end{array}$ & $\begin{array}{r}\mathrm{SPM} \\
\left(\mathrm{mg} \mathrm{L}^{-1}\right)\end{array}$ & $\begin{array}{r}\text { Total Chl } a \\
\left(\mathrm{mg} \mathrm{m}^{-3}\right)\end{array}$ & $\begin{array}{l}\mathrm{NO}_{3} \\
(\mu \mathrm{M})\end{array}$ & $\begin{array}{l}\mathrm{PO}_{4} \\
(\mu \mathrm{M})\end{array}$ & $\begin{array}{r}\mathrm{SiOH}_{4} \\
(\mu \mathrm{M})\end{array}$ & $\begin{array}{r}{\left[\mathrm{CO}_{2 \mathrm{aq}}\right]} \\
\left(\mu \mathrm{mol} \mathrm{kg}{ }^{-1}\right)\end{array}$ \\
\hline $130-3$ & 3 & 4.63 & 28.22 & 42.45 & 0.20 & 0.14 & 0.01 & 0.60 & 3.12 & 20.6 \\
\hline $130-130$ & 130 & -1.39 & 33.07 & 5.84 & & 0.03 & 5.45 & 1.27 & 1.92 & 40.3 \\
\hline $130-200$ & 200 & -0.99 & 33.99 & 6.25 & & 0.01 & 12.89 & 1.44 & 25.21 & 34.1 \\
\hline $135-40$ & 40 & -0.82 & 31.26 & 11.17 & 0.07 & 0.10 & 0.01 & 0.79 & 3.71 & 17.3 \\
\hline $135-70$ & 70 & -1.26 & 31.75 & 11.10 & 0.07 & 0.21 & 6.67 & 1.29 & 15.49 & 20.8 \\
\hline $135-85$ & 85 & -1.27 & 32.21 & 7.58 & & 0.15 & 8.12 & 1.50 & 18.67 & 33.3 \\
\hline $135-145$ & 145 & -1.41 & 33.07 & 5.92 & & $<0.042$ & 15.17 & 1.82 & 33.08 & 38.6 \\
\hline $235-70$ & 70 & -1.12 & 31.89 & 12.33 & 0.07 & 0.27 & & & & 25 \\
\hline $235-85$ & 85 & -1.30 & 32.37 & 7.69 & & 0.13 & & & & 34 \\
\hline $235-145$ & 145 & -1.37 & 32.75 & 3.81 & & $<0.0087$ & & & & 39.4 \\
\hline $235-200$ & 200 & -0.07 & 34.55 & 3.18 & & & & & & 24.5 \\
\hline $240-70$ & 70 & -1.20 & 32.11 & 8.37 & 0.60 & 0.12 & 9.62 & 1.40 & 23.61 & 28.6 \\
\hline $240-130$ & 130 & -1.41 & 33.03 & 6.44 & & 0.03 & 13.20 & 1.59 & 33.41 & 40.3 \\
\hline $240-200$ & 200 & -0.71 & 34.24 & 9.34 & & 0.02 & & & & 28.1 \\
\hline $345-60$ & 60 & -1.15 & 31.33 & 18.81 & 0.15 & 0.52 & 2.80 & 1.01 & 9.15 & 18.7 \\
\hline $345-85$ & 85 & -1.24 & 32.19 & 5.39 & & 0.16 & 9.91 & 1.54 & 23.40 & 30.4 \\
\hline $345-145$ & 145 & -1.40 & 33.16 & 1.10 & & & & & & 38.3 \\
\hline $345-200$ & 200 & -0.62 & 34.28 & 2.77 & & & & & & 27.1 \\
\hline $460-70$ & 70 & -1.14 & 31.85 & 12.93 & 0.07 & 0.53 & & & & 26.3 \\
\hline $460-130$ & 130 & -1.43 & 33.09 & 4.36 & & 0.01 & & & & 40.9 \\
\hline $460-200$ & 200 & -0.75 & 34.19 & 8.14 & & & & & & 27.7 \\
\hline $460-300$ & 300 & & & 6.07 & & & & & & \\
\hline $540-70$ & 70 & -1.15 & 31.82 & 6.61 & 0.06 & 0.44 & 6.06 & 2.20 & 14.04 & 24.8 \\
\hline $540-90$ & 90 & -1.36 & 32.26 & 7.48 & & 0.06 & 10.22 & 2.75 & 22.43 & 33.5 \\
\hline $540-200$ & 200 & -0.43 & 34.38 & 2.76 & & $<0.0054$ & 12.26 & 1.74 & 12.71 & 24.9 \\
\hline $640-70$ & 70 & -1.09 & 30.48 & 11.71 & 0.05 & 0.15 & & & & $\sim 21.7$ \\
\hline $640-130$ & 130 & & & 6.19 & & $<0.048$ & & & & \\
\hline $640-200$ & 200 & & & 3.97 & & $<0.0136$ & & & & \\
\hline $760-130$ & 130 & -1.43 & 33.01 & 3.82 & & 0.01 & 15.65 & 1.93 & 37.19 & 39.2 \\
\hline $760-200$ & 200 & & & 4.28 & & & & & & \\
\hline $760-300$ & 300 & 0.29 & 34.67 & 3.24 & & & & & & 24.4 \\
\hline Mackenzie River & 0.5 & 10.08 & 0.23 & 1210 & 111.50 & 3.16 & 3.31 & 0.02 & 64.17 & \\
\hline
\end{tabular}

\subsection{Lipid extraction}

Filters containing the suspended particles and freeze-dried sediments were spiked with internal standards $\left(n-\mathrm{C}_{24} \mathrm{D}_{50}\right.$, friedeline, $5 \alpha$-androstan- $3 \beta$-ol and cholanic acid), and lipids extracted with $40 \mathrm{~mL}$ of a mixture of $\mathrm{CH}_{2} \mathrm{Cl}_{2} / \mathrm{MeOH}(3: 1)$ in a microwave oven at $70^{\circ} \mathrm{C}$ for $15 \mathrm{~min}$. Isolation of the neutral and acid lipid fractions was performed following the method of Tolosa and de Mora (2004a). Extractable lipids were saponified using $1 \mathrm{~mL}$ of $\mathrm{KOH} 6 \%$ in methanol/water $(80: 20)$ plus $1 \mathrm{~mL}$ of Milli-Q water $\left(80^{\circ} \mathrm{C}, 1 \mathrm{~h}\right)$. The neutral fraction was then recovered with $n$-hexane and subjected to fractionation by HPLC on a normal phase column $(\mathrm{Nu}-$ cleosil column, $20 \mathrm{~cm} \times 0.4 \mathrm{~cm}$ i.d. $5 \mu \mathrm{m}$ ) to isolate aliphatic hydrocarbons (F1), polycyclic aromatic hydrocarbons (F2), ketone compounds (F3) and sterol and alcohol fraction (F4). $\mathrm{F} 1$ and $\mathrm{F} 2$ were combined for the total hydrocarbon analysis. Saponified solutions were acidified with $1 \mathrm{~mL}$ of $\mathrm{HCl} 6 \mathrm{~N}$ to $\mathrm{pH} 2$, and the fatty acids were extracted with hexane: ethyl acetate $(9: 1)$.

\subsection{Gas chromatography}

The sterol fraction was treated with bis-(trimethylsilyl)trifluoroacetamide (BSTFA) $\left(200 \mu \mathrm{L}, 70^{\circ} \mathrm{C}, 1 \mathrm{~h}\right)$ to convert the alcohols and sterols to their corresponding trimethylsilyl ethers. The acid fraction was derivatized by transesterifying 
the lipid extract with $500 \mu \mathrm{L}$ of $20 \% \mathrm{BF}_{3}$ in methanol at $80^{\circ} \mathrm{C}$ for $1 \mathrm{~h}$.

Quantification of neutral compounds was performed on a Hewlett-Packard HP 7890 A with a flame ionisation detector (FID) and a splitless injector. The column was a HP-5 $(30 \mathrm{~m} \times 0.25 \mathrm{~mm}$ i.d. $\times 0.25 \mu \mathrm{m}$ film thickness), and injector and detector temperatures were $270^{\circ} \mathrm{C}$ and $320^{\circ} \mathrm{C}$, respectively. The oven temperature was programmed at $4{ }^{\circ} \mathrm{C} \mathrm{min}-1$ from $60^{\circ} \mathrm{C}$ to $310^{\circ} \mathrm{C}$, then held $20 \mathrm{~min}$.

Quantification of the acidic compounds was carried out with a Hewlett-Packard HP 5890 series II equipped with a FID and on-column injector. The DB$23(30 \mathrm{~m} \times 0.32 \mathrm{~mm} \times 0.25 \mu \mathrm{m})$ column was pre-connected with a press-fit connector to a $0.32 \mathrm{~mm}$ i.d., deactivated, fused silica capillary. Helium was the carrier gas $\left(1.2 \mathrm{~mL} \mathrm{~min}^{-1}\right)$. The $\mathrm{GC}$ oven for the DB-23 column was programmed from $60^{\circ} \mathrm{C}(0.5 \mathrm{~min}$ hold $)$ to $250^{\circ} \mathrm{C}$ at $6^{\circ} \mathrm{C} \min ^{-1}$.

Aliphatic hydrocarbons, sterols and fatty acids were quantified by internal standards $\left(\mathrm{C}_{24} \mathrm{D}_{50}, 5 \alpha\right.$-androstan- $3 \beta$-ol, and cholanic acid, respectively). Confirmation of peak identity was obtained using GC with mass spectrometric detection (GC-MS) (Hewlett-Packard 5889B MS "Engine") operated in the electron impact at $70 \mathrm{eV}$. It was equipped with a HP5-MS column $(30 \mathrm{~m} \times 0.25 \mathrm{~mm}$ i.d., $0.25 \mu \mathrm{m}$ thick). Helium was the carrier gas. The oven temperature was programmed from $60{ }^{\circ} \mathrm{C}$ to $290{ }^{\circ} \mathrm{C}$ at $4{ }^{\circ} \mathrm{C} \mathrm{min}^{-1}$. Compound identification was made according to their mass spectra and the retention times of standards.

\subsection{Compound-specific isotope analysis}

The lipid biomarkers were analysed for their stable carbon isotope composition using an HP 5890 GC equipped with a HP 7673 autoinjector and interfaced through a combustion furnace with a Finnigan MAT Delta $\mathrm{C}$ isotope ratio mass spectrometer (GC/C/IRMS).

The GC/C/IRMS was equipped with a $100 \%$ methylpolysiloxane fused silica column (Ultra-1, $50 \mathrm{~m} \times 0.32 \mathrm{~mm}$ i.d.; $0.5 \mu \mathrm{m}$ film thickness) pre-connected with a press-fit connector (Supelco, France) to a $0.32 \mathrm{~mm}$ i.d., deactivated, fused silica capillary retention gap of $5 \mathrm{~m}$. Injections of $2 \mu \mathrm{L}$ in isooctane were made via an on-column injector. The $\mathrm{GC}$ oven was programmed from 60 to $100^{\circ} \mathrm{C}$ at $10^{\circ} \mathrm{C} \mathrm{min}-1$, then to $310^{\circ} \mathrm{C}$ at $4{ }^{\circ} \mathrm{C} \mathrm{min}^{-1}$ and maintained at $310^{\circ} \mathrm{C}$ for $40 \mathrm{~min}$. Values reported were determined at least from triplicates to calculate the average and standard deviation. All $\delta^{13} \mathrm{C}$ values are reported in the delta notation relative to the Pee Dee Belemnite (PDB) standard as follows:

$\delta^{13} \mathrm{C} \% o=\left[\left({ }^{13} \mathrm{C} /{ }^{12} \mathrm{C}\right)_{\text {sample }} /\left({ }^{13} \mathrm{C} /{ }^{12} \mathrm{C}\right)_{\mathrm{PDB}}-1\right] \times 10^{3}$.

Corrections for the isotopic change introduced in the derivatization of sterols, fatty alcohols, and fatty acids were determined through derivatization of standards of known isotopic composition applying the equation of Jones et al. (1991). Cholesterol, $\mathrm{C}_{18: 0}$ fatty acid and $\mathrm{C}_{18: 0}$ FAME, methanol (with $\mathrm{BF}_{3}$ ) and BSTFA of known isotopic carbon composition (measured by elemental analyser coupled to isotope ratio mass spectrometer) were used to calibrate the GC/C/IRMS and correct the isotopic change introduced by the derivatization. The surrogate standards, $5 \alpha$-androstan- $3 \beta$-ol, cholanic acid and the GC internal standard friedeline of known isotopic composition served as internal isotopic standards.

Most of the values reported here correspond to the $\delta^{13} \mathrm{C}$ values of peak abundances higher than $0.5 \mathrm{~V}(\mathrm{~m} / z$ 44) where the standard deviation (S.D) was comparable to the instrument specifications $\left(0.5 \%\right.$ ) . We also present some $\delta^{13} \mathrm{C}$ values of peak abundances $\geq 0.3 \mathrm{~V}$ and $\leq 0.5 \mathrm{~V}$ with slightly higher variability (S.D. $<2 \%$ ), which was validated by standards within this range.

\subsection{Background on lipid biomarker origins}

Hydrocarbons from both natural and anthropogenic sources are very common in the environment. Among biogenic hydrocarbons, the $n$-alkanes exhibit a strong odd carbonnumber predominance in living organisms where the carbonnumber distributions vary depending on the source organism. Higher terrestrial plants are dominated by the longchain $\left(\mathrm{C}_{25}-\mathrm{C}_{33}\right) n$-alkanes, lower terrestrial plants by the medium-chain $\left(\mathrm{C}_{23}-\mathrm{C}_{25}\right) n$-alkanes and aquatic algae by the short-chain $n$-alkanes $\left(\mathrm{C}_{17}-\mathrm{C}_{21}\right)$ (Volkman et al., 1992; Baas et al., 2000). Other biogenic hydrocarbons include the polyunsaturated straight-chain alkenes, $n$ - $\mathrm{C}_{21: 6}$ and related isomers $\left(n-\mathrm{C}_{21: 5}, n-\mathrm{C}_{21: 4}\right)$ derived from autotrophic marine and freshwater plankton (Volkman et al., 1992), the $\mathrm{C}_{25}$ monounsaturated hydrocarbon $\left(\mathrm{IP}_{25}\right)$ used as a sea ice algae proxy (Belt et al., 2007) and retene, a diagenetic polycyclic aromatic hydrocarbon (PAH) from higher plants (Simoneit, 1977). In contrast to these biogenic sources, petroleum sources usually show a wide distribution range of $n$-alkanes with no predominance of odd or even carbon numbers. We use the carbon preference index (CPI) defined as the sum of the odd carbon-numbered alkanes added to the sum of the even carbon-numbered alkanes to characterize the sources of $n$-alkanes (Bray and Evans, 1961). In fact, extant plants typically show CPIs $>5$ (Rielley et al., 1991) while petroleumderived $n$-alkanes have CPI values around 1 (Wang et al., 2003). Whenever both sources are mixed, terrestrial wax $n$ alkanes $\left(\mathrm{C}_{25}-\mathrm{C}_{31}\right)$ are calculated by subtracting the fossil contribution resulting from the average of the next higher and lower even carbon-numbered homologues as follows: wax $n$ $\mathrm{C}=\left[\mathrm{C}_{n}\right]-0.5\left[\mathrm{C}_{(n+1)}+\mathrm{C}_{(n-1)}\right]$ (Simoneit et al., 1990). The presence of an unresolved complex mixture (UCM) defined as a hump in the hydrocarbon chromatograms is also a diagnostic tool of petroleum/fossil sources (Volkman et al., 1992). 
Among alcohols, phytol is a marker for phototrophic organisms (Baker and Louda, 1983). Short-chain $\left(n-\mathrm{C}_{14}\right.$ $\mathrm{C}_{20}$ ) alcohols $(\mathrm{SCOH})$ and short-chain monounsaturated alcohols (SCMUOH) might have multiple microbial sources (Robinson et al., 1984b), and long-chain $\left(n-\mathrm{C}_{22}-\mathrm{C}_{30}\right)$ even carbon-numbered $n$-alcohols (LCOH) are markers of terrestrial higher plant waxes (Rieley et al., 1991), and long-chain $\left(\mathrm{C}_{20}-\mathrm{C}_{24}\right)$ monounsaturated fatty alcohols (LCMUOH), biomarkers typical of zooplankton (Lee et al., 2006). Finally, $\alpha$-amyrin (Olean-12-en-3 $\alpha$-ol), is a specific triterpenoid for angiosperms and also present in peat (Hernes and Hedges, 2004).

Sterols occur in all eukaryotic organisms, and the specificity of these compounds for different phytoplankton groups and vascular plants is well known (Volkman, 1986, 2003). The 24-methylcholesta-5,22(E)dien-3 $\beta$-ol $\left(\mathrm{C}_{28} \Delta^{5,22}\right.$, brassicasterol), 24-methylcholesta5,24(28)-dien-3 $\beta$-ol $\left(\mathrm{C}_{28} \Delta^{5,24(28)}\right)$ and 24-ethylcholest-5en-3 $\beta$-ol (sitosterol, $\mathrm{C}_{29} \Delta^{5}$ ) are common lipids in diatoms (Barrett et al., 1995, Volkman et al., 1998); $\mathrm{C}_{28} \Delta^{5,24(28)}$ and the $Z$ isomer of fucosterol (isofucosterol, 24-ethylcholesta-5, 24(28)(Z)-dien-3 $\beta$-ol; $\mathrm{C}_{29} \Delta^{5,24(28)}$ ) are typical of prasinophytes (Volkman et al., 1994). Dinosterol $\left(4 \alpha-23,24\right.$-trimethylcholest-22(E)-en-3 $\beta$-ol $\left(\mathrm{C}_{30} \Delta^{22}\right)$ is commonly used as a biomarker for dinoflagellates (Robinson et al., 1994a) and 27-nor-24-methylcholesta-5, 22(E)dien-3 $\beta$-ol (norC $27 \Delta^{5,22}$ ) together with $\mathrm{C}_{28} \Delta^{5,22}$ predominate in the marine dinoflagellate Gymnodinium simplex (Goad and Withers, 1982) and in marine invertebrates (Volkman et al., 1981). Cholest-5-en-3 $\beta$-ol (cholesterol, $\mathrm{C}_{27} \Delta^{5}$ ) is considered a typical marker for zooplankton-derived organic matter supply, but it is also present in many classes of algae (Harvey et al., 1987). The 24-methylcholesta-5-en-3 $\beta$-ol $\left(\mathrm{C}_{28} \Delta^{5}\right)$, 24-ethylcholesta-5, 22(E)-dien-3 $\beta$-ol $\left(\mathrm{C}_{29} \Delta^{5,22}\right)$ and sitosterol have often been considered as terrestrial markers, but they are also produced by both phytoplankton and aquatic plants (e.g. Volkman, 1986, 2003).

Fatty acids (FAs) are among the most abundant lipid biomarkers. Different group classes are distinguishable: the linearly saturated are used to separate marine from terrigenous sources through short-chain $\mathrm{C}_{14}-\mathrm{C}_{18}$ (SCFA) and longchain $\mathrm{C}_{22}-\mathrm{C}_{30}$ (LCFA), respectively (Grimalt and Albaigés, 1990). Branched fatty acids (BrFA), composed of iso- and anteiso-branched compounds with odd chain lengths (e.g. $\mathrm{C}_{15}, \mathrm{C}_{17}$ ), are used as bacterial markers (Volkman et al., 1980). Vaccenic acid $\left(C_{18: 1 \omega 7}\right)$ is considered to be an indicator of bacterial input when $18: 1 \omega 9 / 18: 1 \omega 7<1$. However when this isomer ratio $>1$, then it suggests a dominant phytoplanktonic source (Thoumelin et al., 1997). Monounsaturated long-chain $\mathrm{C}_{22}-\mathrm{C}_{30}$ (LCMUFA) are typical of zooplankton (Lee et al., 2006). Polyunsaturated fatty acids (PUFA) are used as plankton biomarkers. Their presence indicates a fresh algal input, because they are not resistant to degradation processes. Among the PUFA, the $\mathrm{C}_{18: 5 w 3}$ and $\mathrm{C}_{18: 4 w 3}$ are abundant in flagellates (green algae and cryp- tomonads), $\mathrm{C}_{20: 5 w 3}$ predominates in many diatom species and $\mathrm{C}_{22: 6 w 3}$ dominates in dinoflagellates and flagellates (crytomonads) (Dalsgaard et al., 2003).

We estimated the relative contributions of organic matter $(\mathrm{OM})$ constituents by grouping the different molecular lipid biomarkers into their different sources (fossil, algal, zooplankton, bacterial and terrestrial). We also took into account the different lability of the molecules to discern between fresh/labile and refractory/detrital algae. We assigned PUFA, phytol, $\mathrm{IP}_{25}, n-\mathrm{C}_{21: 6}, \mathrm{C}_{28} \Delta^{5,24(28),} \mathrm{C}_{29} \Delta^{5,24(28)}$ within the fresh/algal component, because these molecules are more labile bearing double bonds or oxygenated functionalities compared to the higher stability of the rest of biomarkers studied. These criteria yielded the following components: fossil (UCM, and petroleum hydrocarbons), fresh/labile algal (PUFA, phytol, $\mathrm{IP}_{25}, n-\mathrm{C}_{21: 6}, \mathrm{C}_{28} \Delta^{5,24(28)}$, $\mathrm{C}_{29} \Delta^{5,24(28)}$ ), refractory/detrital algal (SCFA, SCMUFA, rest of sterols, biogenic alkanes, SCOH, SCMUOH), zooplankton (LCMUFA, LCMUOH), bacterial (branched FAs) and $\mathrm{C}_{3}$ terrestrial plants (LCFA, LCOH, wax $n$-alkanes). We stress that this is an empirical approach because some of the compounds, such as sitosterol, might derive from more than one source (algal and/or terrestrial). Hence, only relative changes among the stations and depths were evaluated.

\section{Results}

Table 2 describes the biochemical parameters accompanying the SPM samples from the slope waters of the Beaufort Sea and from the Mackenzie River. For clarification, the first number in the codes, e.g. 640-70, refers to the station (640), whereas the second number after the dash indicates the water depth sampled $(70 \mathrm{~m})$. The sample $130-3$ was the only one obtained in waters, which exhibited clear influence from the rivers and/or melting sea ice (salinity $<29$ ). For samples from the depth of chlorophyll and POC maximum (60-70 m), the seawater showed salinity values $\sim 31-32$ and nitrate concentrations from 2.8 to $9.6 \mu \mathrm{M}$, consistent with the nutrientreplete Pacific halocline water mass. At $130 \mathrm{~m}$ depth, salinity values of $\sim 33$, high concentrations of nitrates $(\sim 13-15 \mu \mathrm{M}$, except site 130) and $\mathrm{CO}_{2}$, and very low values of POC and total chlorophyll $a$ were measured. Finally at $200 \mathrm{~m}$ and below, salinities were highest $(\geq 34)$ showing a typical Atlantic water origin (Matsuoka et al., 2012).

\subsection{Hydrocarbons}

Tables 3 and 4 summarize the hydrocarbons found in the SPM and sediments. All marine suspended particulate samples except for the most superficial one at $3 \mathrm{~m}$ depth showed traces of $n$-alkanes from $\mathrm{C}_{23}$ to $\mathrm{C}_{33}$ with no odd/even predominance (CPI 1). The hydrocarbon fraction of the SPM of the Mackenzie River and that from the surficial sediments (Table 4) showed a wide distribution range of quantifiable 
Table 3. Selected hydrocarbon concentrations and diagnostic ratios in suspended particle samples from the Beaufort Sea.

\begin{tabular}{|c|c|c|c|c|c|c|c|}
\hline $\begin{array}{l}\text { Locations- } \\
\text { Depth (m) }\end{array}$ & $\begin{array}{r}n-\mathrm{C}_{17} \\
\left(n g \mathrm{~L}^{-1}\right)\end{array}$ & $\begin{array}{r}\sum n-\mathrm{C}_{21: x} \\
\left(n g \mathrm{~L}^{-1}\right)\end{array}$ & $\begin{array}{r}\text { Retene } \\
\left(\mathrm{ng} \mathrm{L}^{-1}\right)\end{array}$ & $\begin{array}{r}\text { Wax }\left(\mathrm{C}_{25}-\mathrm{C}_{31}\right) \\
\left(\mathrm{ng} \mathrm{L}^{-1}\right)\end{array}$ & $\begin{array}{c}n \text {-alkanes } \\
\left(\text { ng L }^{-1}\right)\end{array}$ & $\begin{array}{r}\mathrm{UCM} \\
\left(\mathrm{ng} \mathrm{L}{ }^{-1}\right)\end{array}$ & $\begin{array}{r}\text { CPI } \\
\left(\mathrm{C}_{23}-\mathrm{C}_{35} / \mathrm{C}_{22}-\mathrm{C}_{34}\right) \\
\end{array}$ \\
\hline $130-3$ & $<0.32$ & 8.46 & $<0.02$ & 9.20 & 28.32 & n.d. & 2.73 \\
\hline $130-130$ & $<0.14$ & 0.78 & $<0.02$ & $<0.3$ & 1.64 & n.d. & 1.63 \\
\hline $130-200$ & $<0.14$ & 0.29 & $<0.01$ & $<0.3$ & 2.50 & n.d. & 1.32 \\
\hline $135-40$ & $<1.14$ & 1.60 & $<0.02$ & $<0.3$ & 2.06 & n.d. & 0.80 \\
\hline $135-70$ & $<0.81$ & 2.90 & $<0.02$ & $<0.3$ & 1.04 & n.d. & 1.15 \\
\hline $135-85$ & $<0.73$ & 1.94 & $<0.02$ & $<0.3$ & 1.23 & n.d. & 1.10 \\
\hline $135-145$ & $<0.85$ & 0.23 & $<0.02$ & $<0.3$ & 1.62 & n.d. & 1.07 \\
\hline $235-70$ & $<0.93$ & 3.80 & $<0.02$ & $<0.3$ & 0.85 & n.d. & 1.65 \\
\hline $235-85$ & $<0.79$ & 2.31 & $<0.02$ & $<0.3$ & 1.65 & n.d. & 1.15 \\
\hline $235-145$ & $<1.67$ & 0.12 & $<0.01$ & $<0.3$ & 2.60 & n.d. & 1.48 \\
\hline $235-200$ & $<0.73$ & 0.12 & $<0.01$ & $<0.3$ & 1.64 & n.d. & 1.20 \\
\hline $240-70$ & $<0.58$ & 2.97 & $<0.01$ & $<0.3$ & 2.75 & n.d. & 1.03 \\
\hline $240-130$ & $<0.70$ & 0.15 & $<0.04$ & $<0.3$ & 12.14 & n.d. & 1.25 \\
\hline $240-200$ & $<0.65$ & 1.23 & $<0.04$ & 0.38 & 19.16 & n.d. & 1.11 \\
\hline $345-60$ & $<0.18$ & 10.41 & $<0.01$ & $<0.3$ & 1.14 & n.d. & 1.39 \\
\hline $345-85$ & $<1.03$ & 1.30 & $<0.01$ & $<0.3$ & 0.45 & n.d. & 1.09 \\
\hline $345-145$ & $<0.13$ & 0.04 & $<0.01$ & $<0.3$ & 0.28 & n.d. & 2.69 \\
\hline $345-200$ & $<0.17$ & 0.08 & $<0.01$ & $<0.3$ & 1.10 & n.d. & 1.21 \\
\hline $460-70$ & $<0.13$ & 4.78 & $<0.01$ & $<0.3$ & 0.29 & n.d. & n.d. \\
\hline $460-130$ & $<0.13$ & 0.14 & $<0.01$ & $<0.3$ & 1.57 & n.d. & 1.56 \\
\hline $460-200$ & $<0.12$ & 0.19 & $<0.01$ & $<0.3$ & 2.27 & n.d. & 0.80 \\
\hline $460-300$ & $<0.45$ & 0.00 & $<0.01$ & $<0.3$ & 1.27 & n.d. & 2.30 \\
\hline $540-70$ & $<0.17$ & 1.91 & $<0.01$ & $<0.3$ & 1.24 & n.d. & 1.00 \\
\hline $540-90$ & $<0.17$ & 1.39 & $<0.01$ & $<0.3$ & 0.98 & n.d. & 1.36 \\
\hline $540-200$ & $<0.10$ & 0.11 & $<0.01$ & $<0.3$ & 1.86 & n.d. & 1.14 \\
\hline $640-70$ & $<0.95$ & 0.81 & $<0.04$ & $<0.3$ & 16.82 & n.d. & 1.16 \\
\hline $640-130$ & $<0.12$ & 0.48 & $<0.01$ & $<0.3$ & 2.34 & n.d. & 1.42 \\
\hline $640-200$ & $<0.10$ & 0.23 & $<0.01$ & $<0.3$ & 0.78 & n.d. & 1.55 \\
\hline $760-130$ & $<0.18$ & 0.12 & $<0.01$ & $<0.3$ & 1.11 & n.d. & 1.02 \\
\hline $760-200$ & $<0.20$ & 0.23 & $<0.01$ & $<0.3$ & $<0.5$ & n.d. & n.d. \\
\hline $760-300$ & $<0.17$ & 0.04 & $<0.01$ & $<0.3$ & 1.19 & n.d. & 1.10 \\
\hline Mackenzie & 129 & 50 & 14 & 231 & 1547 & 8376 & 2.39 \\
\hline
\end{tabular}

$n-\mathrm{C}_{12}$ to $n-\mathrm{C}_{35}$ alkanes, which is overlying a moderate unresolved complex mixture (UCM), typical of fossil/petrogenic sources. Algal and photosynthetic bacterial hydrocarbons, characterized by low, odd-numbered carbon $n$-alkanes $(n$ $\mathrm{C}_{17}$ ), were only predominant in the Mackenzie River water and sediments. Similarly, the long-chain homologues $\left(n-\mathrm{C}_{27}\right.$, $\left.n-\mathrm{C}_{29}, n-\mathrm{C}_{31}\right)$ derived from terrestrial higher plant waxes and the mid-chain $n$-alkanes of odd carbon-numbered $\left(n-\mathrm{C}_{23}, n\right.$ $\mathrm{C}_{25}$ ) derived from lower plants were only detected in the sediments, in the superficial suspended particles of site 130-3 and in the freshwater sample of the Mackenzie River.

Other compounds derived from autotrophic marine and freshwater plankton, e.g. the polyunsaturated straight-chain alkenes, $n-\mathrm{C}_{21: 6}$ and related isomers $\left(n-\mathrm{C}_{21: 5}, n-\mathrm{C}_{21: 4}\right)$ (Volk- man et al., 1992), were found in all SPM and sediments. Higher concentrations were measured in the deep chlorophyll maxima (DCM) and in the inner shelf sediments.

The $\mathrm{C}_{25}$ monounsaturated hydrocarbon $\left(\mathrm{IP}_{25}\right)$ from sea ice diatoms (Belt et al., 2007) was found in all sediment samples with concentrations ranging from 44 to $235 \mathrm{ng} \mathrm{g}^{-1}$, the highest concentration being measured at site 390 of the east and the lowest at site 690 off the west river mouth.

Retene, a diagenetic polycyclic aromatic hydrocarbon (PAH) from higher plants, was only detected in sediments and in the riverine particulate matter $\left(14 \mathrm{ng} \mathrm{L}^{-1}\right)$. Higher concentrations were found in the riverine particulate matter and shelf sediments. 
Table 4. Selected hydrocarbon concentrations and diagnostic ratios in sediment samples from the Beaufort Sea

\begin{tabular}{|c|c|c|c|c|c|c|c|c|c|}
\hline $\begin{array}{l}\text { Locations- } \\
\text { Depth (m) }\end{array}$ & $\begin{array}{r}n-\mathrm{C}_{17} \\
\left(\mathrm{ng} \mathrm{g}^{-1}\right)\end{array}$ & $\begin{array}{r}\sum n-\mathrm{C}_{21: x} \\
\left(\mathrm{ng} \mathrm{g}^{-1}\right)\end{array}$ & $\begin{array}{r}\mathrm{IP}_{25} \\
\left(\text { ng g }^{-1}\right)\end{array}$ & $\begin{array}{r}\text { Retene } \\
\left(\mathrm{ng} \mathrm{g}^{-1}\right)\end{array}$ & $\begin{array}{r}\text { Wax }\left(\mathrm{C}_{25}-\mathrm{C}_{31}\right) \\
\left(\mathrm{ng} \mathrm{g}^{-1}\right)\end{array}$ & $\begin{array}{r}n \text {-alkanes } \\
\left(\text { ng g }^{-1}\right)\end{array}$ & $\begin{array}{r}\mathrm{UCM} \\
\left(\mathrm{ng} \mathrm{g}^{-1}\right)\end{array}$ & $\mathrm{CPI}\left(\mathrm{C}_{23}-\mathrm{C}_{35} / \mathrm{C}_{22}-\mathrm{C}_{34}\right)$ & $\mathrm{UCM} / n$-alkanes \\
\hline $390-47$ & 466 & 950 & 235 & 57 & 1031 & 7131 & 47856 & 2.4 & 6.7 \\
\hline $690-55$ & 392 & 748 & 44 & 52 & 1166 & 6259 & 34781 & 3.0 & 5.6 \\
\hline $260-60$ & 141 & 218 & 164 & 41 & 574 & 2658 & 15486 & 3.1 & 5.8 \\
\hline $140-154$ & 163 & 418 & 185 & 21 & 645 & 3013 & 19684 & 3.0 & 6.5 \\
\hline $235-576$ & 225 & 66 & 127 & 32 & 577 & 3667 & 24503 & 2.7 & 6.7 \\
\hline $345-577$ & 211 & 50 & 99 & 29 & 701 & 3762 & 20983 & 2.7 & 5.6 \\
\hline
\end{tabular}

\subsection{Alcohols}

Tables 5 and 6 summarize the alcohol concentrations found in the SPM and sediments. Phytol and short-chain $n-\mathrm{C}_{14}-\mathrm{C}_{20}$ alcohols $(\mathrm{SCOH})$ were the major compounds identified in all marine SPM samples. The concentrations of phytol, a nonspecific marker for phototrophic organisms, if compared at the depth of chlorophyll and POC maxima, were highest at sites 345,460 , and 235 , and are consistent with the chlorophyll data (Table 2). Phytol concentration also decreased from surface to depth as expected due to degradation. Within the SPM samples, the long-chain $n$-alcohols $\left(n-\mathrm{C}_{22}\right.$ to $\left.n-\mathrm{C}_{30}\right)$ derived from terrestrial higher plant waxes $(\mathrm{LCOH})$ were only predominant in the Mackenzie River and in the surface water of site 130. In all other samples, they were below detection limit $\left(<1 \mathrm{ng} \mathrm{L}^{-1}\right)$. Long-chain monounsaturated fatty alcohols (LCMUOH $\left(\mathrm{C}_{20}-\mathrm{C}_{24}\right)$, biomarkers typical of zooplankton, were found at high concentration levels in the deeper water of site 240 and in the river freshwater; their percentage composition relative to total alcohols increased with water column depth.

In sediment samples, the highest concentrations of phytol were measured in the shallow sediments close to the river mouth (sites 390 and 690), and the lowest values observed in the deeper sediments of the slope (235 and 345). LCOH exhibited higher concentrations at the nearshore stations ( $3 \mu \mathrm{g} \mathrm{g}^{-1}$, constituting 53 to $70 \%$ of $n$-alcohols) than at sediments offshore (1.4-2.2 $\mu \mathrm{g} \mathrm{g}^{-1}$ ), but the contribution of the latter to the $n$-alcohols was higher (>70\%). The sediment at site 140, located in the Cape Bathurst polynya region at the mouth of the Amundsen Gulf, showed intermediate concentration values of terrestrial $n$-alcohols $\left(1.8 \mu \mathrm{g} \mathrm{g}^{-1}\right)$, with contributions to $n$-alcohols similar to those of the inshore stations $(59 \%)$.

\subsection{Sterols}

Tables 7 and 8 summarize, respectively, the percentage composition of the major sterols and total sterol concentrations found in the SPM and in the sediments. Sterol distribution in suspended particles from the deep chlorophyll maximum was dominated by 27 -nor-24-methylcholesta-5, 22(E)-dien-3 $\beta$ ol $\left(\right.$ norC $\left._{27} \Delta^{5,22}\right), 24$-methylcholesta-5, 22(E)-dien-3 $\beta$-ol $\left(\mathrm{C}_{28} \Delta^{5,22}\right.$, brassicasterol), 24-methylcholesta-5, 24(28)- dien-3 $\beta$-ol $\left(\mathrm{C}_{28} \Delta^{5,24(28)}\right)$ and the $Z$ isomer of fucosterol (isofucosterol, $\quad 24$-ethylcholesta-5, 24(28)(Z)-dien-3 $\beta$-ol; $\left.\mathrm{C}_{29} \Delta^{5,24(28)}\right)$. Other minor sterols included cholesta$5,22(E)$-dien- $3 \beta$-ol $\left(\mathrm{C}_{27} \Delta^{5,22}\right)$, cholest-5-en- $3 \beta$-ol (cholesterol, $\left.\mathrm{C}_{27} \Delta^{5}\right)$, 24-methylcholesta-5-en-3 $\beta$-ol $\left(\mathrm{C}_{28} \Delta^{5}\right)$; 24-ethylcholesta-5, $22(E)$-dien-3 $\beta$-ol $\quad\left(\mathrm{C}_{29} \Delta^{5,22}\right), \quad 24$ ethylcholest-5-en- $3 \beta$-ol (sitosterol, $\mathrm{C}_{29} \Delta^{5}$ ), the $4 \alpha$ 23,24 -trimethylcholest-22(E)-en-3 $\beta$-ol $\left(\mathrm{C}_{30} \Delta^{22}\right)$ and the 24(Z)-propylcholesta-5, 24(28)-dien-3 $\beta$-ol $\quad\left(\mathrm{C}_{30} \Delta^{5,24(28)}\right)$. In contrast to the marine samples, the freshwater sample from the Mackenzie River was dominated by $\mathrm{C}_{29} \Delta^{5}$ and $\mathrm{C}_{27} \Delta^{5}$.

The norC ${ }_{27} \Delta^{5,22}$ sterol was the most abundant sterol at sites 345-60, 235-70, 135-70 with percentage values higher than $20 \%$ of the total sterols.

In sediments, the sterol distribution was dominated by $\mathrm{C}_{29} \Delta^{5}, \mathrm{C}_{28} \Delta^{5,22}, \mathrm{C}_{27} \Delta^{5}$ and $\mathrm{C}_{28} \Delta^{5,24(28)}$. The $\alpha$-amyrin (Olean-12-en-3 $\alpha$-ol), a specific triterpenoid for angiosperms and also present in peat, was only measured in the sediments and its concentration was higher in the slope than in the shelf sediments.

\subsection{Fatty acids}

Concentrations of the total fatty acids and selected biomarkers in SPM and sediments are summarized in Tables 9 and 10.

Polyunsaturated fatty acids (PUFA) and short-chain fatty acids (SCFA) were the major FA classes in the SPM with values from 11 to $61 \%$ and from 25 to $77 \%$ of the total FAs, respectively. In sediments, the major component was the short-chain monounsaturated FA (SCMUFA (14-18) with percentages higher than $40 \%$, while PUFA and SCFA exhibited lower contributions with values ranging from 12 to $22 \%$ and from 21 to $26 \%$ of the total FAs, respectively. Typical molecular distributions are shown in Figs. 3c, 4 and 6 . At molecular level, marine suspended particles from the deep chlorophyll maximum of sites 130, 135, 235, 240, 345 and 460 were generally dominated by $\mathrm{C}_{16: 0}, \mathrm{C}_{14: 0}, \mathrm{C}_{18: 4 \omega 3}$, $\mathrm{C}_{18: 5 \omega 3}, \mathrm{C}_{22: 6 \omega 3}$ and $\mathrm{C}_{20: 5 \omega 3}$. In contrast, suspended particles of the freshwater site, the superficial water of site 130 (1303 ) and all the sediments exhibited distributions dominated by $\mathrm{C}_{16: 0}, \mathrm{C}_{16: 1 \omega 7}, \mathrm{C}_{14: 0}$ and $\mathrm{C}_{20: 5 \omega 3}$. Suspended particles of the deep chlorophyll maximum of sites 540 and 670 showed 
Table 5. Alcohol concentrations in the suspended particles from the Beaufort Sea $\left(\mathrm{ng} \mathrm{L}^{-1}\right)$. Percentage of total alcohols is given in brackets.

\begin{tabular}{|c|c|c|c|c|c|c|c|}
\hline $\begin{array}{l}\text { Locations- } \\
\text { Depth (m) }\end{array}$ & $\begin{array}{r}\mathrm{SCOH} \\
\left(n-\mathrm{C}_{14}-\mathrm{C}_{20}\right)\end{array}$ & $\begin{array}{r}\mathrm{LCOH} \\
\left(n-\mathrm{C}_{22}-\mathrm{C}_{30}\right)\end{array}$ & $n$-alcohols & $\begin{array}{l}\text { SCMUOH } \\
\left(\mathrm{C}_{14}-\mathrm{C}_{18}\right)\end{array}$ & $\begin{array}{l}\text { LCMUOH } \\
\left(\mathrm{C}_{20}-\mathrm{C}_{24}\right)\end{array}$ & $\begin{array}{r}\text { Phytol } \\
\text { compounds }\end{array}$ & $\begin{array}{r}\text { Total } \\
\text { alcohols }\end{array}$ \\
\hline $130-3$ & $4.4(7.2)$ & $24.5(40)$ & $28.9(47)$ & $1.6(2.6)$ & $5.9(9.7)$ & $25(40)$ & 61 \\
\hline $130-130$ & $4.0(25)$ & $0.0(0)$ & $4.0(25)$ & $1.2(7.5)$ & $3.8(24)$ & $6.7(43)$ & 16 \\
\hline $130-200$ & $1.0(9.2)$ & $0.0(0)$ & $1.0(9.2)$ & $1.0(8.9)$ & 4.1 (37) & $4.9(44)$ & 11 \\
\hline $135-40$ & $0.0(0.0)$ & $0.0(0)$ & $0.0(0)$ & $0.3(5.3)$ & $1.5(29)$ & $3.5(66)$ & 5 \\
\hline $135-70$ & $6.7(20)$ & $0.0(0)$ & $6.7(20)$ & $1.4(4.3)$ & 7.7 (23) & $17(52)$ & 33 \\
\hline $135-85$ & $0.6(3.8)$ & $0.0(0)$ & $0.6(4)$ & 0.5 (2.9) & $2.8(18)$ & $12(76)$ & 16 \\
\hline $135-145$ & $0.0(0.0)$ & $0.0(0)$ & $0.0(0)$ & $0.2(7.2)$ & 0.3 (14) & $1.9(79)$ & 2 \\
\hline $235-70$ & $4.7(24)$ & $0.0(0)$ & 4.7 (11) & $1.6(3.7)$ & $11.2(26)$ & $26(59)$ & 43 \\
\hline $235-85$ & $2.3(28)$ & $0.0(0)$ & $2.3(12)$ & $0.4(2.2)$ & $1.0(5.1)$ & $16(81)$ & 20 \\
\hline $235-145$ & $0.0(0.0)$ & $0.0(0)$ & $0.0(0)$ & $0.0(0)$ & $0.2(16)$ & $1.0(84)$ & 1 \\
\hline $235-200$ & $0.0(0.0)$ & $0.0(0)$ & $0.0(0)$ & $1.0(15)$ & $3.2(48)$ & $2.4(36)$ & 7 \\
\hline $240-70$ & $6.0(24)$ & $1.0(4.2)$ & $7.1(28)$ & $1.0(4.1)$ & $3.0(12)$ & $13.7(55)$ & 25 \\
\hline $240-130$ & $2.9(28)$ & $0.0(0)$ & $2.9(28)$ & $1.6(16)$ & $4.3(41)$ & $1.6(15)$ & 10 \\
\hline $240-200$ & $53(38)$ & $0.0(0)$ & $53(38)$ & $30(21)$ & $57(40)$ & $1.8(1.3)$ & 142 \\
\hline $345-60$ & $4.2(6.7)$ & $0.9(1.5)$ & $5.1(8.2)$ & $1.4(2.3)$ & $7.0(11)$ & $48(78)$ & 62 \\
\hline $345-85$ & $0.0(0.0)$ & $0.0(0)$ & $0.0(0)$ & $0.3(3.1)$ & $0.8(9)$ & 7.7 (88) & 9 \\
\hline $345-145$ & $0.0(0.0)$ & $0.0(0)$ & $0.0(0)$ & $0.0(0)$ & $0.3(38)$ & $0.5(63)$ & 1 \\
\hline $345-200$ & $1.5(26)$ & 0.7 (12.6) & $2.2(38)$ & $0.2(3.1)$ & $2.2(38)$ & $1.1(20)$ & 6 \\
\hline $460-70$ & $8.5(16)$ & $0.0(0)$ & $8.5(16)$ & $1.4(2.7)$ & $6.6(13)$ & $36(69)$ & 53 \\
\hline $460-130$ & $0.0(0.0)$ & $0.0(00$ & $0.0(0)$ & $0.2(9.3)$ & $0.3(16)$ & $1.4(75)$ & 2 \\
\hline $460-200$ & $0.0(0.0)$ & $0.0(0)$ & $0.0(0)$ & $0.0(0)$ & $0.2(8)$ & $2.6(92)$ & 3 \\
\hline $460-300$ & $6.4(41)$ & $0.0(0)$ & $6.4(41)$ & 2.9 (18) & $3.3(21)$ & 3.0 (19) & 16 \\
\hline $540-70$ & $6.8(31)$ & $0.0(0)$ & $6.8(30)$ & $1.4(6.1)$ & $4.0(18)$ & $10.1(45)$ & 22 \\
\hline $540-90$ & 1.7 (16) & $0.5(4.9)$ & $2.3(20)$ & $0.5(4.7)$ & $0.6(5)$ & $7.8(70)$ & 11 \\
\hline $540-200$ & $0.6(15)$ & $0.5(12.5)$ & $1.1(27)$ & $0.1(2.8)$ & $1.1(28)$ & $1.7(42)$ & 4 \\
\hline $640-70$ & $0.0(0.0)$ & $0.0(0)$ & $0.0(0)$ & $0.5(6.6)$ & $0.0(0)$ & $7.0(93)$ & 8 \\
\hline $640-130$ & $3.1(24)$ & $0.0(0)$ & $3.1(24)$ & $1.5(12)$ & $2.8(22)$ & $5.2(41)$ & 13 \\
\hline $640-200$ & $0.0(0.0)$ & $0.0(0)$ & $0.0(0)$ & $0.4(11)$ & $1.3(32)$ & $2.3(57)$ & 4 \\
\hline $760-130$ & $0.7(21)$ & $0.6(19.2)$ & $1.3(40)$ & $0.1(3.2)$ & $0.4(12)$ & $1.5(45)$ & 3 \\
\hline $760-200$ & $0.6(14)$ & $0.4(8.1)$ & $1.0(22)$ & $0.2(3.5)$ & $1.0(23)$ & $2.2(51)$ & 4 \\
\hline $760-300$ & $2.9(40)$ & 0.7 (9.9) & 3.7 (49) & $0.7(8.8)$ & $1.8(25)$ & $1.3(17)$ & 7 \\
\hline Mackenzie & 697 (27) & $886(35)$ & $1583(62)$ & $93(3.6)$ & $124(5)$ & 755 (30) & 2555 \\
\hline
\end{tabular}

Table 6. Alcohol concentrations in the sediments from the Beaufort Sea $\left(\mu \mathrm{g} \mathrm{g}^{-1}\right)$. Percentage of total alcohols is given in brackets.

\begin{tabular}{rrrrrrrrr}
\hline $\begin{array}{r}\text { Locations- } \\
\text { Depth }(\mathrm{m})\end{array}$ & $\begin{array}{r}\mathrm{SCOH} \\
\left(n-\mathrm{C}_{14}-\mathrm{C}_{20}\right)\end{array}$ & $\begin{array}{r}\mathrm{LCOH} \\
\left(n-\mathrm{C}_{22}-\mathrm{C}_{30}\right)\end{array}$ & $n$-alcohols & $\begin{array}{r}\text { SCMUOH } \\
\left(\mathrm{C}_{14}-\mathrm{C}_{18}\right)\end{array}$ & $\begin{array}{r}\text { LCMUOH } \\
\left(\mathrm{C}_{20}-\mathrm{C}_{24}\right)\end{array}$ & $\begin{array}{r}\text { Branched } \\
\text { alcohols }\end{array}$ & $\begin{array}{r}\text { Phytol } \\
\text { compounds }\end{array}$ & $\begin{array}{r}\text { Total } \\
\text { alcohols }\end{array}$ \\
\hline $390-47$ & $2.61(15)$ & $3.02(18)$ & $5.63(33)$ & $1.71(10)$ & $0.54(3)$ & $0.19(1)$ & $9.0(53)$ & 17.1 \\
$690-55$ & $1.29(11)$ & $3.12(27)$ & $4.41(38)$ & $1.57(13)$ & $0.37(3)$ & $0.09(1)$ & $5.3(45)$ & 11.7 \\
$260-60$ & $0.53(10)$ & $1.36(25)$ & $1.89(35)$ & $0.26(5)$ & $0.18(3)$ & $0.05(1)$ & $2.9(55)$ & 5.3 \\
$140-154$ & $1.26(16)$ & $1.86(23)$ & $3.13(39)$ & $0.95(12)$ & $0.34(4)$ & $0.04(0.5)$ & $3.5(44)$ & 8.0 \\
$235-576$ & $0.58(17)$ & $1.64(47)$ & $2.22(64)$ & $0.20(6)$ & $0.25(7)$ & $0.04(1)$ & $0.77(22)$ & 3.5 \\
$345-577$ & $0.74(16)$ & $2.23(48)$ & $2.97(64)$ & $0.20(4)$ & $0.40(9)$ & $0.05(1)$ & $0.99(21)$ & 4.6 \\
\hline
\end{tabular}


Table 7. Concentrations of sterols in suspended particulate matter (SPM) from the Beaufort Sea (ng $\mathrm{L}^{-1}$ ). Percentage of total sterols is given in brackets.

\begin{tabular}{|c|c|c|c|c|c|c|c|c|c|c|c|c|}
\hline $\begin{array}{l}\text { Locations- } \\
\text { Depth (m) }\end{array}$ & $\operatorname{norC}_{27} \Delta^{5,22}$ & $\mathrm{C}_{27} \Delta^{5,22}$ & $\mathrm{C}_{27} \Delta^{5}$ & $\mathrm{C}_{28} \Delta^{5,22}$ & $\mathrm{C}_{28} \Delta^{5,24(28)}$ & $\mathrm{C}_{28} \Delta^{5}$ & $\mathrm{C}_{29} \Delta^{5,22}$ & $\mathrm{C}_{29} \Delta^{5}$ & $\mathrm{C}_{29} \Delta^{5,24(28)}$ & $\mathrm{C}_{30} \Delta^{22}$ & $\mathrm{C}_{30} \Delta^{5,24(28)}$ & $\begin{array}{c}\text { Sum } \\
\text { sterols }\end{array}$ \\
\hline $130-3$ & $5.2(3)$ & $10.2(7)$ & $7.0(4)$ & $10.8(7)$ & $48.1(31)$ & $1.0(1)$ & $3.6(2)$ & $18.7(12)$ & $19.1(12)$ & $3.8(2)$ & $2.2(1)$ & 156 \\
\hline $130-130$ & $0.9(4)$ & $1.8(8)$ & 3.1 (14) & $2.4(10)$ & $2.1(9)$ & $0.4(2)$ & $1.1(5)$ & $2.8(12)$ & $1.7(7)$ & $1.0(5)$ & $0.5(2)$ & 22.8 \\
\hline $130-200$ & $0.8(4)$ & $1.6(7)$ & $2.6(12)$ & $3.6(16)$ & 2. (9) & $0.5(2)$ & $0.8(3)$ & $2.4(11)$ & $1.7(8)$ & $0.6(2)$ & $0.4(2)$ & 22.0 \\
\hline $135-40$ & $2.2(11)$ & $1.4(7)$ & $<2.4$ & $2.5(13)$ & $1.7(9)$ & $0.4(2)$ & $0.3(2)$ & $1.7(9)$ & $3.3(17)$ & $0.5(3)$ & $0.7(3)$ & 19.3 \\
\hline $135-70$ & $7.3(22)$ & $2.1(6)$ & $2.2(7)$ & 3.5 (11) & $2.9(9)$ & $0.6(2)$ & $0.7(2)$ & $2.4(7)$ & $3.5(11)$ & $0.5(1)$ & $1.5(4)$ & 32.9 \\
\hline $135-85$ & $2.7(12)$ & $1.3(6)$ & $1.9(9)$ & $2.2(10)$ & $2.1(10)$ & $0.7(3)$ & $0.7(3)$ & $1.7(8)$ & $3.4(16)$ & $0.2(1)$ & $0.8(4)$ & 21.7 \\
\hline $135-145$ & $0.3(4)$ & $0.6(9)$ & $<1.8$ & $0.8(11)$ & $0.9(13)$ & $0.4(6)$ & $0.3(5)$ & $1.0(14)$ & $0.6(9)$ & $0.1(1)$ & $0.1(2)$ & 7.09 \\
\hline $235-70$ & $10.9(28)$ & $2.3(6)$ & $2.2(9)$ & $5.1(13)$ & $3.1(8)$ & $0.8(2)$ & $0.7(2)$ & $2.4(6)$ & $4.0(10)$ & $0.5(1)$ & $1.1(3)$ & 39.5 \\
\hline $235-85$ & $3.3(17)$ & $1.2(6)$ & $<1.7$ & $2.2(11)$ & $2.3(12)$ & $1.2(6)$ & $0.6(3)$ & $1.6(8)$ & $2.9(15)$ & $0.2(1)$ & $0.9(5)$ & 19.6 \\
\hline $235-145$ & $0.4(6)$ & $0.6(9)$ & $<2.4$ & $1.0(14)$ & $0.5(7)$ & $0.03(0.4)$ & $0.4(6)$ & $1.6(23)$ & $0.8(12)$ & $0.1(1)$ & $0.1(2)$ & 6.96 \\
\hline $235-200$ & $0.8(6)$ & $1.3(9)$ & $<2.2$ & $2.3(16)$ & $1.2(9)$ & $0.5(3)$ & $0.7(5)$ & $2.2(15)$ & $1.5(10)$ & $0.3(2)$ & $0.3(2)$ & 14.5 \\
\hline $240-70$ & $2.5(10)$ & $2.3(10)$ & $2.2(9)$ & $2.7(11)$ & $3.3(14)$ & $0.8(3)$ & $0.4(2)$ & $1.7(7)$ & $3.0(13)$ & $0.1(0.3)$ & $1.0(4)$ & 23.8 \\
\hline $240-130$ & $0.7(4)$ & $1.4(9)$ & $3.2(19)$ & $1.7(10)$ & $1.0(6)$ & $0.4(2)$ & $0.8(5)$ & $2.4(14)$ & $1.2(8)$ & $0.3(2)$ & $0.2(1)$ & 16.4 \\
\hline $240-200$ & $0.7(4)$ & $1.6(9)$ & $2.3(14)$ & $2.1(13)$ & $1.2(7)$ & $0.4(2)$ & $0.6(4)$ & $1.9(11)$ & $1.5(9)$ & $0.3(2)$ & $0.3(2)$ & 16.4 \\
\hline $345-60$ & $11.3(26)$ & $2.3(5)$ & $2.4(6)$ & $5.8(13)$ & $3.6(8)$ & $0.6(2)$ & $0.7(2)$ & $2.1(5)$ & $5.2(12)$ & $0.5(1)$ & $2.1(5)$ & 43.2 \\
\hline $345-85$ & $0.8(9)$ & $0.7(8)$ & $<2.2$ & $1.2(14)$ & $1.2(13)$ & 0.07 (1) & $0.3(3)$ & $0.7(8)$ & $2.4(26)$ & $0.1(1)$ & $0.4(5)$ & 9.14 \\
\hline $345-145$ & $0.2(5)$ & $0.4(9)$ & $0.6(15)$ & 0.5 (13) & $0.3(6)$ & 0.03 (1) & $0.2(5)$ & $0.5(12)$ & $0.4(10)$ & 0.04 (1) & $0.1(3)$ & 4.00 \\
\hline $345-200$ & $0.5(4)$ & $0.9(7)$ & $1.8(15)$ & $1.6(13)$ & $0.8(7)$ & $0.4(3)$ & $0.6(5)$ & $1.5(13)$ & $1.2(10)$ & $0.2(2)$ & $0.3(2)$ & 11.9 \\
\hline $460-70$ & $5.8(13)$ & $2.5(6)$ & $2.3(5)$ & $5.2(12)$ & $4.4(10)$ & $0.4(1)$ & $0.9(2)$ & $2.7(6)$ & $8.8(20)$ & $0.4(1)$ & $3.5(8)$ & 43.5 \\
\hline $460-130$ & $0.3(5)$ & $0.5(10)$ & $<1.0$ & 0.9 (17) & $0.5(9)$ & 0.03 (1) & $0.3(5)$ & 0.7 (14) & 0.7 (12) & 0.1 (1) & $0.1(2)$ & 5.26 \\
\hline $460-200$ & $0.3(3)$ & $0.6(7)$ & $1.6(19)$ & $1.0(12)$ & $0.8(10)$ & $0.4(5)$ & $0.4(5)$ & $0.9(11)$ & $0.6(7)$ & $0.1(1)$ & $0.2(2)$ & 8.25 \\
\hline $460-300$ & $0.5(3)$ & $1.3(8)$ & $1.8(11)$ & 1.9 (12) & $1.7(10)$ & $1.1(7)$ & $0.6(4)$ & $1.9(12)$ & $1.2(7)$ & $0.3(2)$ & $0.2(1)$ & 16.4 \\
\hline $540-70$ & $1.1(6)$ & $1.1(6)$ & $1.4(8)$ & $1.3(8)$ & $1.9(11)$ & $0.8(5)$ & $0.6(4)$ & $3.1(18)$ & $2.2(13)$ & $0.1(0.3)$ & $0.9(5)$ & 17.4 \\
\hline $540-90$ & $1.2(12)$ & $0.7(8)$ & $1.0(10)$ & $1.7(18)$ & $0.7(7)$ & 0.1 (1) & $0.3(3)$ & $0.7(7)$ & $1.0(10)$ & $0.1(1)$ & $0.1(1)$ & 9.60 \\
\hline $540-200$ & $0.3(4)$ & $0.6(8)$ & $1.1(15)$ & $1.1(15)$ & $0.6(9)$ & $0.3(4)$ & $0.3(4)$ & $0.6(9)$ & $0.6(8)$ & $0.1(1)$ & $0.1(2)$ & 7.25 \\
\hline $640-70$ & $0.9(5)$ & $1.1(6)$ & $<7.7$ & $1.2(6)$ & $2.0(11)$ & $1.3(7)$ & $1.5(8)$ & $5.4(28)$ & $2.9(15)$ & $0.1(1)$ & $0.4(2)$ & 19.1 \\
\hline $640-130$ & $0.6(3)$ & $1.4(8)$ & $2.7(15)$ & $2.3(12)$ & $1.7(9)$ & $0.9(5)$ & 0.7 (4) & $2.2(12)$ & $1.4(8)$ & $0.3(1)$ & $0.3(1)$ & 18.1 \\
\hline $640-200$ & $0.4(3)$ & $0.9(8)$ & $1.2(11)$ & $1.4(13)$ & $1.2(11)$ & $0.5(5)$ & $0.5(4)$ & $1.2(11)$ & $1.1(10)$ & $0.3(3)$ & $0.2(2)$ & 10.9 \\
\hline $760-130$ & $0.3(4)$ & $0.5(7)$ & 0.9 (13) & $1.1(14)$ & $1.1(15)$ & $0.3(4)$ & $0.3(4)$ & $0.6(9)$ & $0.6(8)$ & $0.1(1)$ & $0.1(1)$ & 7.38 \\
\hline $760-200$ & $0.4(4)$ & $0.9(9)$ & $1.2(13)$ & $1.2(12)$ & $1.2(13)$ & $0.4(4)$ & $0.3(3)$ & $0.7(7)$ & $0.8(8)$ & $0.4(4)$ & $0.2(2)$ & 9.92 \\
\hline $760-300$ & $0.3(4)$ & $0.7(10)$ & $1.1(16)$ & $0.9(13)$ & $0.7(9)$ & $0.3(4)$ & $0.3(4)$ & $0.7(9)$ & $0.7(9)$ & $0.1(2)$ & $0.1(1)$ & 7.24 \\
\hline Mackenzie & $11.4(1)$ & $207(8)$ & $450(18)$ & $274(11)$ & $131(5)$ & $156(6)$ & $135(6)$ & $654(26)$ & $<1.27$ & $<1.27$ & $<1.27$ & 2473 \\
\hline
\end{tabular}

Table 8. Concentrations of sterols in sediments from the Beaufort Sea $\left(\mu \mathrm{g} \mathrm{g}^{-1}\right)$. Percentage of total sterols is given in brackets.

\begin{tabular}{|c|c|c|c|c|c|c|c|c|c|c|c|c|c|}
\hline $\begin{array}{l}\text { Locations- } \\
\text { Depth (m) }\end{array}$ & $\operatorname{norC}_{27} \Delta^{5,22}$ & $\mathrm{C}_{27} \Delta^{5,22}$ & $\mathrm{C}_{27} \Delta^{5}$ & $\mathrm{C}_{28} \Delta^{5,22}$ & $\mathrm{C}_{28} \Delta^{5,24(28)}$ & $\mathrm{C}_{28} \Delta^{5}$ & $\mathrm{C}_{29} \Delta^{5,22}$ & $\mathrm{C}_{29} \Delta^{5}$ & $\mathrm{C}_{29} \Delta^{5,24(28)}$ & $\mathrm{C}_{30} \Delta^{22}$ & $\mathrm{C}_{30} \Delta^{5,24(28)}$ & $\begin{array}{r}\text { Sum } \\
\text { sterols }\end{array}$ & $\begin{array}{r}\alpha \\
\text {-amyrin }\end{array}$ \\
\hline $390-47$ & $0.35(0.9)$ & $1.96(5)$ & 3.37 (9) & $5.02(14)$ & $3.64(10)$ & $3.71(10)$ & $2.42(7)$ & $8.81(24)$ & $1.88(5)$ & $0.25(1)$ & $1.31(3)$ & 36.8 & 0.044 \\
\hline $690-55$ & $0.18(1.2)$ & $1.01(6)$ & $2.03(13)$ & $1.04(7)$ & $2.23(14)$ & $1.15(7)$ & $0.52(3)$ & $2.42(15)$ & 0.64 (4) & $0.13(1)$ & $0.82(5)$ & 15.6 & 0.073 \\
\hline $260-60$ & $0.12(1.6)$ & $0.46(6)$ & $1.52(19)$ & $0.76(10)$ & $0.63(8)$ & 0.37 (5) & $0.31(4)$ & $1.11(14)$ & $0.41(5)$ & $0.15(2)$ & $0.18(2)$ & 7.80 & 0.080 \\
\hline $140-154$ & $0.23(1.9)$ & $0.87(7)$ & $1.58(13)$ & 1.99 (16) & $1.08(9)$ & $0.56(5)$ & $0.47(4)$ & $1.62(13)$ & $0.78(6)$ & $0.36(3)$ & 0.29 (2) & 12.2 & 0.104 \\
\hline $235-576$ & 0.09 (1.6) & $0.27(5)$ & $0.63(12)$ & $0.61(11)$ & $0.34(7)$ & 0.24 (4) & $0.27(5)$ & 1.06 (19) & $0.43(8)$ & 0.19 (3) & $0.16(3)$ & 5.43 & 0.077 \\
\hline $345-577$ & $0.19(2.0)$ & $0.58(6)$ & $0.98(10)$ & $1.11(12)$ & $0.61(6)$ & 0.34 (3) & 0.39 (4) & $1.99(21)$ & $0.92(10)$ & $0.27(3)$ & $0.32(3)$ & 9.42 & 0.086 \\
\hline
\end{tabular}

intermediate distributions between the two previously described, suggesting a mixed contribution of flagellates and diatoms at these sites.

Similar to the $\mathrm{LCOH}$, only the sediments, the superficial water of site 130 and the Mackenzie River sample contained the homologous series of long-chain $\mathrm{C}_{22}-\mathrm{C}_{30} n$-alkanoic acids (LCFA), typical of higher plants, with the $\mathrm{C}_{24}$ member being the most abundant.

The branched FA of known bacterial origin (BrFA), e.g. iso- and anteiso- $\mathrm{C}_{15}$ and $\mathrm{C}_{17}$ FAs, were minor components ( $<5 \%$ of total FAs). Highest concentrations were measured in the river sample and sediments, and the lowest values in the off-shore waters where they decreased from the DCM towards higher depths. At some sites (345, 240, 460, 640, 760 ), relatively high percentages were observed in the upper Pacific halocline at $\sim 130 \mathrm{~m}$ depth.

\subsection{Principal component analysis (PCA)}

Principal component analysis (PCA) was performed on a dataset from SPM consisting of weight ratios of individual fatty alcohols to total fatty alcohols, individual sterols to total sterols, and of FA groups and selected FA compounds to total FAs. The analysis was done with STATISTICA package for Windows (version 6.1). 
Table 9. Concentrations and diagnostic ratios of selected fatty acids (FAs) in SPM from the Beaufort Sea (ng L ${ }^{-1}$ ). Percentage of total FAs is given in brackets.

\begin{tabular}{|c|c|c|c|c|c|c|c|c|c|c|c|}
\hline $\begin{array}{l}\text { Locations- } \\
\text { Depth (m) }\end{array}$ & Total FAs & $\begin{array}{r}\text { SCFA } \\
\left(\mathrm{C}_{14}-\mathrm{C}_{20}\right) \\
\end{array}$ & $\begin{array}{r}\text { LCFA } \\
\left(\mathrm{C}_{22}-\mathrm{C}_{28}\right)\end{array}$ & $\begin{array}{r}\text { SCMUFA } \\
\left(\mathrm{C}_{14}-\mathrm{C}_{18}\right)\end{array}$ & $\begin{array}{l}\text { LCMUFA } \\
\left(\mathrm{C}_{20}-\mathrm{C}_{24}\right)\end{array}$ & $\mathrm{Br} F A$ & PUFA & $\mathrm{C}_{18}$ PUFA & $\mathrm{C}_{20: 5 \omega 3}$ & $\mathrm{C}_{22: 6 \omega 3}$ & $18: 1 \omega 9 / 18: 1 \omega 7$ \\
\hline $130-3$ & 2297 & 754 (33) & $47(2.1)$ & $496(22)$ & $7.2(0.3)$ & $29(1.3)$ & 907 (39) & $293(13)$ & $326(14)$ & $212(9)$ & 4.9 \\
\hline $130-130$ & 311 & $105(34)$ & $0.7(0.2)$ & $89(28)$ & $1.2(0.4)$ & $4.3(1.4)$ & $108(35)$ & 43 (14) & $25(8)$ & $31(10)$ & 9.0 \\
\hline $135-40$ & 266 & 104 (39) & $0.5(0.2)$ & $29(11)$ & $0.7(0.3)$ & $0.6(0.2)$ & $132(49)$ & $65(24)$ & $22(8)$ & $30(11)$ & 8.3 \\
\hline $135-70$ & 446 & $134(30)$ & $0.5(0.1)$ & $48(11)$ & $1.2(0.3)$ & $2.8(0.6)$ & $260(58)$ & $142(32)$ & $32(7)$ & $57(13)$ & 8.3 \\
\hline $135-85$ & 250 & $80(32)$ & $0.5(0.2)$ & $33(13)$ & $0.6(0.2)$ & $2.1(0.8)$ & $132(53)$ & $73(29)$ & $18(7)$ & $21(8)$ & 7.4 \\
\hline $235-70$ & 408 & $116(28)$ & $0.8(0.2)$ & $58(14)$ & $1.3(0.3)$ & $3.4(0.8)$ & $225(55)$ & $120(29)$ & $33(8)$ & $44(11)$ & 8.6 \\
\hline $235-85$ & 287 & $93(32)$ & $0.0(0.0)$ & $50(17)$ & $0.0(0.0)$ & $0.0(0.0)$ & $141(49)$ & $76(27)$ & $28(10)$ & $22(8)$ & 6.6 \\
\hline $235-145$ & 114 & $65(57)$ & $1.2(1)$ & $20(17)$ & $0.1(0.1)$ & $0.0(0.0)$ & $27(24)$ & $12(10)$ & $6.4(6)$ & $6.7(6)$ & 7.4 \\
\hline $235-200$ & 110 & $42(38)$ & $0.5(0.4)$ & $30(27)$ & $1.1(1)$ & $1.0(0.9)$ & $36(32)$ & $14(13)$ & $9.2(8)$ & $9.3(8)$ & 5.4 \\
\hline $240-70$ & 331 & $113(34)$ & $1.8(0.5)$ & $42(13)$ & $0.8(0.2)$ & $4.6(1.4)$ & $166(50)$ & $94(28)$ & $25(7)$ & $31(9)$ & 5.4 \\
\hline $240-130$ & 151 & $100(66)$ & $0.3(0.2)$ & $25(17)$ & $1.0(0.7)$ & $2.7(1.8)$ & $21(14)$ & $9.0(6)$ & $5.0(3)$ & $5.0(3)$ & 3.2 \\
\hline $345-200$ & 133 & $73(55)$ & $0.3(0.2)$ & $25(19)$ & $0.8(0.6)$ & $1.1(0.8)$ & $32(24)$ & $13(10)$ & $7.2(5)$ & $8.2(6)$ & 9.8 \\
\hline $460-70$ & 721 & $179(25)$ & $1.8(0.2)$ & 87 (12) & $1.1(0.2)$ & $3.7(0.5)$ & $444(61)$ & $246(34)$ & $63(9)$ & $86(12)$ & 6.5 \\
\hline $460-130$ & 68 & $28(41)$ & $0.1(0.1)$ & $15(21)$ & $0.0(0.0)$ & $0.8(1.1)$ & $24(35)$ & $9.4(14)$ & $6.2(9)$ & $6.2(9)$ & 5.1 \\
\hline $460-200$ & 252 & $106(42)$ & $0.7(0.3)$ & $73(29)$ & $0.5(0.2)$ & $1.1(0.4)$ & $67(26)$ & $16(6)$ & $30(12)$ & $15(6)$ & 6.8 \\
\hline $460-300$ & 286 & $97(34)$ & $0.4(0.1)$ & $101(35)$ & $4.1(1)$ & $2.2(0.8)$ & $80(28)$ & $15(5)$ & $40(14)$ & $20(7)$ & 4.3 \\
\hline $540-70$ & 315 & $81(26)$ & $0.9(0.3)$ & $71(22)$ & $0.8(0.3)$ & $1.6(0.5)$ & 155 (49) & 75 (24) & $40(13)$ & $25(8)$ & 8.0 \\
\hline $540-90$ & 175 & $45(26)$ & $0.5(0.3)$ & $34(20)$ & $0.2(0.1)$ & $1.2(0.7)$ & $92(52)$ & $55(31)$ & $12(7)$ & $15(9)$ & 11.0 \\
\hline $540-200$ & 129 & $44(34)$ & $0.5(0.4)$ & $37(29)$ & $0.5(0.4)$ & $1.3(1.0)$ & $44(34)$ & $18(14)$ & $12(9)$ & $10(8)$ & 8.1 \\
\hline $640-70$ & 922 & 716 (77) & $4.3(0.5)$ & $90(10)$ & $0.0(0.0)$ & $0.0(0.0)$ & $103(11)$ & $42(5)$ & $33(4)$ & $12(1)$ & 14.8 \\
\hline $640-130$ & 354 & $112(32)$ & $1.7(0.5)$ & $124(35)$ & $1.1(0.3)$ & $2.9(0.8)$ & $106(30)$ & $30(9)$ & $43(12)$ & $24(7)$ & 8.7 \\
\hline $640-200$ & 237 & $80(34)$ & $0.5(0.2)$ & $77(32)$ & $0.8(0.3)$ & $1.4(0.6)$ & $76(32)$ & 32 (14) & $24(10)$ & $14(6)$ & 7.1 \\
\hline
\end{tabular}

Table 10. Concentrations and diagnostic ratios of selected fatty acids (FAs) in sediments from the Beaufort Sea $\left(\mu g \mathrm{~g}^{-1}\right)$. Percentage of total FAs is given in brackets.

\begin{tabular}{rrrrrrrrrrrr}
\hline $\begin{array}{l}\text { Locations- } \\
\text { Depth }(\mathrm{m})\end{array}$ & Total FAs & $\begin{array}{r}\text { SCFA } \\
\left(\mathrm{C}_{14}-\mathrm{C}_{20}\right)\end{array}$ & $\begin{array}{r}\text { LCFA } \\
\left(\mathrm{C}_{22}-\mathrm{C}_{28}\right)\end{array}$ & $\begin{array}{r}\text { SCMUFA } \\
\left(\mathrm{C}_{14}-\mathrm{C}_{18}\right)\end{array}$ & $\begin{array}{r}\text { LCMUFA } \\
\left(\mathrm{C}_{20}-\mathrm{C}_{24}\right)\end{array}$ & Br FA & PUFA & $\mathrm{C}_{18}$ PUFA & $\mathrm{C}_{20: 5 \omega 3}$ & $\mathrm{C}_{22: 6 \omega 3}$ & $18: 1 \omega 9 / 18: 1 \omega 7$ \\
\hline $390-47$ & 572 & $147(26)$ & $6.2(1.1)$ & $281(49)$ & $2.6(0.5)$ & $6.9(1.2)$ & $125(22)$ & $13.8(2.4)$ & $75(13)$ & $6.1(1.1)$ & \\
$690-55$ & 250 & $55(22)$ & $5.1(2.0)$ & $131(52)$ & $1.0(0.4)$ & $3.1(1.2)$ & $53.7(21)$ & $8.91(3.6)$ & $32(13)$ & $2.8(1.1)$ & 1.0 \\
$260-60$ & 90.8 & $20(22)$ & $1.5(1.6)$ & $50.6(56)$ & $0.5(0.6)$ & $1.3(1.5)$ & $16.0(18)$ & $2.02(2.2)$ & $9.8(11)$ & $1.0(1.1)$ & 3.1 \\
$140-154$ & 252 & $53(21)$ & $2.3(0.9)$ & $153(61)$ & $0.8(0.3)$ & $2.3(0.9)$ & $38.4(15)$ & $5.27(2.3)$ & $23(9)$ & $2.6(1.0)$ & 0.4 \\
$235-576$ & 28.6 & $6.7(23)$ & $2.2(7.5)$ & $14.4(50)$ & $0.6(1.9)$ & $1.1(3.8)$ & $3.45(12)$ & $0.62(2.2)$ & $1.8(6)$ & $0.2(0.8)$ & 0.6 \\
$345-577$ & 30.3 & $7.4(25)$ & $2.5(8.2)$ & $12.5(41)$ & $0.7(2.2)$ & $1.5(5.0)$ & $5.37(18)$ & $0.76(2.5)$ & $2.6(8)$ & $0.5(1.6)$ & 0.5 \\
\hline
\end{tabular}

Figure 2 summarizes the PCA results. The plot (2A) distinguishes two groups which are characterized by typical factor loadings of their variables (2B). Phytol, norC $27 \Delta^{5,22}$ sterol, $\mathrm{C}_{18}$ PUFA and $\mathrm{C}_{22: 6 \omega 3}$, representing fresh phytoplankton dominated by flagellates and dinoflagellates, showed all negative factor loadings for $\mathrm{PC} 1$. The rest of sterols together with the short-chain monounsaturated fatty acids (SCMUFA), the branched FAs, the long-chain $n$-alcohols and FAs, indicating refractory material from both marine and terrestrial sources, showed all positive loadings. Similarly, the loadings for PC2 were positive for long-chain $n$-alcohols and $n$-fatty acids, branched FAs, LCMUOH $\left(\mathrm{C}_{20}-\mathrm{C}_{24}\right), \mathrm{C}_{16: 4 \omega 1}$ and phytanic acid, indicating zooplanktonic, terrestrial, diatom and bacterial sources. From these loadings the two clusters in the 
Table 11. Stable carbon isotopic composition $\left(\delta^{13} \mathrm{C}(\%\right.$ ) average of three replicate injections with S.D. $<0.8)$ of selected lipid biomarkers in SPM from Beaufort Sea.

\begin{tabular}{|c|c|c|c|c|c|c|c|c|c|c|c|}
\hline $\begin{array}{l}\text { Locations- } \\
\text { Depth (m) }\end{array}$ & $n-\mathrm{C}_{21: 6}$ & phytol & $\operatorname{norC}_{27} \Delta^{5,22}$ & $\mathrm{C}_{27} \Delta^{5}$ & $\mathrm{C} 28 \Delta^{5,24(28)}$ & $\mathrm{C} 29 \Delta^{5}$ & $\mathrm{C} 29 \Delta^{5,24(28)}$ & $i-\mathrm{C}_{15} \mathrm{FA}$ & $\mathrm{C}_{16} \mathrm{FA}$ & $\mathrm{C}_{20: 5 \omega 3}$ & $\mathrm{C}_{22: 6 \omega 3}$ \\
\hline $\begin{array}{r}130-3 \\
130-130 \\
130-200\end{array}$ & -32.9 & -30.0 & -32.3 & -29.8 & -26.4 & -32.5 & -32.9 & $\begin{array}{l}-24.5 \\
-24.0\end{array}$ & $\begin{array}{l}-30.4 \\
-28.6 \\
-29.2\end{array}$ & $\begin{array}{l}-31.9 \\
-32.4 \\
-32.6\end{array}$ & $\begin{array}{l}-32.3 \\
-31.8 \\
-32.9\end{array}$ \\
\hline $\begin{array}{r}135-40 \\
135-70 \\
135-85 \\
135-145\end{array}$ & & -36.1 & -28.1 & -28.6 & -31.8 & -31.7 & -33.2 & $\begin{array}{r}-29.3^{*} \\
-28.5 \\
-26.3\end{array}$ & $\begin{array}{l}-31.1 \\
-30.4 \\
-28.6\end{array}$ & $\begin{array}{l}-37.2 \\
-33.1 \\
-30.8\end{array}$ & $\begin{array}{l}-36.0 \\
-33.5 \\
-32.6\end{array}$ \\
\hline $\begin{array}{r}235-70 \\
235-145 \\
235-200\end{array}$ & & -40.6 & -33.5 & & -29.8 & $-32.1^{*}$ & -34.2 & $\begin{array}{l}-29.2 * \\
-27.0\end{array}$ & $\begin{array}{l}-30.7 \\
-27.7 \\
-29.3\end{array}$ & $\begin{array}{l}-33.2 \\
-33.5 \\
-32.7\end{array}$ & $\begin{array}{l}-34.8 \\
-32.6 \\
-32.7\end{array}$ \\
\hline $\begin{array}{r}240-70 \\
240-200\end{array}$ & $\begin{array}{l}-36.6 \\
-33.5\end{array}$ & -36.5 & -26.6 & -26.9 & $\begin{array}{l}-33.7 \\
-28.0\end{array}$ & $\begin{array}{l}-31.3 \\
-29.6\end{array}$ & $\begin{array}{r}-34.5 \\
-32.5^{*}\end{array}$ & -28.9 & $\begin{array}{l}-30.2 \\
-29.5\end{array}$ & $\begin{array}{l}-35.0 \\
-31.9\end{array}$ & $\begin{array}{l}-35.5 \\
-32.1\end{array}$ \\
\hline $\begin{array}{r}345-60 \\
345-85 \\
345-145 \\
345-200\end{array}$ & -35.6 & $\begin{array}{l}-30.9 \\
-37.9\end{array}$ & $\begin{array}{l}-23.6 \\
-27.9\end{array}$ & -27.3 & $\begin{array}{l}-32.0 \\
-34.0\end{array}$ & -31.9 & $\begin{array}{l}-33.9 \\
-36.1\end{array}$ & $\begin{array}{r}-27.4^{*} \\
-26.7\end{array}$ & -31.2 & $\begin{array}{l}-33.2 \\
-32.9 \\
-33.3\end{array}$ & $\begin{array}{l}-31.9 \\
-32.2 \\
-33.2\end{array}$ \\
\hline $\begin{array}{r}460-70 \\
460-300\end{array}$ & -37.2 & & & & & & & & $\begin{array}{l}-32.9 \\
-29.1\end{array}$ & $\begin{array}{l}-34.2 \\
-32.4\end{array}$ & $\begin{array}{l}-34.7 \\
-32.3\end{array}$ \\
\hline $\begin{array}{r}540-70 \\
540-200\end{array}$ & -36.1 & & & & & & & $\begin{array}{r}-27.0^{*} \\
-26.5\end{array}$ & $\begin{array}{l}-30.5 \\
-30.2\end{array}$ & $\begin{array}{l}-32.5 \\
-32.9\end{array}$ & $\begin{array}{l}-33.4 \\
-32.9\end{array}$ \\
\hline $\begin{array}{r}640-70 \\
640-200 \\
760-200\end{array}$ & & & & & & & & $\begin{array}{l}-26.2 \\
-29.1\end{array}$ & $\begin{array}{l}-29.4 \\
-31.1 \\
-29.7\end{array}$ & $\begin{array}{l}-30.6 \\
-32.9 \\
-32.8\end{array}$ & $\begin{array}{l}-32.4 \\
-32.7 \\
-32.9\end{array}$ \\
\hline Mackenzie & -35.2 & -35.9 & & -31.1 & -29.4 & -30.7 & & -29.6 & -31.0 & -36.0 & -34.5 \\
\hline
\end{tabular}

$*$ Values with $1<$ S.D. $<2$.

Table 12. Stable carbon isotopic composition $\left(\delta^{13} \mathrm{C}(\%)\right.$ average of three replicate injections with S.D. $\left.<0.8\right)$ of selected lipid biomarkers in sediments from Beaufort Sea.

\begin{tabular}{rrrrrrrrrrrrrr}
\hline $\begin{array}{c}\text { Locations- } \\
\text { Depth (m) }\end{array}$ & $\mathrm{C}_{23}$ & $\mathrm{C}_{29}$ & $\mathrm{IP}_{25}$ & $n-\mathrm{C}_{21: 6}$ & Phytol & $\mathrm{C}_{26}-\mathrm{OH}$ & $\mathrm{C}_{28} \Delta^{5,24(28)}$ & $\mathrm{C}_{29} \Delta^{5}$ & $i-\mathrm{C}_{15}$ & $\mathrm{C}_{16} \mathrm{FA}$ & $\mathrm{C}_{20: 5 \omega 3}$ & $\mathrm{C}_{26}-\mathrm{FA}$ \\
\hline $390-47$ & -30.1 & -30.2 & -20.6 & -29.5 & -27.4 & -31.2 & -24.3 & -24.0 & $-28.4^{*}$ & -28.2 & -29.6 & -30.5 \\
$690-55$ & -30.1 & -30.9 & & -31.5 & -29.1 & -31.5 & -25.5 & -28.6 & $-29.3^{*}$ & -31.1 & -31.8 & -32.1 \\
$260-60$ & -30.4 & -29.8 & -18.7 & -29.2 & -28.4 & -31.9 & -24.2 & -26.7 & -27.6 & -28.5 & -29.8 & $-30.7^{*}$ \\
$140-154$ & -29.8 & -30.3 & -17.5 & -31.5 & -27.9 & -30.5 & -24.6 & -27.2 & & -30.0 & -32.3 & -30.9 \\
$235-576$ & -30.2 & -30.3 & -19.9 & & -28.3 & -31.1 & $-25.4^{*}$ & -27.2 & $-27.5^{*}$ & -29.4 & -31.6 & $-30.6^{*}$ \\
$345-577$ & -29.7 & -30.9 & -20.3 & & -29.1 & -30.9 & -26.6 & -27.2 & -26.3 & -28.0 & -30.1 & -30.0 \\
\hline
\end{tabular}

* Values with $1<$ S.D. $<2$.

plot represent a group with high negative PC1 loadings (fresh phytoplankton component) and a group with a high positive PC1 contribution (marine and terrestrial refractory material). The first group represents suspended particles from the DCM (60-85 m), except for the station 640-70 and the second group all the deeper suspended particles $(\geq 100 \mathrm{~m})$ and the superficial 640-70. Other scattered and particular samples included the Mackenzie River, with high contribution of terrestrial and refractory material, the 240-200 with high contribution of zooplankton material, the 130-3 with some refractory and diatom material, and the 135-40 with very low phytoplankton marker concentrations.

\subsection{Compound-specific isotope analysis}

Tables 11 and 12 summarize the stable carbon isotopic composition $\delta^{13} \mathrm{C}(\% o)$ of selected lipid biomarkers in SPM and sediments. Values are given as mean of triplicate injections with s.d. Additional $\delta^{13} \mathrm{C}$ data are also displayed together with their molecular abundances in Figs. 3, 4, 6 and 9. 

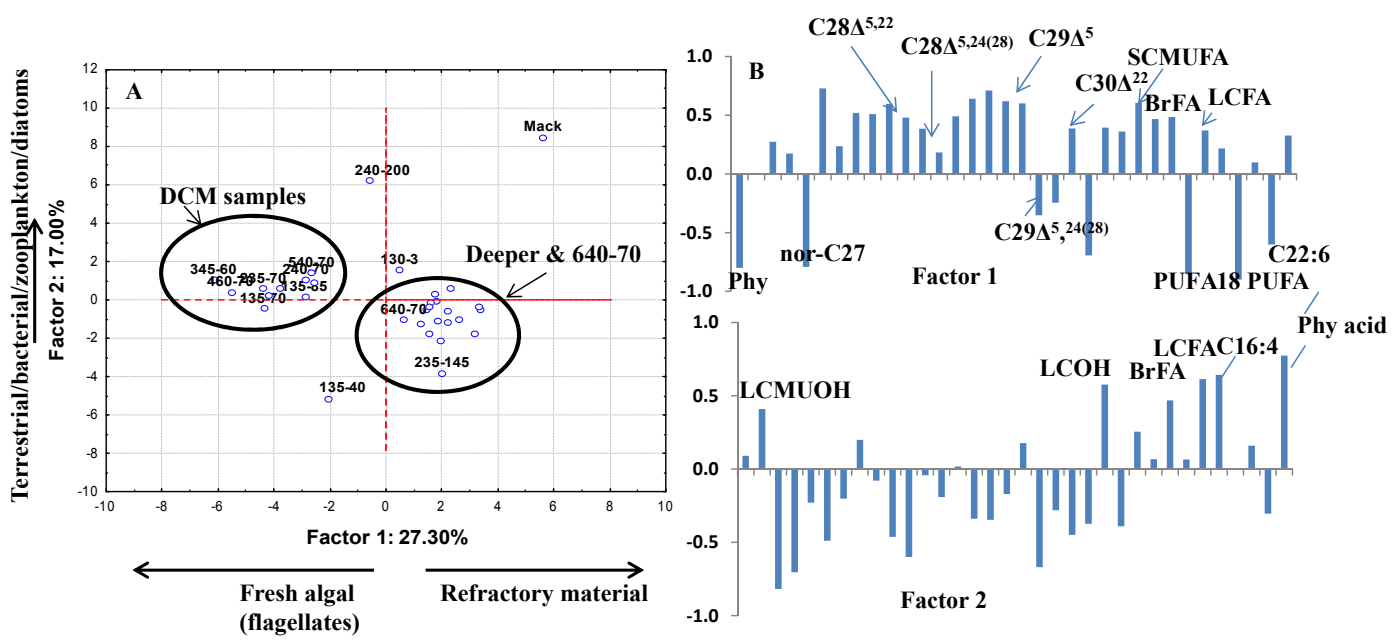

Fig. 2. Principal component score plot for each suspended particulate matter sample (A), showing $44 \%$ of the variance and (B) factor loadings for the variables.
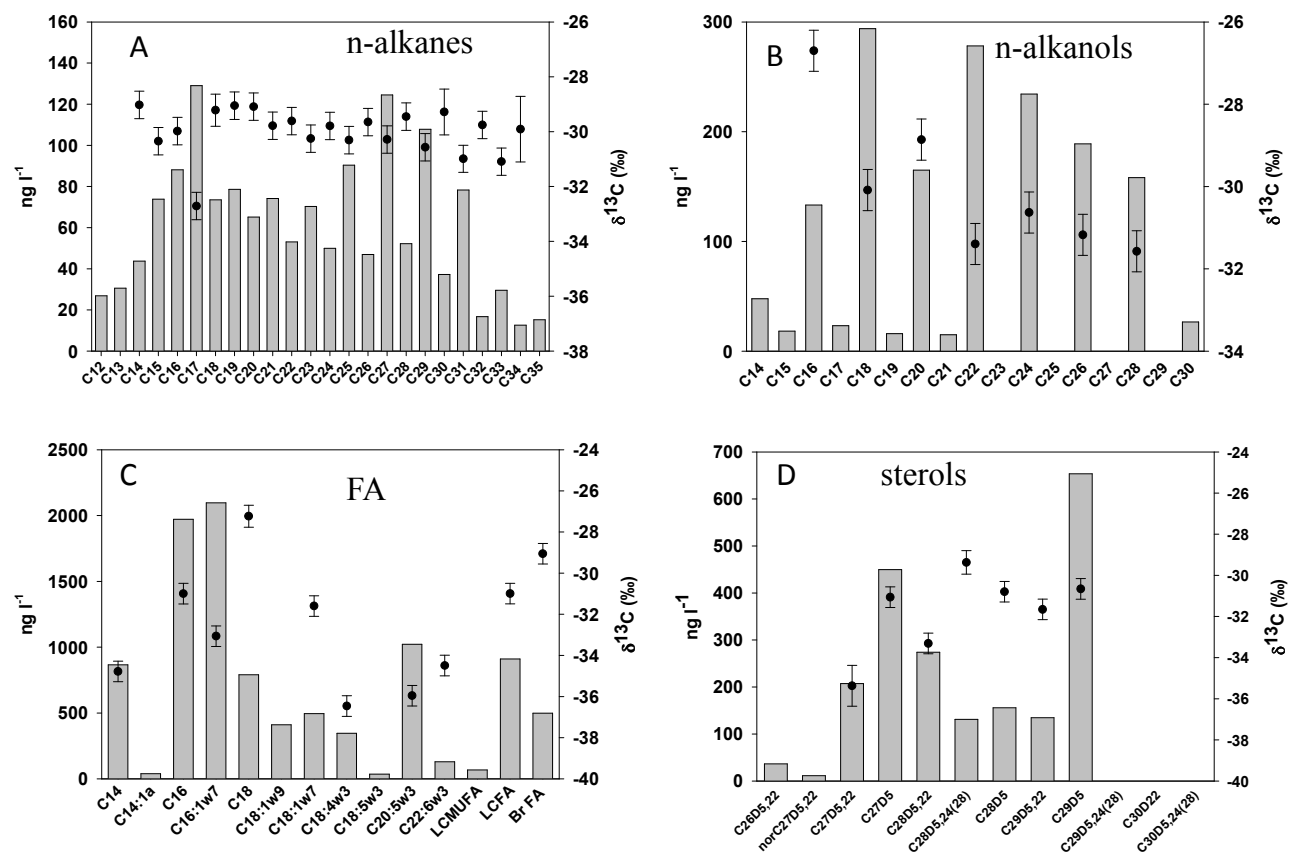

Fig. 3. Concentrations (bars) and carbon isotopic ratios (mean $\pm \mathrm{SD}, n=3$ ) of selected molecular biomarkers in the suspended particulate matter from the Mackenzie River: (A) $n$-alkanes; (B) $n$-alkanols; (C) selected fatty acid (FA) compounds; and (D) sterol compounds.

Carbon isotope ratios of phytol in SPM showed a large range of values, from $-31 \%$ at site $345-60$, where a bloom of flagellates/dinoflagellates occurred, to values as low as $-40 \%$ at site $235-70$, where dinoflagellates were predominant. At site 345, a $-7 \%$ o depletion between samples at 60 and $85 \mathrm{~m}$ was observed. The most depleted values were even more depleted than the one measured in the freshwater sample $(-36 \%)$. In contrast to the SPM, carbon isotope composition of phytol in sediments was quite homogeneous with values from -27 to $-29 \%$.
Isotope values of the algal planktonic $n-\mathrm{C}_{21: 6}$ in the marine SPM $(-33$ to $-37 \%$ o) were not different from the value obtained in the Mackenzie River $(-35 \%$ ) , and more enriched values were obtained in the sediment samples $(-29$ to $-31 \%$ o).

The $\delta^{13} \mathrm{C}$ signatures of the FAs in our SPM and sediments from the shelf are more depleted than those of previous studies (Goñi et al., 2000, 2005) that report values of -19 to $-24 \%$ using the methodology of alkaline $\mathrm{CuO}$. 

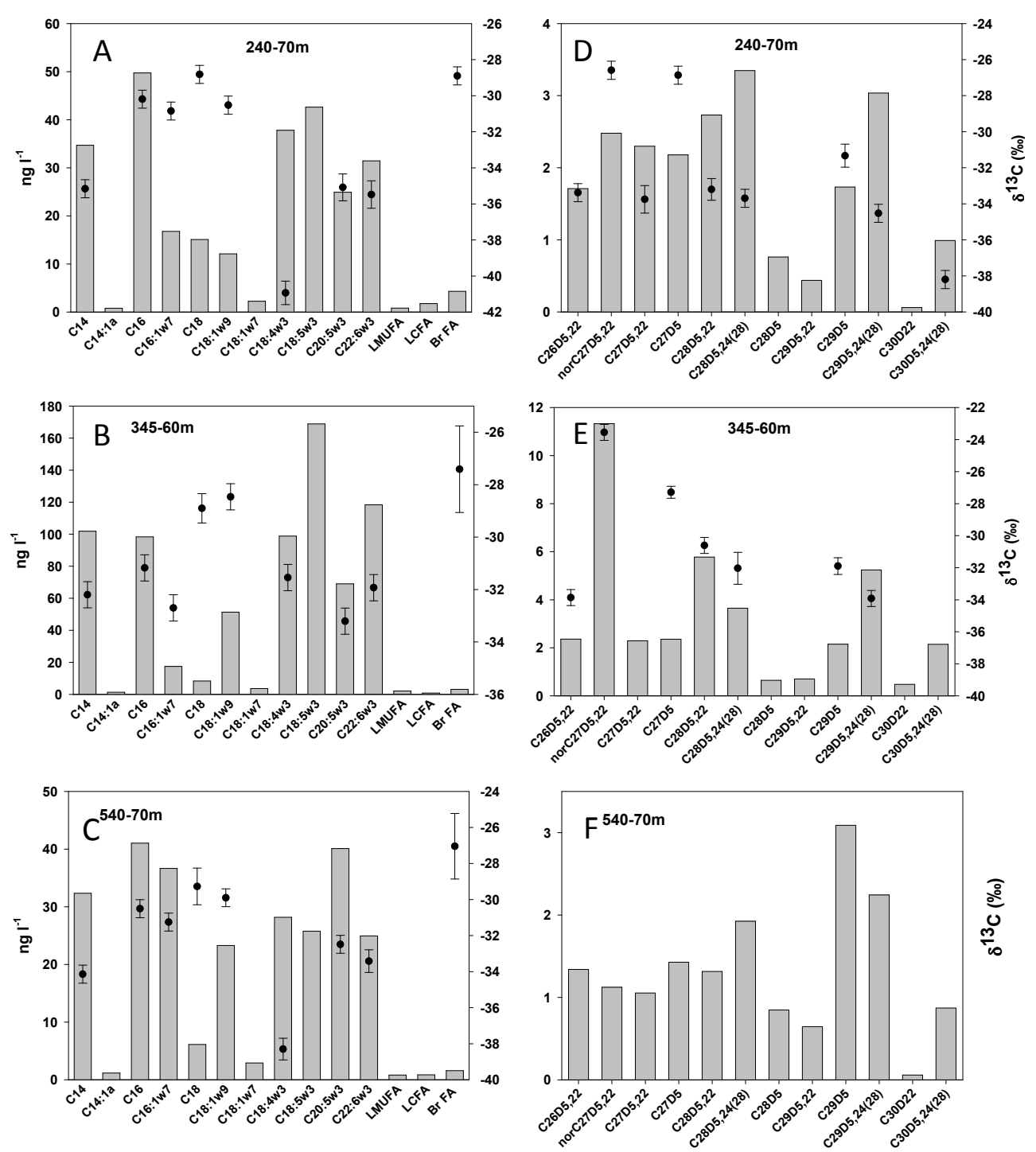

Fig. 4. Concentrations (bars) and carbon isotopic ratios (mean $\pm \mathrm{SD}, n=3$ ) of selected fatty acids (A-C) and sterols (D-F) in selected SPM from the Mackenzie slope.

The $\delta^{13} \mathrm{C}$ values for the $n$-alkanes in sediments averaged $-29.5 \%$. The $\mathrm{C}_{27}$ and $\mathrm{C}_{29}$ homologues were slightly lighter showing mean $\delta^{13} \mathrm{C}$ values of -30 and $-30.5 \%$, respectively, which fell into the high range of $\mathrm{C}_{3}$ vascular plants ( -29 to $-39 \%$; Collister et al., 1994; Chikaraishi and Naraoka, 2003; Bi et al., 2005). Similar $\delta^{13} \mathrm{C}$-depleted values were obtained for the odd mid-chain alkanes $\left(\mathrm{C}_{23}-\mathrm{C}_{25}\right)$, giving no evidence for input of $\mathrm{C}_{4}$ plants or aquatic plants (Chikaraishi and Naraoka, 2005).

Isotopic values of the marker for sea ice algae with 25 carbon atom skeleton (ice proxy, $\mathrm{IP}_{25}$ ) in sediments ranged between -17 and $-21 \%$.

\section{Discussion}

\subsection{Sources of organic carbon in the suspended particlesof the Mackenzie River}

The Mackenzie River flows through various types of land including Arctic tundra, boreal forest, peatland and mountains (Dyke and Brooks, 2000). The tundra is an ecosystem rather decoupled from the river system and is likely to make a small contribution to the total OM. In contrast, the boreal forest, mostly conifers, is certainly an important source of terrigenous organic matter of higher plant origin (Carrie et al., 2009; Solomon et al., 2000). The Mackenzie River is also the likely source of fossil material, such as coal fragments, to the Beaufort Sea (Dyke and Brooks, 2000; Yunker et al., 2011). 
As it is shown in Fig. 3a, the algae-related $n$ - $\mathrm{C}_{17}$ alkane predominates over the short-chain $n$-alkanes in the Mackenzie River giving evidence of the importance of freshwater algae or photosynthetical bacteria (Han and Calvin, 1969). Its $\delta^{13} \mathrm{C}$ value of $-32.7 \%$ contrasts with those measured for the even $n$-alkanes, which are fossil derived $\left(\delta^{13} \mathrm{C}\right.$ of $\sim-30 \%$ ). A higher abundance and lower $\delta^{13} \mathrm{C}$ values of the odd-chain alkanes compared to the even alkanes can also be observed. This highlights the terrestrial contribution of the odd $n$-alkanes $\left(n-\mathrm{C}_{27}, n-\mathrm{C}_{29}, n-\mathrm{C}_{31}\right.$, with $\delta^{13} \mathrm{C}$ values of $-31 \%$ ), overlapping the petroleum-derived alkanes. This latter fossil signature is enhanced by the presence of a considerable amount of UCM, typical of fossil/petrogenic sources (Table 3). A confirmation of the fossil source was also obtained by mass fragmentograms $(\mathrm{m} / z 191$, not presented here), which exhibited a series of extended $\mathrm{C}_{32}-\mathrm{C}_{35}$ hopanes characteristic of oil-derived hydrocarbons. The contribution of $n-\mathrm{C}_{23}$ and $n-\mathrm{C}_{25}$ homologues relative to the other longchained $n$-alkanes, with a $n-\mathrm{C}_{23}$ to $n-\mathrm{C}_{23}$ plus $n-\mathrm{C}_{29}$ ratio $\sim 0.4-0.5$, indicates an additional contribution of $\mathrm{C}_{3}$-lower plants, such as mosses. Similar ratios were reported for the Eurasian Arctic rivers, where Sphagnum-rich peats were the major source of OM (Vonk et al., 2008). The distribution of $n$-alkanols with even carbon (Fig. 3b) showed maximum values at $n-\mathrm{C}_{18}$ and $n-\mathrm{C}_{22}$, and a decreasing trend from $n-\mathrm{C}_{22}$ to $n-\mathrm{C}_{28}$. This distribution which was already found in SPM from the Mackenzie River in 1987 (Yunker et al., 1995) indicates a mixing of bacterial, algal and vascular land plants. The $\delta^{13} \mathrm{C}$ values for the alcohols $n-\mathrm{C}_{22}$ to $n-\mathrm{C}_{28}$ were around $-31 \%$, typical of gymnosperms $\mathrm{C}_{3}$ plants (Chikaraishi and Naraoka, 2003, 2007). More enriched values were obtained for the short-chain $n$-alkanols ( -26 to $-30 \%$ ), which are likely to derive from bacterial sources. As expected, the compound-specific isotope values for lipids are depleted by $\sim 5-6 \%$ compared to the $\delta^{13} \mathrm{C}$ reported for bulk samples of the Mackenzie River ( -26 to $-27 \%$ ) (Goñi et al., 2005). Although lignin and $\delta^{13} \mathrm{C}$ data indicated that the major source of terrigenous material in this area consists of non-woody, $\mathrm{C}_{3}$ angiosperm vascular plant vegetation derived from the tussock vegetation (sedges, cotton grass) (Goñi et al., 2000; Naidu et al., 2000), our relatively enriched $n$-alkanes and $n$-alkanols $\delta^{13} \mathrm{C}$ values suggest that they might be derived from gymnosperms. Angiosperms usually have long-chain $n$ alkyl compounds depleted in ${ }^{13} \mathrm{C}$ compared to gymnosperms, with $n$-alkane $\delta^{13} \mathrm{C}$ values of $-36 \%$ for angiosperms and $-31.6 \%$ for gymnosperms (Chikaraishi and Naraoka, 2003, 2007).

The fatty acid signature exhibited by the Mackenzie River (Fig. 3c) shows a typical profile of diatoms with relatively depleted $\delta^{13} \mathrm{C}$ values (-36\%o), typical of freshwater phytoplankton. In addition to this aquatic production, the terrestrial component represented by the LCFA $\left(n-\mathrm{C}_{22}\right.$ to $n$ - $\left.\mathrm{C}_{28}\right)$ with $\delta^{13} \mathrm{C}$ values of $-31 \%$ confirms again the $\mathrm{C}_{3}$ higher plant contribution. The bacterial lipids represented by the branched FAs and $\mathrm{C}_{18: 1 \omega 7}$ were among the less important groups of FAs, and the $18: 1 \omega 9 / 18: 1 \omega 7<1$ indicates a major bacteria-derived source for the $\mathrm{C}_{18: 1 \omega 7}$ in the river SPM.

Sterol distribution in suspended particles from the Mackenzie River (Fig. 3d) was dominated by $\mathrm{C}_{29} \Delta^{5}, \mathrm{C}_{27} \Delta^{5}$, $\mathrm{C}_{28} \Delta^{5,22}$ and $\mathrm{C}_{27} \Delta^{5,22}$. In the river water, it is likely that part of the sterols present are derived from higher plants or macrophytes, e.g. $\mathrm{C}_{28} \Delta^{5}, \mathrm{C}_{29} \Delta^{5}$ and $\mathrm{C}_{29} \Delta^{5,22}$ sterols, sitosterol $\left(\mathrm{C}_{29} \Delta^{5}\right)$ being by far the most abundant sterol. Their $\delta^{13} \mathrm{C}$ values ranging from -30.6 to $-31.6 \%$ suggest a $\mathrm{C}_{3}$ terrestrial source, but these values are not distinguishable from those of the diatom-derived $\mathrm{C}_{28} \Delta^{5,24(28)}$ $(-30 \%)$. In contrast, the other two diatom related sterols, $\mathrm{C}_{28} \Delta^{5,22}$ and $\mathrm{C}_{27} \Delta^{5,22}$, show more depleted $\delta^{13} \mathrm{C}$ values $(-34$ to $-36 \%$ ), typical of freshwater phytoplankton. Phytoplankton growing in fresh waters typically have depleted $\delta^{13} \mathrm{C}$ values $\left(-25-42 \%\right.$ o) in comparison to $\mathrm{C}_{3}$ land plants $(-20$ to $-32 \%$ ) (Boutton, 1991). However, carbon isotopic data in freshwater ecosystems are not always source-specific, because values for freshwater phytoplankton and terrestrial plants can overlap (Cloern et al., 2002). Volkman (1986) suggested that an evaluation of campesterol/stigmasterol $/ \beta$ sitosterol ratios is necessary in order to determine if these sterols are appropriate to be used as terrestrial biomarkers. For various higher plants, the relative abundance of these plant sterols has been found to be $1 /(0.5-1.3) /(11.5-31)$ (Nishimura, 1977). Hence, the main source of sitosterol in the river water seems to be from terrestrial emergent vascular plants.

Overall, the lipid composition of the Mackenzie River water was characterized by a major fossil component, important amounts of algal material dominated by diatoms and a terrestrial component mainly derived from the $\mathrm{C}_{3}$ emergent plants.

\subsection{Sources of organic matter in the suspended particles of the off-shore marine water column}

Total primary productivity in the Beaufort Sea usually ranges from 30 to $70 \mathrm{~g} \mathrm{C} \mathrm{m}^{-2} \mathrm{yr}^{-1}$, indicating oligotrophic conditions (Brugel et al., 2009; Carmack et al., 2006, 2004; Mundy et al., 2009). In summer, nutrients are rapidly drawn from the surface layer, resulting in the formation of a subsurface chlorophyll maximum at 25 to $30 \mathrm{~m}$ depth (Carmack et al., 2004). In our samples from summer 2009, Chl $a$ concentrations were generally low $\left(<1 \mathrm{mg} \mathrm{m}^{-3}\right)$ throughout the water column with a subsurface maximum centered around 60-70 m depth. The highest concentrations of marine algal biomarkers and $\mathrm{Chl} a$ were found on the east side of the main Mackenzie runoff (sites 345 and 460). Typical distributions of the diagnostic fatty acids and sterols are shown in Fig. 4. As it is observed in Fig. 4 and Table 9, the greater concentration of $\mathrm{C}_{22: 6 \omega 3}$ over $\mathrm{C}_{20: 5 \omega 3}$, together with the high concentrations of $\mathrm{C}_{18: 4 \omega 3}$ and $\mathrm{C}_{18: 5 \omega 3}$, in the upper $100 \mathrm{~m}$ of the eastern part of the study area (sites 130, 135, 235, 240, 345, 460) suggests that flagellates, including Prymnesiophyceae and Prasinophyceae (Dunstan et al., 1992), and 
dinoflagellates were the major constituents of the phytoplankton in the surface waters of this area. In contrast, the higher contribution of $\mathrm{C}_{20: 5 \omega 3}$ in the offshore waters from the western sector of the study area (sites 540, 640 and 760), and in the lower Atlantic halocline water ( $>200 \mathrm{~m}$ depth), of all sites sampled indicates a prevalence of diatom remnants. Moreover, the sterol profile dominated by $\mathrm{C}_{28} \Delta^{5,24(28)}$ and $\mathrm{C}_{29} \Delta^{5,24(28)}$ (Fig. 4d) may indicate that OM derives from prasinophytes (Volkman et al., 1994). These results are consistent with the findings of Balzano et al. (2012) and Lovejoy et al. (2007), where Arctic Micromonas, a typical Prasinophyceae was shown to be the dominant picoplankton during the sampling period. Previous studies (autumn 20032004) also showed higher abundances of prasinophytes in the Mackenzie Shelf contributing up to $38 \%$ of the total phytoplankton community (Brugel et al., 2009).

The most enriched $\delta^{13} \mathrm{C}$ values of phytol (Table 11) were measured at 3 and $60 \mathrm{~m}$ depths at sites 130 and 345, respectively, suggesting the highest growth rates. The large distribution of $\delta^{13} \mathrm{C}$ values (Table 11) accounts for differences in light regime and growth rate. These results are consistent with the large range in $\delta^{13} \mathrm{C}$ bulk values $(-27 \%$ o to $-18 \%$ ) displayed by biogenic particulate $\mathrm{OM}$ in the Arctic Ocean (Goericke and Fry, 1994). The $\delta^{13} \mathrm{C}$ values observed for the straight-chain polyunsaturated alkene $n-\mathrm{C}_{21: 6}$ ranged from -33 to $-37 \%$ o (Table 11) and are more depleted than those obtained in the sediment samples $(-29$ to $-31 \%$ ) and the value reported by Belt et al. (2008) in a sediment trap from the Franklin Bay $(-28 \%$ o). Although $\delta^{13} \mathrm{C}$ values of -35 to $-42.2 \%$ for polyunsaturated highly branched isoprenoid isomers from the Baffin Bay were suggested to result from freshwater diatoms (Belt et al., 2008), the so depleted values obtained in marine waters (sites 240, 345,460 and 540) suggest that the algal compounds (hydrocarbon $n-\mathrm{C}_{21: 6}$ and $\mathrm{C}_{20: 5 \omega 3}$ and $\mathrm{C}_{22: 6 \omega 3} \mathrm{FAs}$ ) are derived from slow-growing phytoplankton (Benthien et al., 2007). Indeed, the isotopic signature of pelagic phytoplankton from high latitudes, with relatively high concentrations of $\mathrm{CO}_{2}$, can be significantly depleted in ${ }^{13} \mathrm{C}$, with $\delta^{13} \mathrm{C}$ values ranging from -18 to $-28 \%$ in the Arctic Ocean (Goericke and Fry, 1994; Gradinger, 2009; Iken et al., 2005; Ruttenberg and Goñi, 1997; Schubert and Calvert, 2001; Tremblay et al., 2006). Also, the large isotopic offsets between algal biomass and eukaryotic lipid biomarkers ranging from -2 to $12 \%$ (Schouten et al., 1998; Hayes, 2001) are accounting for the relatively depleted $\delta^{13} \mathrm{C}$ of the biomarkers.

Alkenones typical of four genera of haptophyte algae of the class of Prymnesiophyceae belonging to the Isochrysidales order (Emiliania, Gephyrocapsa, Isochrysis and Chrysotila) were not detected in any of the water and sediment samples of the present study. This outcome is consistent with the taxonomic results, which report the absence of coccolithophorids (Coupel, personal communication, 2011). However, Chrysochromulina spp. belonging to the order of Prymnesiales was observed by Balzano et al. (2012), and sediment traps intercepted coccolithophorids in summer 2004 in Franklin Bay, west of the Amundsen Gulf (Forest et al., 2008). Generally, the occurrence of this class of algae in the Arctic Ocean and adjacent seas is very controversial. Coccolith production appears to be nearly absent in the North Water Polynya of the Baffin Bay (Hargrave et al., 2002), whereas large blooms of Emiliania huxleyi occur on the eastern Bering Shelf area (Murata, 2006). Moreover, alkenones found in surface sediments from the Laptev Sea were likely derived from coccolithophorids or other Prymnesiophyceae transported by Atlantic water masses along the continental slope (Fahl and Stein, 1997). All these findings might be related to the geochemical control on primary productivity. For instance, the silicate-rich Pacific waters versus the carbonate-rich Atlantic waters seem to potentially offer different advantages between diatoms/flagellates and coccolithophorids (Carmack et al., 2006).

As it can be observed in the first component of the PCA (Fig. 2b), the correlation of phytol with norC $27 \Delta^{5,22}$ was better than with the $\mathrm{C}_{28} \Delta^{5,22}, \mathrm{C}_{28} \Delta^{5,24(28)}$ diatom sterols (positive scores), showing that the phytoplankton biomass was dominated by dinoflagellates/flagellates. No correlation was found between dinosterol and $\operatorname{norC}_{27} \Delta^{5,22}$, which indicates that they derive from different organisms. Microscopy counts during the Malina cruise revealed the presence of several dinoflagellate species belonging to the genera Gymnodinium and Gyrodinium (Balzano et al., 2012). Overall, the specific composition of the particulate matter from off-shore waters indicating the importance of dinoflagellates and prasinophytes, together with the low phytoplankton biomarker concentrations and low $\delta^{13} \mathrm{C}$, suggests post-bloom conditions during the survey. The low $\delta^{13} \mathrm{C}$ (Fig. 4, Table 11) likely results from the reduced growth rates that favour the assimilation of the lighter isotope and also from the repleted concentrations of $\mathrm{CO}_{2}$ in the cold waters.

The traces of $n$-alkanes from $\mathrm{C}_{23}$ to $\mathrm{C}_{33}$ with no odd/even predominance $(\mathrm{CPI} \sim 1)$ in all samples, except the most superficial one at $3 \mathrm{~m}$ depth, indicate fossil- or microbially derived hydrocarbons (Grimalt et al., 1988; Volkman et al., 1980). However, as UCM was not detected, the fossil component in the marine suspended particles was certainly very low. The suspended particulate sample from the upper PML $(3 \mathrm{~m})$ of site 130 stands out among all other SPM samples. It contains biogenic material dominated by diatoms plus terrigenous material (wax $n$-alkanes, LCOH, LCFA). A likely explanation for the presence of this material could be the deposition of aerosols on the ice. Plant waxes readily form aerosols and are subject to atmospheric transport. This together with ice melting and subsequent release of particles results in the transfer of terrigenous material from the shelf and land to the offshore waters (Pfirman et al., 1995). The apparent absence of terrestrial biomarkers in the rest of the suspended particles likely results from a massive dilution by marine-derived biomarkers. These observations are consistent with previous studies with sediment traps in the area, 
where the levels of terrigenous flux decreased substantially in summertime (Forest et al., 2007).

Moderate amounts of the LCMUOH fatty alcohols, typical of zooplankton, occurred in the deeper SPM, and in particular at site 240. Moreover, below the euphotic zone of site $240, \delta^{13} \mathrm{C}$ values indicated phytoplankton produced at higher growth rates at depth than at the DCM. Growth rates and $\delta^{13} \mathrm{C}$ values of compounds synthesized before and after a bloom are lower than those of compounds synthesized in the main exponential growth phase. All these parameters point out that post-bloom conditions prevailed in the euphotic layer of site 240, whereas the important signal of zooplankton and diatom markers below the euphotic layer gives evidence of the opportunistic behaviour of predators grazing on the sinking flux of remnants of phytoplankton produced during bloom conditions in the euphotic layer. Herbivorous grazers, particularly copepods, may at times effectively graze on suspended algal material in the slope of the Mackenzie Shelf (Forest et al., 2007).

The bacterial lipids represented by the branched FAs were among the less important groups of FAs, exhibiting the highest abundance in the DCM and decreasing from DCM to deep waters. However, percentage values were highest at $\sim 130 \mathrm{~m}$ depth of most sites $(240,345,460,640,760)$, corresponding to waters from the upper Pacific halocline. These results are consistent with those from Ortega-Retuerta (2012), where specific bacterial populations predominated in waters below $100 \mathrm{~m}$, and bacteria abundance decreased from river to offshore waters and from surface to deep waters. Although vaccenic acid $\left(\mathrm{C}_{18: 1 \omega 7}\right)$ is considered to be an indicator of bacterial input, its 18:1 $\omega 9 / 18: 1 \omega 7$ isomer ratio $>1$ (Table 9) suggests a dominant phytoplanktonic source in the marine offshore SPM, in contrast to the major bacteria-derived source in the river SPM.

The $\delta^{13} \mathrm{C}$ of sitosterol in off-shore SPM had similar depleted values as sitosterol in the river. But they were also similar to other algal sterols (Table 11), which makes it difficult to discern the source of sitosterol only by the $\delta^{13} \mathrm{C}$ value. However, the poor correlation between the concentrations of sitosterol and other phytoplanktonic markers (phytol, 24-methylenecholesterol, isofucosterol) (excluding the sample $130-3 \mathrm{~m})(r=0.29$ to 0.35$)$ seems to indicate the mixing of terrestrially and marine-derived sitosterol. Therefore, the terrestrial input derived from this biomarker in the Arctic Sea should be considered with caution. Still, the high percentages of sitosterol relative to total sterols at stations 540 and 640 point out the influence of the Mackenzie River plume. During Malina, the river plume extended farther offshore in the western channel than in the eastern channel, which was reflected by the spatial distribution of high CDOM absorption in the upper PML (Matsuoka et al., 2012).

In order to evaluate the quality and nature of the lipidic component of the OM, we estimated the relative contributions of $\mathrm{OM}$ constituents by grouping the different molecular lipid biomarkers into the following components: fossil (UCM, and petroleum hydrocarbons), fresh/labile algal (PUFA, phytol, $\mathrm{IP}_{25}, n-\mathrm{C}_{21: 6}, \mathrm{C}_{28} \Delta^{5,24(28)}$, $\mathrm{C}_{29} \Delta^{5,24(28)}$ ), refractory/detrital algal (SCFA, SCMUFA, rest of sterols, biogenic alkanes, SCOH, SCMUOH), zooplankton (LCMUFA, LCMUOH), bacterial (branched FAs) and $\mathrm{C}_{3}$ terrestrial plants (LCFA, LCOH, wax $n$-alkanes). Figure 5 shows the relative composition for each SPM sample including the freshwater sample from the Mackenzie River. For the Mackenzie River, $\mathrm{C}_{18: 1 \omega 7}$ was included within the bacterial and $\mathrm{C}_{29} \Delta^{5}$ within the terrestrial component. The fossil and refractory algal contributions are the dominant fractions of the freshwater OM from the river with $\sim 34 \%$ each of the total molecular lipid biomarkers, whereas the terrestrial contribution derived from the $\mathrm{C}_{3}$ vascular plants accounted for only $10 \%$ and the bacterial and zooplankton pools hardly reached $4 \%$. In contrast to the freshwater sample, the major contribution in the offshore SPM was from fresh and refractory algal components. As expected, the fresh/labile algal component dominated in the samples from the DCM, whereas the refractory algal material increased with water column depth. Heterotrophic bacterial biomarkers were relatively low in both marine and river samples. For comparison, the POC in samples collected with traps deployed at $\sim 100 \mathrm{~m}$ depth in the Mackenzie Shelf and slope in 2003-2004 was found to be predominantly marine, whereas the allochthonous (terrestrial) carbon contribution accounted for only 14 to $38 \%$ of total POC (Magen et al., 2010). Other studies based on the same approach of POC/Al ratio used by Magen et al. (2010) found that carbon fluxes of marine origin were higher than $80 \%$ in sediment traps deployed over the slope during summer 2004 (Forest et al., 2007) and averaged $70 \%$ of the total annual POC flux on the shelf during 1987-1988 (O’Brien et al., 2006). In contrast, using the two-end-member mixing model of the marine and terrestrial $\delta^{13} \mathrm{C}$ values, marine POC fluxes were as low as $\sim 10-15 \%$ of the terrestrial fluxes in the Mackenzie outer shelf (Amiel and Cochran, 2008). However, this percentage is extremely sensitive to the $\delta^{13} \mathrm{C}$ of the marine end-member. A decrease of one per mill yields an increase of approximately $20 \%$ in the estimated marine component.

\subsection{Sources of organic matter in the sediments}

All concentrations of biomarkers and total organic carbon (TOC) in surface sediments decreased from the shelf to the slope. Relatively high concentrations of phytol and PUFA, such as $\mathrm{C}_{20: 5 \omega 3}$ and $\mathrm{C}_{22: 6 \omega 3}$ in the sediments (Fig. 6 and Tables 6 and 10), indicate a contribution of fresh OM, which is likely due to a high rate of algal carbon flux, as these PUFA are quickly degraded in the water column after cells lyse. Since diatoms did not represent the major phytoplankton community in our water column samples, the abundance of $\mathrm{C}_{20: 5 \omega 3}$ in sediments reflects a previous dominance of diatoms in the water column. Their enriched $\delta^{13} \mathrm{C}$ values in sediments compared to those in SPM (Tables 11 and 12) 


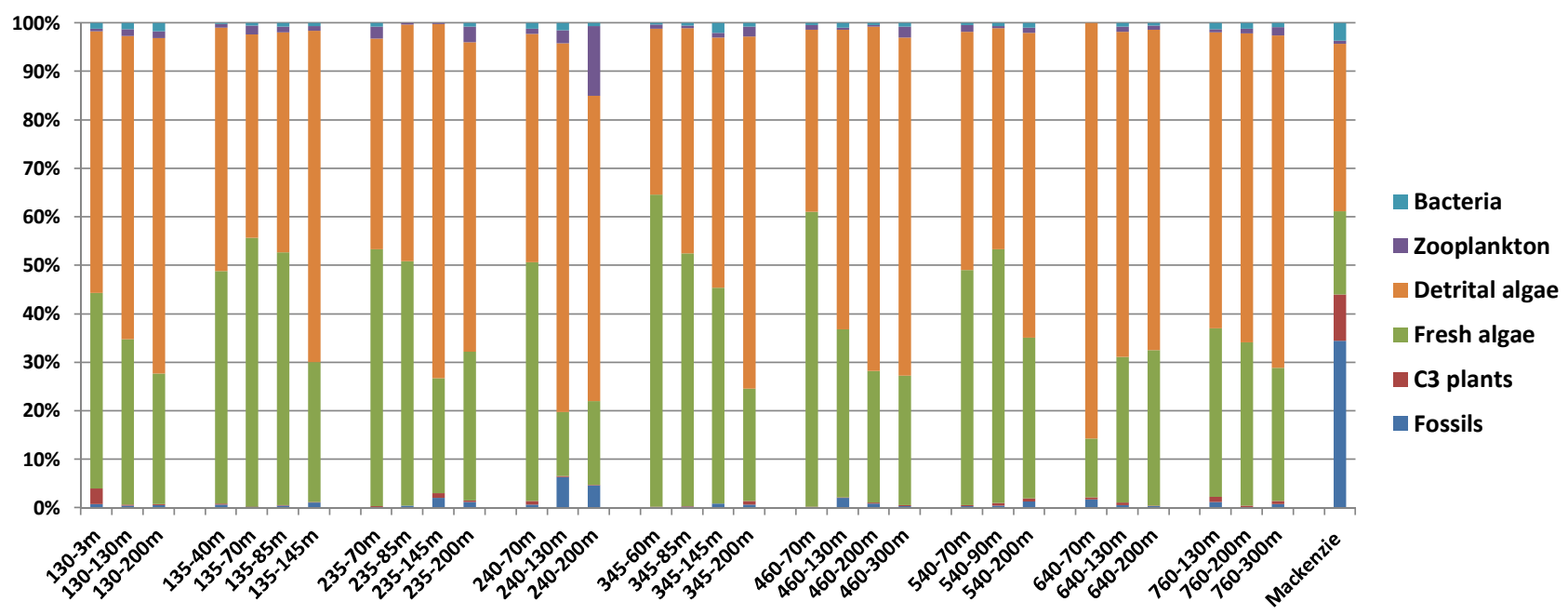

Fig. 5. Relative contributions of OM constituents in the SPM from the Mackenzie River and in the offshore waters from the Mackenzie slope. The molecular biomarkers were separated into the following components: fossil (UCM, and petroleum hydrocarbons), fresh/labile algal (PUFA, phytol, $\mathrm{IP}_{25}, n-\mathrm{C}_{21: 6}, \mathrm{C}_{28} \Delta^{5,24(28)}, \mathrm{C}_{29} \Delta^{5,24(28)}$ ), refractory/detrital algal (SCFA, SCMUFA, rest of sterols, biogenic alkanes, $\mathrm{SCOH}, \mathrm{SCMUOH}$ ), zooplankton (LCMUFA, LCMUOH), bacterial (branched FA) and $\mathrm{C}_{3}$ terrestrial plants (LCFA, LCOH, wax $n$-alkanes). For the Mackenzie River, $\mathrm{C}_{18: 1 \omega 7} \mathrm{FA}$ and $\mathrm{C}_{29} \Delta 5$ were included within respectively the bacterial and terrestrial component.

suggest the sinking of ungrazed diatoms derived from a bloom and/or from ice algae mats released when ice melts. A previous study in the area highlighted that diatoms were important primary producers during the summer, whereas in autumn smaller cells (e.g. flagellates) were dominant (Morata et al., 2008).

The presence of the $\mathrm{IP}_{25}$ with highly enriched $\delta^{13} \mathrm{C}$ values (Table 12) also confirms the input from ice algae in the sediments. These heavy $\delta^{13} \mathrm{C}$ values are well comparable with the data of Belt et al. (2008) from sediments of the Franklin Bay, and are distinguishable from those of OM of planktonic origin (e.g. phytol). Their percentage contribution to total algal hydrocarbons increased from the shelf $(5 \%)$ to offshore (48\%) (Fig. 7). Previous studies in this area estimated the production by ice algae to be between 2 and $30 \%$ of the total marine production (O'Brien et al., 2006, and references therein).

Sediment at site 390 showed the highest concentration of lipid biomarkers including the $\mathrm{IP}_{25}$ (Table 4), and exhibited the most enriched $\delta^{13} \mathrm{C}$ values for all sterols including sitosterol (with values of $\sim-24 \%$ ) (Table 12). These $\delta^{13} \mathrm{C}$ values are indicative of marine sources and, together with the distribution of FAs and sterols (Fig. 6), suggest that diatoms (including sea ice diatoms) can be the source of sitosterol at this site. Benthic microalgal production is believed to be small, and the production by macrophytes is not important in the Mackenzie Shelf (Macdonald et al., 1998). We rather believe that these results reflect the annual history of sea ice melting. This means that site 390 was likely to be situated within the flaw polynya (see satellite pictures of the ice cover in Forest et al., 2012) and was subject to a bloom early in the year, which explains the high concentrations of marine and ice algal biomarkers.

Heterotrophic bacteria play an important role in the degradation of the OM during early diagenesis. Branched FAs derived from bacterial biomass were more abundant in the nearshore sediments, and their $\delta^{13} \mathrm{C}$ values $\left(i-\mathrm{C}_{15}\right.$, Table 12) were relatively more enriched than those from terrestrial sources $\left(\mathrm{C}_{26} \mathrm{FA}\right)$ suggesting a predominant utilization of marine-derived OM. The $18: 1 \omega 9 / 18: 1 \omega 7$ ratios were $<1$ (Table 10) in all offshore and deeper sediments indicating the predominant bacterial origin of the $\mathrm{C}_{18: 1 \omega 7} \mathrm{FA}$ in these sediments. All these autochthonous natural inputs overlap with the allochthonous inputs derived from land plants and fossil material. The $\delta^{13} \mathrm{C}$ values for the $n$-alkanes, typical of $\mathrm{C}_{3}$ vascular plants, were consistent with those previously reported in surface sediments from the Beaufort Sea (Drenzek et al., 2007) and the Franklin Bay (Belt et al., 2008). Higher abundance of terrestrial LCOH and LCFA was measured in the shallow sediments (Tables 6 and 10), but the percentage of terrestrial material was higher in sediments underlying a deeper water column because of the higher lability of marine organic matter. Fossil material was evidenced by the moderate unresolved complex mixture (UCM) and the background of petrogenic $n$-alkanes. A low biodegradation of petroleumrelated inputs in this area was confirmed by the low ratio of $\mathrm{UCM} / n$-alkanes $(<7)$ (Table 4$)$, since values $>10$ are indicative of chronic/degraded petroleum contamination (Simoneit, 1982).

The correlation found between the long-chain $n$-alkanols, $n$-alkanoic acids and $n$-alkanes $(r>0.95)$ confirms that these three classes of terrigenous biomarkers have a common 

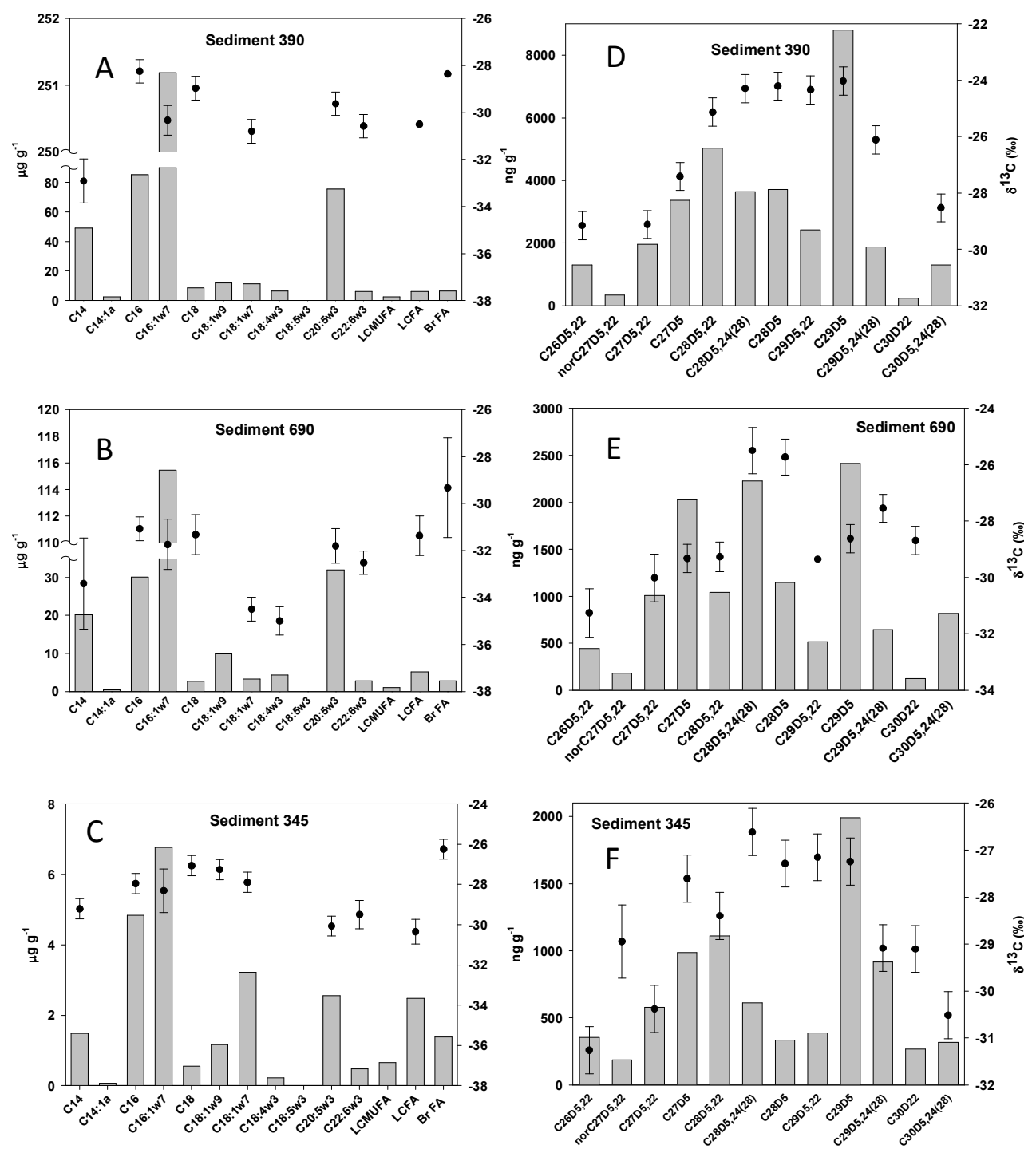

Fig. 6. Concentrations (bars) and carbon isotopic ratios (mean $\pm \mathrm{SD}, n=3$ ) of selected fatty acids (A-C) and sterols (D-F) in selected sediments from the Mackenzie Shelf and slope.

source, similar transport, deposition and degradation pathway in the area. Other common terrigenous biomarkers, such as $\alpha$-amyrin, retene and sitosterol exhibit relatively poor correlations with the long-chain terrestrial biomarkers. However, when site 390 is excluded, all other sediment samples showed relatively good correlation between sitosterol and long-chain terrestrial biomarkers, confirming the dominant terrestrial origin of sitosterol in these sediments. Also, the $\delta^{13} \mathrm{C}$ value of sitosterol in the sediment $390,-24 \%$, typical of marine sources contrasts with the more depleted values of the rest of sediments $(\sim 28 \%$ ) and that of the Mackenzie River $(-31 \%$ ) . Assuming the isotope ratio of the Mackenzie River $(-31 \%$ ) as the terrestrial and the value from sediment at site $390(-24 \%)$ as marine end-member, we estimate that $66 \%$ of sitosterol in sediments of the site 690 is terrestrial, whereas it was only $39 \%$ at the site 260 , and $45 \%$ in the slope and deeper sediments.

We estimated the relative contribution of the different $\mathrm{OM}$ components in the same way as in the SPM (Fig. 8). However, in sediments we included $\mathrm{C}_{18: 1 \omega 7}$ within the bacterial markers, sitosterol within the $\mathrm{C}_{3}$ terrestrial plant markers (except at site 390) and cholesterol within the zooplankton markers. It can be observed that in all sediments the algal component dominates over the fossil and $\mathrm{C}_{3}$ plant material. In particular, the sediments from the shelf and Amundsen Gulf present the highest contribution of detrital algal material (60-73\%), whereas the slope sediments (235 and $345)$ contain a higher proportion of fossil $(40 \%)$ and $C_{3}$ terrestrial plants $(10 \%)$. The contribution of zooplankton and bacteria-derived organic material is always minor and hardly 
\%IP25 over total algal hydrocarbons

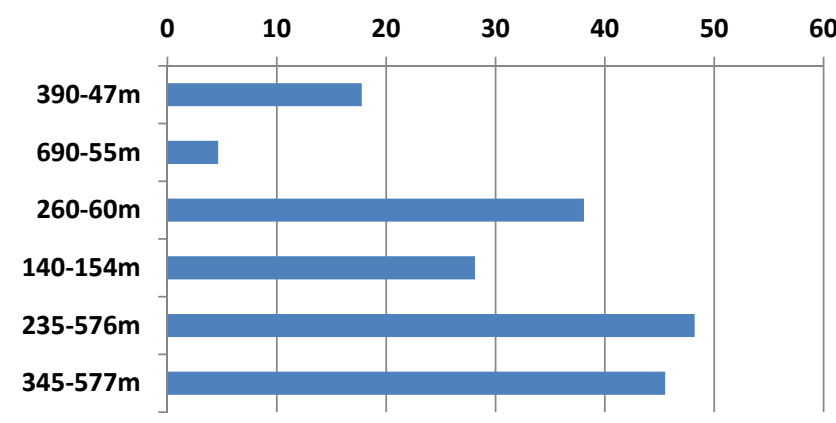

Fig. 7. Percentage contribution of the $\mathrm{C}_{25}$ monounsaturated hydrocarbon (sea ice $\mathrm{IP}_{25}$ biomarker) to the total algal hydrocarbons in sediments from the Mackenzie Shelf and slope. Total algal hydrocarbons include the $\mathrm{IP}_{25} n-\mathrm{C}_{15}, n-\mathrm{C}_{17}, n-\mathrm{C}_{19}, n-\mathrm{C}_{21: 6}, n-\mathrm{C}_{21: 5}$ and $n-\mathrm{C}_{21: 4}$.

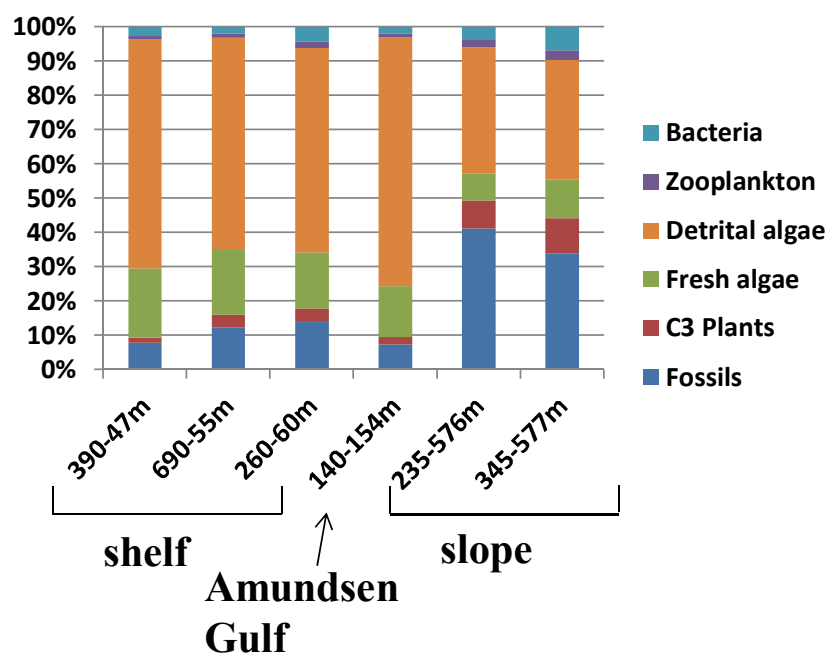

Fig. 8. Relative contributions of $\mathrm{OM}$ constituents in the sediments from the Mackenzie Shelf, Amundsen Gulf and slope. The molecular biomarkers were separated into the following components: fossil (UCM, and petroleum hydrocarbons), fresh/labile algal (PUFA, phytol, $\mathrm{IP}_{25}, n-\mathrm{C}_{21: 6}, \mathrm{C}_{28} \Delta^{5,24(28)}, \mathrm{C}_{29} \Delta^{5,24(28)}$ ), refractory/detrital algal (SCFA, SCMUFA, rest of sterols, biogenic alkanes, SCOH, SCMUOH), zooplankton (LCMUFA, LCMUOH and cholesterol), bacterial (branched FA, branched alcohols and $\mathrm{C}_{18: 1 \omega 7} \mathrm{FA}$ ) and $\mathrm{C}_{3}$ terrestrial plants (LCFA, LCOH, wax $n$-alkanes and sitosterol (except for site 390)).

reaches respectively $3 \%$ and $7 \%$ of the total quantified lipid molecular biomarkers. If we consider that the fossil and $\mathrm{C}_{3}$ plant material represent the total allochthonous lipidic component, then autochthonous sources contributed more than allochthonous sources to the OM lipid pool in the sediments of the shelf and Amundsen Gulf. Still, our data show that the relative contribution of fossil and $\mathrm{C}_{3}$ terrestrial plants increased with water column depth, certainly due to the degradation of the algal components within the water column.

\subsection{Transport and fate of particulate matter}

Patterns of lipid categories throughout the water column and in the sediment were studied at the slope sites 235 and 345. Figure 9 (site 235) shows dramatically different patterns between the deep chlorophyll maximum $(\sim 70 \mathrm{~m})$ in the upper Pacific halocline, the lower Atlantic halocline $(\sim 200 \mathrm{~m})$ and the sediment. Flagellates dominated at the depth of maximum chlorophyll, and diatom biomarkers became relatively more important at $200 \mathrm{~m}$ and in sediment. This suggests postbloom conditions within the water column with only larger phytoplankton exported to deeper depths and sediments. This is consistent with the well-known fact that picoplankton are efficiently recycled within the food web and only large phytoplankton are exported (Michaels and Silver, 1988). Moreover, the enriched carbon isotope values obtained for the sedimentary diatom markers imply high rates of primary production. These features of site 235 were also observed at site 345 .

Selective loss with water column depth of the more labile organic compounds with respect to organic carbon is related to degradation processes during the transport of particles into deep water (Tolosa et al., 2004b; Wakeham et al., 1997). While maximum values of PUFA and phytol were observed around the DCM, the maxima for the bacterial signatures shifted down to 130-200 m (Fig. 10). As expected, the ratio PUFA/(SCFA + SCMUFA), an index of freshness of organic matter, exhibited the highest values (fresher material) at the deep chlorophyll maximum $(\sim 70 \mathrm{~m})$, with the exception of sample 640-70, which seemed to be affected by the Mackenzie River plume. The minimum values were noticed in the samples collected within the Pacific halocline water mass at $130 \mathrm{~m}$ depth, coinciding with the highest percentage of bacterial markers. These observations might be explained by the remineralization of organic matter that could produce the nutrient maximum and oxygen minimum in this layer (Cota et al., 1996).

The distribution of TOC-normalized concentration of biomarkers in the surface sediment did not differ from that based on absolute concentrations of lipids. As shown in Fig. 11, the concentrations of fresh/labile algal biomarkers (PUFA, phytol) sharply decreased by $\sim 80 \%$ from the inner to the outer shelf including the Amundsen Gulf site, and to the slope. Concentrations of bacterial (BrFA) and zooplankton (LCMUFA) biomarkers were markedly higher in the inner shelf than at the other sites. Allochthonous biomarkers, e.g. LCFA from $\mathrm{C}_{3}$ plant and fossil biomarkers, were highest in the inner shelf, lowest in the outer shelf and Amundsen Gulf and increased again in the slope. As expected, the highest index of freshness (PUFA/(SCFA + SCMUFA) was measured in the shallow areas of the inner shelf, and decreased with water column depth (with the exception of site 345). This decrease of lipid concentrations with water column depth suggests that labile algal material is subject to degradation and recycling by bacteria throughout the water 

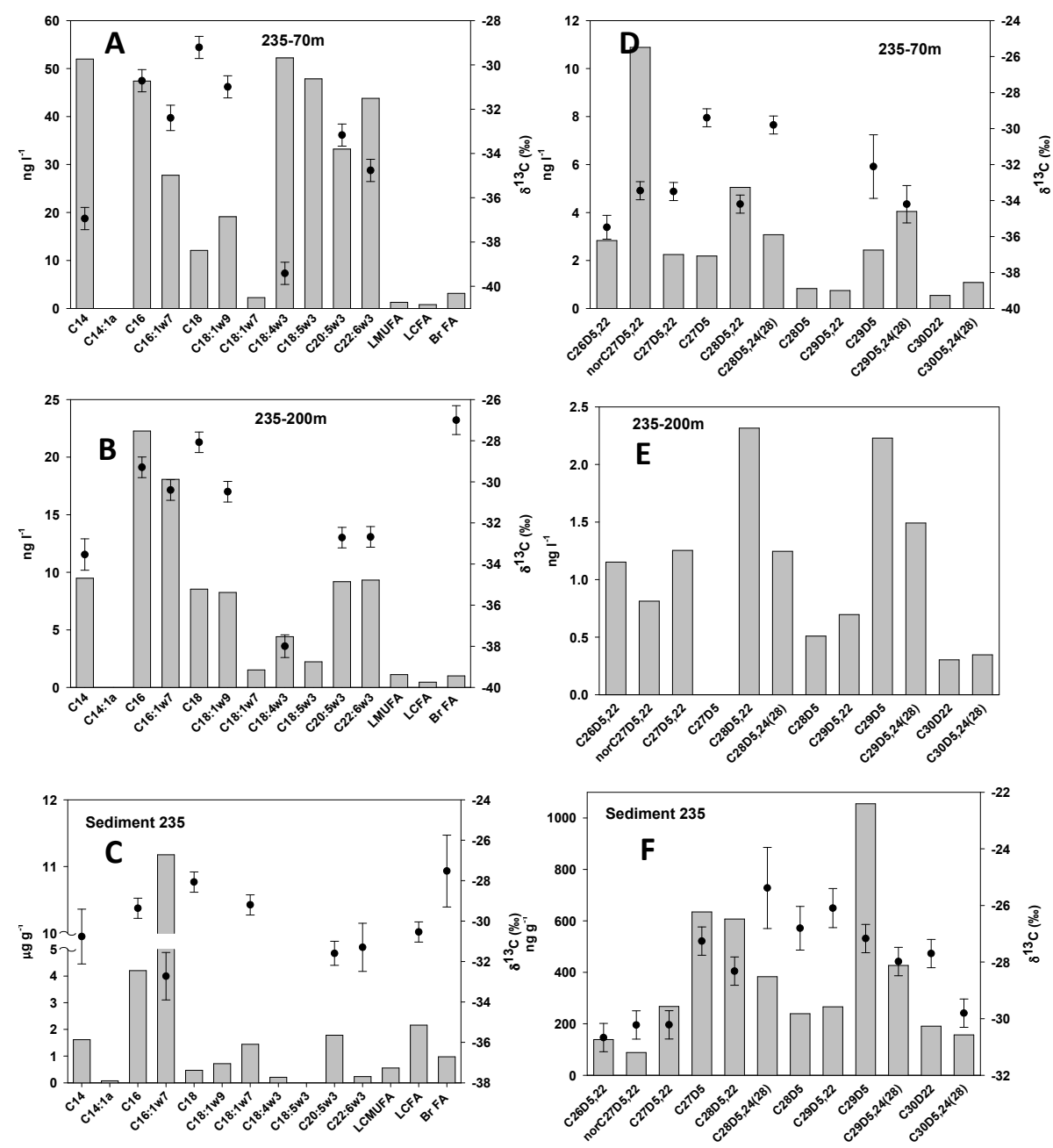

Fig. 9. Concentrations (bars) and carbon isotopic ratios (mean $\pm \mathrm{SD}, n=3$ ) of selected fatty acids (A-C) and sterols (D-F) in a depth profile from site 235 .

column before being stored in the sediment floor. However, the presence of labile compounds in the sediments lying hundreds of meters below the ocean surface is probably due to a rapid sinking of ungrazed phytoplankton from blooms of diatoms or ice algae mats released when ice melts. If these compounds were deposited and resuspended en route to the deep basins, they would be degraded for periods of time long enough to be lost (Yunker et al., 2005). As FAs degrade faster than $n$-alkanes, the ratios of long-chain $n$-alkanoic acids to $n$ alkanes indicated a higher preservation of the terrestrial OM in the inner shelf compared to the outer shelf where more degraded or refractory terrestrial material was found. These terrigenous biomarkers preserved in the sediments might derive from fluvial or eroded shoreline sedimentary organic matter that has been carried out offshore by advective particle transport, e.g. nepheloid layers (Forest et al., 2007; Honjo et al., 2010). Moreover, the inverse distribution between retene and $\alpha$-amyrin (Fig. 12) might indicate a different transport of these two terrestrial compounds, presumably arising from their precursors and association with particles. $\alpha$-amyrin, a specific biomarker for angiosperms (Hernes and Hedges, 2004), is linked to bound fractions and associated with lowdensity, higher plant debris. It might therefore be widely dispersed by the Mackenzie River and be preserved out to the shelf edge and slope. In contrast, retene (which is a dominant component of coals) is preferentially linked to diagenetic PAHs associated with lithic particles that settled out in nearshore sediments (Yunker et al., 1995).

\subsection{Estimation of allochthonous organic carbon content in sediments}

The organic content of deep surface sediments is anomalously high in the Beaufort Sea (Magen et al., 2010) certainly due to the addition of terrestrial plants and fossil material through river transport to the shelves and basins and to the tight pelagic-benthic coupling. The percentage of allochthonous carbon (\% C-terr) in marine sediments may be 


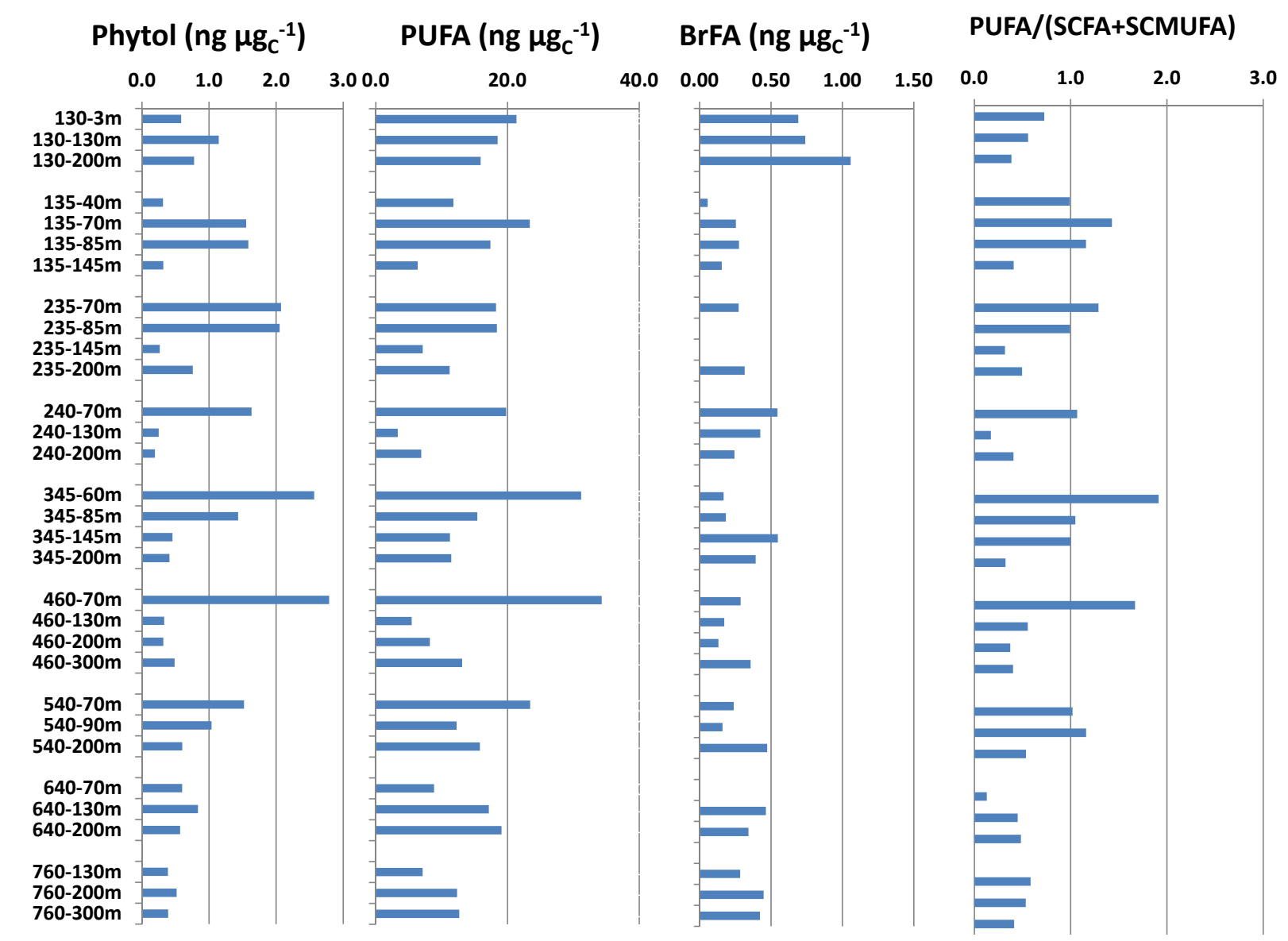

Fig. 10. Concentrations normalized to TOC of selected lipid biomarkers and diagnostic ratios in the SPM of offshore open waters ( $>200 \mathrm{~m}$ depth) from the Mackenzie slope.

obtained from the ratio of an allochthonous biomarker (normalized to TOC) between marine sediments and the river mouth water (Bouloubassi et al., 1997; Saliot et al., 2002). This approach assumes that the allochthonous biomarkers are conservative and that the only factor affecting the ratio between allochthonous biomarkers and terrestrial organic carbon in sediments is dilution with the marine organic carbon. Table 13 shows the range in estimated \% C-terr that resulted from our samples using this approach. Using the wax $n$-alkanes, 30 to $37 \%$ of the organic carbon is of terrestrial origin in the inner shelf sediments (sites 690 and 390), and 20 to $27 \%$ in the sediments from the outer shelf, Amundsen Gulf and slope. These values are much lower than those estimated in 1987 using the same approach where inner shelf and slope sediments contained $99 \%$ and $62 \%$ of terrestrial carbon, respectively (Belicka et al., 2004). Even when using other $\mathrm{C}_{3}$ terrestrial biomarkers (Table 13), mean values in the inner shelf were 30-35\% (sitosterol excluded at 390) and between 15 and $25 \%$ at the other sites. The variability between the different biomarkers is likely due to the different extent of the degradation process of terrigenous biomarkers, $n$-alkanes being more stable than their oxygenated precursors
( $n$-alkanols and $n$-alkanoic acids) and $n$-alkanoic acids more stable than $n$-alcohols (Cranwell, 1981). Sitosterol exhibited similar percentages as the other terrestrial biomarkers except for site 390, where phytoplankton were the major source of this compound. Although fossil hydrocarbons might be generated also within the sediments, the percentage of fossil carbon preserved in the sediments was similar to that resulting from the $\mathrm{C}_{3}$ plant biomarkers. Regardless of the compound series, the percentage of organic carbon preserved in the sediments decreased from the nearshore to offshore sites.

Our estimates of the allochthonous carbon content are low compared to other studies conducted 5-20 yr earlier and using the same (Belicka et al., 2004) or different approaches. The latter ones reported values mostly $>50 \%$ (Magen et al., 2010; Macdonald et al., 1998; Goñi et al., 2000), some of them distinguishing between the fossil (40-70\%) and the vascular C3 plant $(<30 \%)$ contribution (Drenzek et al., 2007; Goñi et al., 2005). Compared to the study of Belicka et al. (2004), the carbon content of our sediments could have been overestimated by the relatively higher contribution of labile components due to the sampling of only the topmost layer (few mm). However, when taking into account 


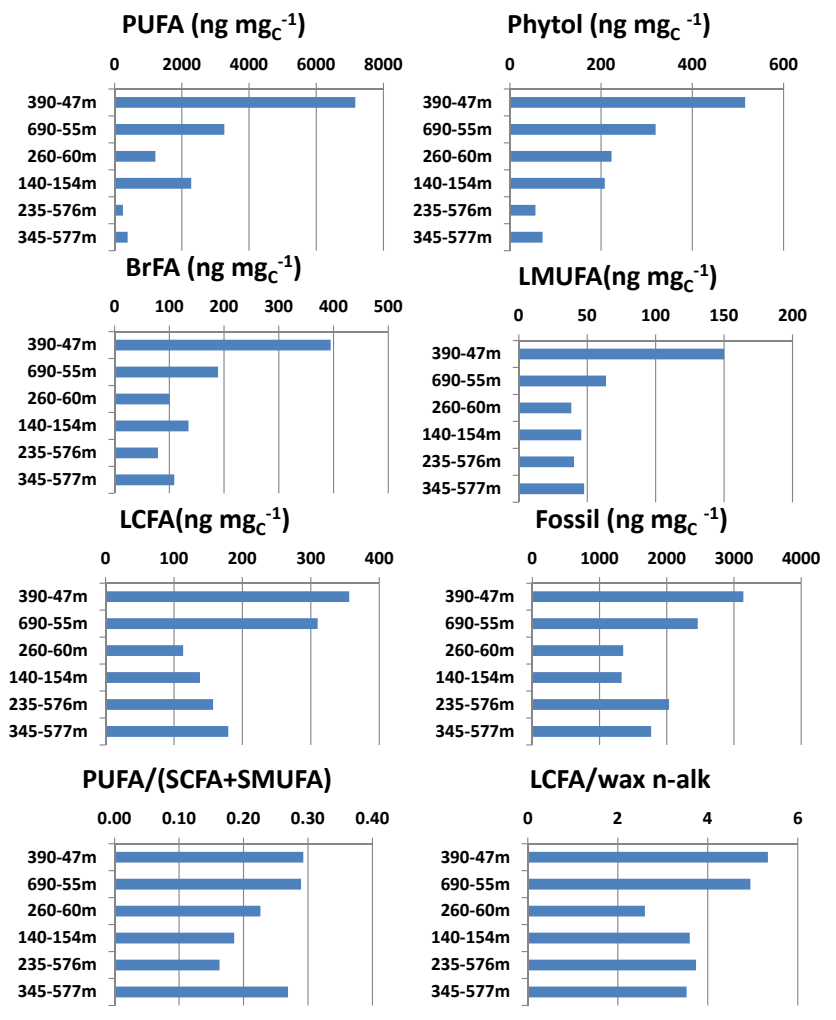

Fig. 11. TOC-normalized concentration of selected biomarkers and selected diagnostic ratios in surface sediments from the shelf, Amundsen Gulf and slope.

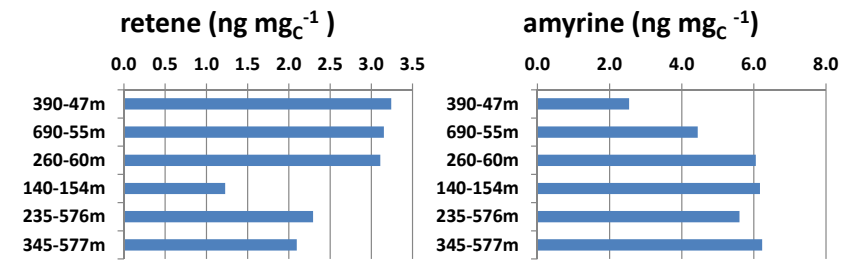

Fig. 12. TOC-normalized concentration of retene and $\alpha$-amyrin in surface sediments from the shelf, Amundsen Gulf and slope.

a maximum overestimation of $20 \%$ in the TOC from our upper sediments, this would result in an increase of the terrestrial contribution of only 4 to $12 \%$. Therefore more plausible reasons for the decrease of the terrestrial contribution in recent sediments are that the annual discharge of the Mackenzie River has actually decreased in the last years/decades (Durantou et al., 2012) and that primary production over the Canadian Beaufort Shelf has increased during the last decade. Recent works suggest that the Arctic Ocean carbon sink has tripled from 1972 to 2002 mainly because of the ice cover removal that maintained surface waters undersaturated with respect to $\mathrm{CO}_{2}$ (Bates et al., 2006), and that annual primary production has increased by $25 \%$ from 1998 to 2007 (Arrigo et al., 2008; Pabi et al., 2008) and quadrupled from 2004 to 2008 in the inner Mackenzie Shelf (Tremblay et al.,
Table 13. Percentage of terrigenous organic carbon with respect to the total organic content of the sediment sample, obtained from the ratio of different terrestrial biomarker concentrations (normalized to total organic carbon) at a given site versus the biomarker concentrations representative of the river.

\begin{tabular}{lrrrrrr}
\hline & 390 & 690 & 260 & 140 & 235 & 345 \\
& $47 \mathrm{~m}$ & $55 \mathrm{~m}$ & $60 \mathrm{~m}$ & $154 \mathrm{~m}$ & $576 \mathrm{~m}$ & $577 \mathrm{~m}$ \\
\hline Wax $n$-alkanes & 31 & 37 & 23 & 20 & 22 & 27 \\
LCOH & 20 & 21 & 13 & 13 & 15 & 19 \\
LCFA & 47 & 41 & 15 & 18 & 21 & 24 \\
Sitosterol & 93 & 27 & 16 & 18 & 14 & 27 \\
Fossil $^{*}$ & 39 & 30 & 17 & 16 & 25 & 22 \\
\hline
\end{tabular}

*Fossil compounds include the unresolved complex mixture (UCM), petrol $n$-alkanes (wax $n$-alkanes were subtracted from total $n$-alkanes), pristane and phytane.

2011). This increase in annual production has been attributed to the longer phytoplankton growing season due to the increase in the number of ice-free days and to the strength and persistence of winds favouring upwelling. More data will be needed to monitor these trends and evaluate the associated changes.

\section{Summary and conclusions}

The measurement of lipid biomarkers and their compoundspecific isotope analysis allowed us to characterize the spatial variation of OM over the Mackenzie Shelf and the slope to better constrain the sources of terrestrial and marine organic matter. Our data highlight that fresh and labile organic matter from diatom blooms sinks to the bottom of the continental shelf and slope, whereas terrestrial material is likely transported to the slope by advective processes. Although sitosterol is generally considered to be of terrestrial origin, the carbon isotope ratios we obtained for this compound at site 390 indicated a high autochthonous production. Since $\delta^{13} \mathrm{C}$ values obtained for marine phytoplanktonic biomarkers synthesized at this high latitude area with relatively high concentrations of $\mathrm{CO}_{2}$ might be similarly depleted as the $\delta^{13} \mathrm{C}$ values of $\mathrm{C}_{3}$ terrestrial biomarkers, it is problematic to discern the sources of sitosterol in the marine SPM by using their $\delta^{13} \mathrm{C}$.

Although the Mackenzie River is the primary source of $\mathrm{C}_{3}$ terrigenous debris and fossil material to the Mackenzie Shelf sediments, refractory algae-derived material was the major lipidic component in the nearshore sediments. However, their relative contributions decreased with water column depth, which lead to an increase in the contributions of fossil and $\mathrm{C}_{3}$ plant-derived material.

Our evaluations on the terrigenous POC fraction preserved in the surface sediments of the Beaufort Sea compared to studies prior to the recent decline in Arctic summertime ice indicate a decrease during the last decade implying a recent shift between autochthonous and allochthonous source input 
over the sediments. Interestingly, these results are supported by the enhancement of the primary production in the Arctic Ocean in recent years. Our data provide an important baseline for future studies.

Acknowledgements. The IAEA is grateful for the support provided to the Environment Laboratories by the Government of the Principality of Monaco. This study was conducted as part of the Malina Scientific Program funded by ANR (Agence nationale de la recherche), INSU-CNRS (Institut national des sciences de l'univers - Centre national de la recherche scientifique), CNES (Centre national d'études spatiales) and ESA (European Space Agency). The authors would like to thank M. Babin, chief scientist of the cruise and coordinator of the Malina program, A. Forest, H. Link and G. Chaillou and three anonymous reviewers for their constructive comments.

Edited by: B. A. Bergamaschi

\section{References}

Amiel, D. and Cochran, J. K.: Terrestrial and marine POC fluxes derived from 234Th distributions and $\delta^{13} \mathrm{C}$ measurements on the Mackenzie Shelf, J. Geophys. Res., 113, C03S06, doi:10.1029/2007jc004260, 2008.

Arrigo, K. R., van Dijken, G., and Pabi, S.: Impact of a shrinking Arctic ice cover on marine primary production, Geophys. Res. Lett., 35, L19603, doi:10.1029/2008g1035028, 2008.

Baas, M., Pancost, R., van Geel, B., and Sinninghe Damste, J. S.: A comparative study of lipids in Sphagnum species, Org. Geochem., 31, 535-541, 2000.

Baker, E. W. and Louda, J. W.: Thermal aspects in chlorophyll geochemistry, Advances in Organic Geochemistry, edited by: Bjorøy, M., 1981, Wiley, Chichester, 401-421, 1983.

Balzano, S., Gourvil, P., Siano, R., Chanoine, M., Marie, D., Lessard, S., Sarno, D., and Vaulot, D.: Diversity of cultured photosynthetic flagellates in the northeast Pacific and Arctic Oceans in summer, Biogeosciences, 9, 4553-4571, doi:10.5194/bg-94553-2012, 2012.

Barber, D. G. and Hanesiak, J. M.: Meteorological forcing of sea ice concentrations in the southern Beaufort Sea over the period 1979 to 2000, J. Geophys. Res., 109, C06014, doi:10.1029/2003jc002027, 2004.

Barrett, S. M., Volkman, J. K., Dunstan, G. A., and LeRoi, J.-M.: Sterols of 14 species of marine diatoms (bacillariophyta), J. Phycol., 31, 360-369, 1995.

Bates, N. R., Moran, S. B., Hansell, D. A., and Mathis, J. T.: An increasing $\mathrm{CO}_{2}$ sink in the Arctic Ocean due to sea-ice loss, Geophys. Res. Lett., 33, L23609, doi:10.1029/2006g1027028, 2006.

Belicka, L. L., Macdonald, R. W., Yunker, M. B., and Harvey, H. R.: The role of depositional regime on carbon transport and preservation in Arctic Ocean sediments, Mar. Chem., 86, 65-88, 2004.

Belt, S. T., Masse, G., Rowland, S. J., Poulin, M., Michel, C., and LeBlanc, B.: A novel chemical fossil of palaeo sea ice: IP25, Org. Geochem., 38, 16-27, 2007.

Belt, S. T., Massé, G., Vare, L. L., Rowland, S. J., Poulin, M., Sicre, M.-A., Sampei, M., and Fortier, L.: Distinctive 13C isotopic sig- nature distinguishes a novel sea ice biomarker in Arctic sediments and sediment traps, Mar. Chem., 112, 158-167, 2008.

Benthien, A., Zondervan, I., Engel, A., Hefter, J., Terbruggen, A., and Riebesell, U.: Carbon isotopic fractionation during a mesocosm bloom experiment dominated by Emiliania huxleyi: Effects of $\mathrm{CO}_{2}$ concentration and primary production, Geochim. Cosmochim. Ac., 71, 1528-1541, 2007.

Bi, X., Sheng, G., Liu, X., Li, C., and Fu, J.: Molecular and carbon and hydrogen isotopic composition of n-alkanes in plant leaf waxes, Org. Geochem., 36, 1405-1417, 2005.

Bouloubassi, I., Lipiatou, E., Saliot, A., Tolosa, I., Bayona, J. M., and Albaiges, J.: Carbon sources and cycle in the western Mediterranean - the use of molecular markers to determine the origin of organic matter, Deep Sea Res. II, 44, 781-799, 1997.

Boutton, T. W.: Stable carbon isotope ratios of natural materials: II. Atmospheric, terrestrial, marine, and freshwater environments, in: Carbon isotope techniques, edited by: Colleman, D. C. and Fry, B., Academic Press, Inc, 173-185, 1991.

Bray, E. E. and Evans, E. D.: Distribution of n-paraffins as a clue to recognition of source beds, Geochim. Cosmochim. Ac., 22, 2-15, 1961.

Brugel, S., Nozais, C., Poulin, M., Tremblay, J. E., Miller, L. A., Simpson, K. G., Gratton, Y., and Demers, S.: Phytoplankton biomass and production in the southeastern Beaufort Sea in autumn 2002 and 2003, Mar. Ecol.-Prog. Ser., 377, 63-77, doi:10.3354/meps07808, 2009.

Carmack, E., Macdonald, C., Robie, W., and Jasper, S.: Phytoplankton productivity on the Canadian Shelf of the Beaufort Sea, Mar. Ecol.-Prog. Ser., 277, 37-50, doi:10.3354/meps277037, 2004.

Carmack, E., Barber, D., Christensen, J., Macdonald, R., Rudels, B., and Sakshaug, E.: Climate variability and physical forcing of the food webs and the carbon budget on panarctic shelves, Prog. Oceanogr., 71, 145-181, 2006.

Carrie, J., Sanei, H., Goodarzi, F., Stern, G., and Wang, F.: Characterization of organic matter in surface sediments of the Mackenzie River Basin, Canada, Int. J. Coal Geol., 77, 416-423, 2009.

Chikaraishi, Y. and Naraoka, H.: Compound-specific $\delta \mathrm{D}-\delta{ }^{13} \mathrm{C}$ analyses of $n$-alkanes extracted from terrestrial and aquatic plants, Phytochemistry, 63, 361-371, 2003.

Chikaraishi, Y. and Naraoka, H.: $\delta^{13} \mathrm{C}$ and $\delta \mathrm{D}$ identification of sources of lipid biomarkers in sediments of Lake Haruna (Japan), Geochim. Cosmochim. Ac., 69, 3285-3297, 2005.

Chikaraishi, Y. and Naraoka, H.: $\delta^{13} \mathrm{C}$ and $\delta \mathrm{D}$ relationships among three n-alkyl compound classes (n-alkanoic acid, n-alkane and n-alkanol) of terrestrial higher plants, Org. Geochem., 38, 198215, 2007.

Cloern, J. E., Canuel, E. A., and Harris, D.: Stable carbon and nitrogen isotope composition of aquatic and terrestrial plants of the San Francisco Bay estuarine system, Limnol. Oceanogr., 47, 713-729, 2002.

Codispoti, L. A., Flagg, C., Kelly, V., and Swift, J. H.: Hydrographic conditions during the 2002 SBI process experiments, Deep Sea Res. II, 52, 3199-3226, 2005.

Collister, J. W., Rieley, G., Stern, B., Eglinton, G., and Fry, B.: Compound-specific $\delta^{13} \mathrm{C}$ analyses of leaf lipids from plants with differing carbon dioxide metabolisms, Org. Geochem., 21, 619627, 1994.

Cota, G. F., Pomeroy, L. R., Harrison, W. G., Jones, E. P., Peters, F., Sheldon Jr., W. M., and Weingartner, T. R.: Nutrients, pri- 
mary production and microbial heterotrophy in the southeastern Chukchi Sea: Arctic summer nutrient depletion and heterotrophy, Mar. Ecol.-Prog. Ser., 135, 247-258, doi;10.3354/meps135247, 1996.

Cranwell, P. A.: Diagenesis of free and bound lipids in terrestrial detritus deposited in a lacustrine sediment, Org. Geochem., 3, 79-89, 1981.

Dalsgaard, J., John, M. S., Kattner, G., Müller-Navarra, D., and Hagen, W.: Fatty acid trophic markers in the pelagic marine environment, Adv. Mar. Biol., 46, 225-340, 2003.

Doxaran, D., Ehn, J., Bélanger, S., Matsuoka, A., Hooker, S., and Babin, M.: Optical characterisation of suspended particles in the Mackenzie River plume (Canadian Arctic Ocean) and implications for ocean colour remote sensing, Biogeosciences, 9, 32133229, doi:10.5194/bg-9-3213-2012, 2012.

Drenzek, N. J., Montlucon, D. B., Yunker, M. B., Macdonald, R. W., and Eglinton, T. I.: Constraints on the origin of sedimentary organic carbon in the Beaufort Sea from coupled molecular ${ }^{13} \mathrm{C}$ and ${ }^{14} \mathrm{C}$ measurements, Mar. Chem., 103, 146-162, 2007.

Dunstan, G. A., Volkman, J. K., Jeffrey, S. W., and Barrett, S. M.: Biochemical composition of microalgae from the green algal classes Chlorophyceae and Prasinophyceae, 2. Lipid classes and fatty acids, J. Exp. Mar. Biol. Ecol., 161, 115-134, 1992.

Durantou, L., Rochon, A., Ledu, D., Massé, G., Schmidt, S., and Babin, M.: Quantitative reconstruction of sea-surface conditions over the last $150 \mathrm{yr}$ in the Beaufort Sea based on dinoflagellate cyst assemblages: the role of large-scale atmospheric circulation patterns, Biogeosciences, 9, 5391-5406, doi:10.5194/bg-9-53912012, 2012.

Dyke, L. D. and Brooks, G. R.: The physical environment of the Mackenzie Valley, Northwest Territories: a base line for the assessment of environmental change, Bull. Geol. Surv. Can., 547, 208 pp., 2000.

Fahl, K. and Stein, R.: Modern organic carbon deposition in the Laptev Sea and the adjacent continental slope: surface water productivity vs. terrigenous input, Org. Geochem., 26, 379-390, 1997.

Forest, A., Sampei, M., Hattori, H., Makabe, R., Sasaki, H., Fukuchi, M., Wassmann, P., and Fortier, L.: Particulate organic carbon fluxes on the slope of the Mackenzie Shelf (Beaufort Sea): Physical and biological forcing of shelf-basin exchanges, J. Marine Syst., 68, 39-54, 2007.

Forest, A., Sampei, M., Makabe, R., Sasaki, H., Barber, D. G., Gratton, Y., Wassmann, P., and Fortier, L.: The annual cycle of particulate organic carbon export in Franklin Bay (Canadian Arctic): Environmental control and food web implications, J. Geophys. Res., 113, C03S05, doi:10.1029/2007jc004262, 2008.

Forest, A., Babin, M., Stemmann, L., Picheral, M., Sampei, M., Fortier, L., Gratton, Y., Bélanger, S., Devred, E., Sahlin, J., Doxaran, D., Joux, F., Ortega-Retuerta, E., Jeffrey, W. H., Martín, J., Gasser, B. and Miquel, J. C.: Ecosystem function and particle flux dynamics across the Mackenzie Shelf (Beaufort Sea, Arctic Ocean): an integrative analysis of spatial variability and biophysical forcings, Biogeosciences Discuss., 9, 10883-10960, doi:10.5194/bgd-9-10883-2012, 2012.

Goad, L. and Withers, N.: Identification of 27-nor-(24R)-24methylcholesta-5, 22-dien-3 $\beta$-ol and brassicasterol as the major sterols of the marine dinoflagellate Gymnodinium simplex, Lipids, 17, 853-858, doi:10.1007/bf02534578, 1982.
Goericke, R. and Fry, B.: Variations of marine plankton $\delta^{13} \mathrm{C}$ with latitude, temperature, and dissolved $\mathrm{CO}_{2}$ in the world ocean, Global Biogeochem. Cy., 8, 85-90, doi:10.1029/93gb03272, 1994.

Goñi, M. A., Yunker, M. B., Macdonald, R. W., and Eglinton, T. I.: Distribution and sources of organic biomarkers in arctic sediments from the Mackenzie River and Beaufort Shelf, Mar. Chem., 71, 23-51, 2000.

Goñi, M. A., Yunker, M. B., Macdonald, R. W., and Eglinton, T. I.: The supply and preservation of ancient and modern components of organic carbon in the Canadian Beaufort Shelf of the Arctic Ocean, Mar. Chem., 93, 53-73, 2005.

Gradinger, R.: Sea-ice algae: Major contributors to primary production and algal biomass in the Chukchi and Beaufort Seas during May/June 2002, Deep Sea Res.II, 56, 1201-1212, 2009.

Grimalt, J. O., Torras, E., and Albaigés, J.: Bacterial reworking of sedimentary lipids during sample storage, Org. Geochem., 13, 741-746, doi:10.1016/0146-6380(88)90096-4, 1988.

Grimalt, J. O. and Albaigés, J.: Characterization of the depositional environments of the Ebro Delta (western Mediterranean) by the study of sedimentary lipid markers, Mar. Geol., 95, 207-224, 1990.

Han, J. and Calvin, M.: Hydrocarbon Distribution of Algae and Bacteria, and Microbiological Activity in Sediments, P. Natl. Acad. Sci. USA, 64, 436-443, doi:10.1073/pnas.64.2.436, 1969.

Hargrave, B. T., Walsh, I. D. and Murray, D. W.: Seasonal and spatial patterns in mass and organic matter sedimentation in the North Water, Deep Sea Res. II, 49, 5227-5244, 2002.

Harvey, H. R., Eglinton, G., O'Hara, S. C. M., and Corner, E. D. S.: Biotransformation and assimilation of dietary lipids by Calanus feeding on a dinoflagellate, Geochim. Cosmochim. Ac., 51, 3031-3040, 1987.

Hayes, J. M.: Fractionation of the isotopes of carbon and hydrogen in biosynthetic processes, in: Stable Isotope Geochemistry, Reviews in Mineralogy and Geochemistry, edited by: Valley, J. W. and Cole, D. R., 43, Mineralogical Society of America, 225-278, 2001.

Hernes, P. J. and Hedges, J. I.: Tannin signatures of barks, needles, leaves, cones, and wood at the molecular level, Geochim. Cosmochim. Ac., 68, 1293-1307, 2004.

Honjo, S., Krishfield, R. A., Eglinton, T. I., Manganini, S. J., Kemp, J. N., Doherty, K., Hwang, J., McKee, T. K., and Takizawa, T.: Biological pump processes in the cryopelagic and hemipelagic Arctic Ocean: Canada Basin and Chukchi Rise, Prog. Oceanogr., 85, 137-170, 2010.

Iken, K., Bluhm, B. A., and Gradinger, R.: Food web structure in the high Arctic Canada Basin: evidence from $\delta^{13} \mathrm{C}$ and $\delta^{15} \mathrm{~N}$ analysis, Polar Biol., 28, 238-249, doi:10.1007/s00300-004-0669-2, 2005.

Johannessen, O. M., Bengtsson, L., Miles, M. W., Kuzmina, S. I., Semenov, V. A., Alekseev, G. V., Nagurnyi, A. P., Zakharov, V. F., Bobylev, L. P., Pettersson, L. H., Hasselmann, K., and Cattle, H. P.: Arctic climate change: observed and modelled temperature and sea-ice variability, Tellus A, 56, 328-341, 2004.

Jones, D. M., Carter, J. F., Eglinton, G., Jumeau, E. J., and Fenwick, C. S.: Determination of $\mathrm{d}^{13} \mathrm{C}$ values of sedimentary straight chain and cyclic alcohols by gas chromatography/isotope ratio mass spectrometry, Biol. Mass Spectrom., 20, 641-646, 1991. 
Lansard, B., Mucci, A., Miller, L. A., Macdonald, R. W., and Gratton, Y.: Seasonal variability of water mass distribution in the southeastern Beaufort Sea determined by total alkalinity and $\delta^{18} \mathrm{O}$, J. Geophys. Res., 117, C03003, doi:10.1029/2011JC007299, 2012.

Lee, R. F., Hagen, W., and Kattner, G.: Lipid storage in marine zooplankton. Mar. Ecol.-Prog. Ser., 307, 273-306, 2006.

Lewis, E. and Wallace, D. W. R.: Program Developed for $\mathrm{CO}_{2}$ System Calculations. ORNL/CDIAC-105, Carbon Dioxide Information Analysis Center, Oak Ridge National Laboratory, US Department of Energy, Oak Ridge, Tennessee, 1998.

Lovejoy, C., Vincent, W. F., Bonilla, S., Roy, S., Martineau, M.-J., Terrado, R., Potvin, M., Massana, R., and Pedrós-Alió, C.: Distribution, phylogeny, and growth of cold-adapted picoprasinophytes in Artic Seas, J. Phycol., 43, 78-89, doi:10.1111/j.15298817.2006.00310.x, 2007.

Macdonald, R. W., Solomon, S. M., Cranston, R. E., Welch, H. E., Yunker, M. B., and Gobeil, C.: A sediment and organic carbon budget for the Canadian Beaufort Shelf, Mar. Geol., 144, 255273, 1998 .

Magen, C., Chaillou, G., Crowe, S. A., Mucci, A., Sundby, B., Gao, A., Makabe, R., and Sasaki, H.: Origin and fate of particulate organic matter in the southern Beaufort Sea - Amundsen Gulf region, Canadian Arctic, Estuarine, Coast. Shelf Sci., 86, 31-41, 2010.

Martín, J., Sanchez-Cabeza, J. A., Eriksson, M., Levy, I., and Miquel, J. C.: Recent accumulation of trace metals in sediments at the DYFAMED site (Northwestern Mediterranean Sea), Mar. Pollut. Bull., 59, 146-153, 2009.

Matsuoka, A., Bricaud, A., Benner, R., Para, J., Sempéré, R., Prieur, L., Bélanger, S., and Babin, M.: Tracing the transport of colored dissolved organic matter in water masses of the Southern Beaufort Sea: relationship with hydrographic characteristics, Biogeosciences, 9, 925-940, doi:10.5194/bg-9-925-2012, 2012.

Michaels, A. F. and Silver, M. W.: Primary production, sinking fluxes and the microbial food web, Deep Sea Research A, 35, 473-490, 1988.

Miquel, J. C., Fowler, S. W., La Rosa, J., and Buat-Menard, P.: Dynamics of the downward flux of particles and carbon in the open northwestern Mediterranean Sea, Deep Sea Res. I, 41, 243-261, 1994.

Morata, N., Renaud, P. E., Brugel, S., Hobson, K. A., and Johnson, B. J.: Spatial and seasonal variations in the pelagicbenthic coupling of the southeastern Beaufort Sea revealed by sedimentary biomarkers, Mar. Ecol.-Prog. Ser., 371, 47-63, doi:10.3354/meps07677, 2008.

Mucci, A., Lansard, B. Miller, L. A. and Papakyriakou T.: $\mathrm{CO}_{2}$ fluxes across the air-sea interface in the southeastern Beaufort Sea: Ice-free period, J. Geophys. Res., 115, C04003, doi:10.1029/2009JC005330, 2010.

Mundy, C. J., Gosselin, M., Ehn, J., Gratton, Y., Rossnagel, A., Barber, D. G., Martin, J., Tremblay, J.-É., Palmer, M., Arrigo, K. R., Darnis, G., Fortier, L., Else, B., and Papakyriakou, T.: Contribution of under-ice primary production to an ice-edge upwelling phytoplankton bloom in the Canadian Beaufort Sea, Geophys. Res. Lett., 36, L17601, doi:10.1029/2009g1038837, 2009.

Murata, A.: Increased surface seawater $p \mathrm{CO}_{2}$ in the eastern Bering Sea shelf: An effect of blooms of coccolithophorid Emiliania huxleyi?, Global Biogeochem. Cy., 20, GB4006, doi:10.1029/2005gb002615, 2006.

Naidu, A. S., Cooper, L. W., Finney, B. P., Macdonald, R. W., Alexander, C., and Semiletov, I. P.: Organic carbon isotope ratios $\delta^{13} \mathrm{C}$ ) of Arctic Amerasian Continental shelf sediments, Int J. Earth Sci., 89, 522-532, doi:10.1007/s005310000121, 2000.

Nishimura, M.: The geochemical significance in early sedimentation of geolipids obtained by saponification of lacustrine sediments, Geochim. Cosmochim. Ac., 41, 1817-1823, 1977.

O’Brien, M. C., Macdonald, R. W., Melling, H., and Iseki, K.: Particle fluxes and geochemistry on the Canadian Beaufort Shelf: Implications for sediment transport and deposition, Cont. Shelf Res., 26, 41-81, 2006.

Ortega-Retuerta, E., Jeffrey, W. H., Babin, M., Bélanger, S., Benner, R., Marie, D., Matsuoka, A., Raimbault, P., and Joux, F.: Carbon fluxes in the Canadian Arctic: patterns and drivers of bacterial abundance, production and respiration on the Beaufort Sea margin, Biogeosciences, 9, 3679-3692, doi:10.5194/bg-9-36792012, 2012.

Pabi, S., van Dijken, G. L., and Arrigo, K. R.: Primary production in the Arctic Ocean, 1998-2006, J. Geophys. Res., 113, C08005, doi:10.1029/2007jc004578, 2008.

Pfirman, S. L., Eicken, H., Bauch, D., and Weeks, W. F.: The potential transport of pollutants by Arctic sea ice, Sci. Total Environ., 159, 129-146, 1995.

Rachold, V., Grigoriev, M. N., Are, F. E., Solomon, S., Reimnitz, E., Kassens, H., and Antonow, M.: Coastal erosion vs riverine sediment discharge in the Arctic Shelf seas, Int. J. Earth Sci., 89, 450-460, doi:10.1007/s005310000113, 2000.

Rachold, V., Eicken, H., Gordeev, V. V., Grigoriev, M. N., Hubberten, H.-W., Lisitzin, A. P., Shevchenko, V. P., and Schirrmeister, L.: Modern terrigenous organic carbon input to the Arctic Ocean, in: The Organic Carbon Cycles in the Arctic Ocean, edited by: Stein, R. and Macdonald, R. W., Springer, Berlin, Heidelberg, 35-55, 2004.

Raimbault, P., Garcia, N., and Cerutti, F.: Distribution of inorganic and organic nutrients in the South Pacific Ocean - evidence for long-term accumulation of organic matter in nitrogendepleted waters, Biogeosciences, 5, 281-298, doi:10.5194/bg-5281-2008, 2008.

Ras, J., Claustre, H., and Uitz, J.: Spatial variability of phytoplankton pigment distributions in the Subtropical South Pacific Ocean: comparison between in situ and predicted data, Biogeosciences, 5, 353-369, doi:10.5194/bg-5-353-2008, 2008.

Rielley, G., Collier, R. J., Jones, D. M., and Eglinton, G.: The biogeochemistry of Ellesmere Lake, U.K. I: source correlation of leaf wax inputs to the sedimentary lipid record, Org. Geochem., 17, 901-912, 1991.

Robinson, N., Eglinton, G., Brassell, S. C. and Cranwell, P. A.: Dinoflagellate origin for sedimentary 4amethylsteroids and $5 \alpha(\mathrm{H})$ stanols, Nature, 308, 419-422, 1984a.

Robinson, N., Cranwell, P. A., Finlay, B. J., and Eglinton, G.: Lipids of aquatic organisms as potential contributors to lacustrine sediments, Org. Geochem., 6, 143-152, 1984b.

Ruttenberg, K. C. and Goñi, M. A.: Phosphorus distribution, $\mathrm{C}: \mathrm{N}: \mathrm{P}$ ratios, and $\delta^{13} \mathrm{C}_{\mathrm{oc}}$ in arctic, temperate, and tropical coastal sediments: tools for characterizing bulk sedimentary organic matter, Mar. Geol., 139, 123-145, 1997.

Saliot, A., Parrish, C. C., Sadouni, N., Bouloubassi, I., Fillaux, J., and Cauwet, G.: Transport and fate of Danube Delta terrestrial 
organic matter in the Northwest Black Sea mixing zone, Mar. Chem., 79, 243-259, 2002.

Schouten, S., Klein Breteler, W. C. M., Blokker, P., Schogt, N. C., Rijpstra, W. I., Grice, K., Baas, M. and Sinninghe Damste, J. S.: Biosynthetic effects on the stable carbon isotopic compositions of algal lipids: Implications for deciphering the carbon isotopic biomarker record, Geochim. Cosmochim. Ac., 62, 1397-1406, 1998.

Schubert, C. J. and Calvert, S. E.: Nitrogen and carbon isotopic composition of marine and terrestrial organic matter in Arctic Ocean sediments: implications for nutrient utilization and organic matter composition, Deep Sea Res. I, 48, 789-810, 2001.

Simoneit, B. R. T.: Diterpenoid compounds and other lipids in deepsea sediments and their geochemical significance, Geochim. Cosmochim. Ac., 41, 463-476, 1977.

Simoneit, B. R. T.: Some Applications of Computerized GC-MS to the Determination of Biogenic and Anthropogenic Organic Matter in the Environment, Int. J. Environ. An. Ch., 12, 177-193, 1982.

Simoneit, B. R. T., Cardoso, J. N., and Robinson, N.: An assessment of the origin and composition of higher molecular weight organic matter in aerosols over Amazonia, Chemosphere, 21, 1285-1301, 1990.

Solomon, S., Mudie, P. J., Cranston, R., Hamilton, T., Thibaudeau, S. A., and Collins, E. S.: Characterisation of marine and lacustrine sediments in a drowned thermokarst embayment, Richards Island, Beaufort Sea, Canada, Int. J. Earth Sci., 89, 503-521, doi:10.1007/s005310000126, 2000.

Stein, R. and Macdonald, R. W.: The Organic Carbon Cycle in the Arctic Ocean, Springer, Berlin, Heidelberg, 2004.

Thoumelin, G., Bodineau, L., and Wartel, M.: Origin and transport of organic matter across the Seine estuary: Fatty acid and sterol variations, Mar. Chem., 58, 59-71, 1997.

Tolosa, I. and de Mora, S.: Isolation of neutral and acidic lipid biomarker classes for compound-specific-carbon isotope analysis by means of solvent extraction and normal-phase highperformance liquid chromatography, J. Chromatogr. A, 1045, 71-84, 2004a.

Tolosa, I., Vescovali, I., LeBlond, N., Marty, J.-C., de Mora, S., and Prieur, L.: Distribution of pigments and fatty acid biomarkers in particulate matter from the frontal structure of the Alboran Sea (SW Mediterranean Sea), Mar. Chem., 88, 103-125, 2004b

Tremblay, J.-É., Michel, C., Hobson, K. A., Gosselin, M., and Price, N. M.: Title Bloom dynamics in early opening waters of the Arctic Ocean. Limnol. Oceanogr., 51, 900-912, 2006.

Tremblay, J. É., Bélanger, S., Barber, D. G., Asplin, M., Martin, J., Darnis, G., Fortier, L., Gratton, Y., Link, H., Archambault, P., Sallon, A., Michel, C., Williams, W. J., Philippe, B., and Gosselin, M.: Climate forcing multiplies biological productivity in the coastal Arctic Ocean, Geophys. Res. Lett., 38, L18604, doi:10.1029/2011g1048825, 2011.
Volkman, J. K.: A review of sterol markers for marine and terrigenous organic matter, Org. Geochem., 9, 83-99, 1986.

Volkman, J. K.: Sterols in microorganisms, Appl. Microbiol. Biot., 60, 495-506, 2003.

Volkman, J. K., Johns, R. B., Gillan, F. T., Perry, G. J., and Bavor Jr., H. J.: Microbial lipids of an intertidal sediment- I. Fatty acids and hydrocarbons, Geochim. Cosmochim. Ac., 44, 1133-1143, 1980.

Volkman, J. K., Gllan, F. T., Johns, R. B., and Eglinton, G.: Sources of neutral lipids in a temperate intertidal sediment, Geochim. Cosmochim. Ac., 45, 1817-1828, 1981.

Volkman, J. K., Holdsworth, D. G., Neill, G. P., and Bavor Jr., H. J.: Identification of natural, anthropogenic and petroleum hydrocarbons in aquatic sediments, Sci. Total Environ., 112, 203-219, 1992.

Volkman, J. K., Barrett, S. M., Dunstan, G. A., and Jeffrey, S. W.: Sterol biomarkers for microalgae from the green algal class Prasinophyceae, Org. Geochem., 21, 1211-1218, 1994.

Volkman, J. K., Barrett, S. M., Blackburn, S. I., Mansour, M. P., Sikes, E. L., and Gelin, F.: Microalgal biomarkers: A review of recent research developments, Org. Geochem., 29, 1163-1179, 1998.

Vonk, J. E., van Dongen, B. E., and Gustafsson, Ö.: Lipid biomarker investigation of the origin and diagenetic state of sub-arctic terrestrial organic matter presently exported into the northern Bothnian Bay, Mar. Chem., 112, 1-10, 2008.

Wakeham, S. G., Hedges, J. I., Lee, C., Peterson, M. L., and Hernes, P. J.: Compositions and transport of lipid biomarkers through the water column and surficial sediments of the equatorial Pacific Ocean, Deep Sea Res. II, 44, 2131-2162, 1997.

Wang, Z. and Fingas, M. F.: Development of oil hydrocarbon fingerprinting and identification techniques, Mar. Pollut. Bull., 47, 423-452, 2003.

Yunker, M. B., Macdonald, R. W., Veltkamp, D. J., and Cretney, W. J.: Terrestrial and marine biomarkers in a seasonally ice-covered Arctic estuary - integration of multivariate and biomarker approaches, Mar. Chem., 49, 1-50, 1995.

Yunker, M. B., Backus, S. M., Graf Pannatier, E., Jeffries, D. S., and Macdonald, R. W.: Sources and Significance of Alkane and PAH Hydrocarbons in Canadian Arctic Rivers, Estuarine, Coast. Shelf Sci., 55, 1-31, 2002.

Yunker, M. B., Belicka, L. L., Harvey, H. R., and Macdonald, R. $\mathrm{W}$.: Tracing the inputs and fate of marine and terrigenous organic matter in Arctic Ocean sediments: A multivariate analysis of lipid biomarkers, Deep Sea Res. II, 52, 3478-3508, 2005.

Yunker, M. B., Macdonald, R. W., Snowdon, L. R., and Fowler, B. R.: Alkane and PAH biomarkers as tracers of terrigenous organic carbon in Arctic Ocean sediments, Org. Geochem., 42, 1109 $1146,2011$. 